University of New Mexico

UNM Digital Repository

Native Health Database Full Text

Health Sciences Center Archives and Special

Collections

1998

\title{
Cervical cancer screening among low-income women: results of a national screening program, 1991-1995.
}

HW Lawson

NC. Lee

SF. Thames

R. Henson

DS. Miller

Follow this and additional works at: https://digitalrepository.unm.edu/nhd

\section{Recommended Citation}

Lawson HW. Lee NC. Thames SF. Henson R. Miller DS. Cervical cancer screening among low-income women: results of a national screening program, 1991-1995. Obstetrics \& Gynecology 92(5):745-52. 1998

This Article is brought to you for free and open access by the Health Sciences Center Archives and Special Collections at UNM Digital Repository. It has been accepted for inclusion in Native Health Database Full Text by an authorized administrator of UNM Digital Repository. For more information, please contact disc@unm.edu. 
M

$\mathbf{N}$

o

G

$\mathbf{R}$

A

$\mathbf{P}$

H

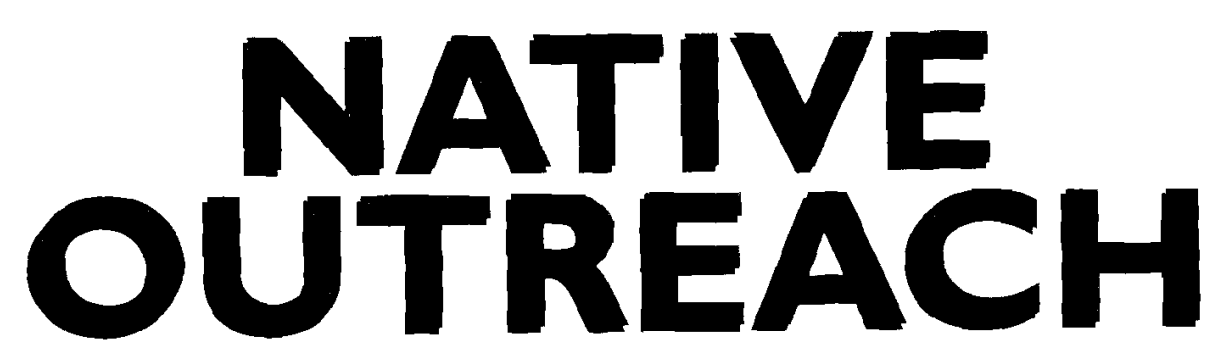

\title{
A Report to American Indian, Alaska Native, and Native Hawaiian Communities
}

\author{
NATIONAL INSTITUTES OF HEALTH \\ NATIONAL CANCER INSTITUTE
}




\section{Foreword}

\section{T} he National Cancer Institute (NCI) is pleased to present Native Outreach: A Report to American Indian, Alaska Native, and Native Hawaiian Communities - a monograph written specifically for community leaders, health professionals, and lay health workers serving those communities. Native Americans have higher mortality rates than the general U.S. population for several major cancer sites. Native Outreach focuses on the need to reduce those rates through effective interventions at the grassroots level.

NCI's mission, to conduct a comprehensive program of research to discover ways to reduce the burden of cancer, drives one of its primary goals--to translate tested methods for cancer prevention and control into tangible benefits for American communities. In the NCI Division of Cancer Control and Population Sciences, culturally sensitive methods for cancer prevention and control are being developed and tested for special populations. Through this monograph, we disseminate information arising from NCI research focused on Native Americans and their cancer control needs.

Today, we know that cancer is a group of diseases that results from a series of changes in genes that control cell growth and behavior. These genetic changes transform healthy cells into cancerous cells. The relationship of such genetic changes to the environment and to behaviors such as smoking and consumption of a diet high in fat is particularly relevant to cancer prevention. Environmental risks associated with smoking, diet, the community, workplace, and so on, probably account for more than 50 percent of all cancer cases. Smoking is the most preventable cause of death in the United States. Tobacco use is responsible for almost one in five deaths and accounts for nearly 30 percent of all cancer deaths in the United States. Similarly, scientists suggest that about one-third of U.S. cancer deaths each year are attributable to dietary factors. From another perspective on cancer control, we know that early detection of some cancers, such as those of the breast and cervix, can significantly improve cancer survival and reduce cancer mortality.

The Native Outreach monograph summarizes interventions designed to reduce cancer rates through studies focusing on breast and cervical cancers, education and screening, tobacco education and policy, smoking cessation and prevention, and nutrition education for Native Americans. In this volume, we document the planning, development, implementation, and evaluation of seven studies carried out between 1989 to 1996 from two of our Native American research programs: Avoidable Mortality From Cancer in Native American Populations and Primary Prevention of Cancer in Native American Populations. These studies identified key factors contributing to avoidable mortality from breast and cervical cancers, implemented programs to increase screening for these cancers, and focused on innovative interventions to eliminate or reduce cancer risks from tobacco and diet.

We encourage readers to use this volume as a guide for designing culture-appropriate, communitylevel activities. Whatever your needs are, we fervently hope that Native Outreach will provide adequate assistance and that your cancer control programs will be effective, ultimately reducing cancer's toll in your community.

Richard D. Klausner, M.D. Director, National Cancer Institute 


\section{Preface}

$\mathrm{T}$

his publication, Native Outreach: A Report to American Indian, Alaska Native, and Native

Hawaian Communities, is significant as it provides needed information on program implementation and data to our Native people. Cancer is rapidly becoming a serious threat in our communities. It is necessary to become more familiar with this threat and its causes, consequences, and treatment in order for us to successfully combat this disease.

This publication contains information not only on the morbidity and mortality of cancers in Native communities but also on projects that are successful and culturally relevant to our communities. This is an important and valuable contribution to our Native people, as it provides information and models that can be referenced and replicated. This is a necessary step in addressing the health of our communities and in helping us set up our own programs.

This manuscript is the first of its kind. It is designed to provide essential information to the Native population regarding the problem of cancer in our communities and the identification of what works in cancer prevention and control. Thanks to each of the researchers involved in these projects for their dedication and hard work in cancer control efforts in our American Indian, Alaska Native, and Native Hawaiian communities.

Lorraine Edmo Executive Director National Indian Education Association 


\section{Acknowledgments}

T he editors wish to thank the authors of this monograph and the principal investigators and co-investigators of the Avoidable Mortality From Cancer in Native American Populations Program and the Primary Prevention of Cancer in Native American Populations Program for their support of and commitment to this monograph. We also thank Drs. Larri Fredericks (Athabascan) (Center for American Indian Research and Education [CAIRE]) and Noma Roberson (Roswell Park Memorial Cancer Institute), who served as special reviewers and assistant editors of the monograph. This monograph would not have been possible without their dedication and hard work. We thank the following reviewers of the monograph for their astute comments and suggestions: Beverly Rodriguez. (Pomo); Barry Miller and Drs. Otis Brawley, Nancy Breen, and Susan Devesa (National Cancer Institute [NCI]); June Strickland (Cherokee)
(University of Washington); and Anthony Garcia (Apache) (University of California at Berkeley). We are especially grateful to the staff of the Biometry and Applied Research branches of NCI for their statistical review of this monograph. We also thank Arun Nevader (CAIRE) and Frank Mahaney (NCI) for their editing assistance and Barbara Bonaparte (NCI) for her technical assistance. And finally, we thank William Lynn (NCI) and NCI's Office of Cancer Communication staff members for their assistance in the publication of this monograph; Marilyn Massey (MasiMax Resources, Inc.) for overseeing the publication process; Deborah Bruskin, James Libbey, Theodora Radcliffe, Barbara Shine, Donna Cay Tharpe, Jacqueline Tressler, and Debra Waugh (R.O.W. Sciences, Inc.) for their assistance in copyediting and final preparation of this monograph; and Vernon Dunning and Jill Wagner (R.O.W. Sciences) for graphics and desktop publishing support. 


\section{The Editors}

Claudia Sanchez Glover, M.H.S., is a health statistician in the Office of Special Populations Research (OSPR) in the Office of the Director at the National Cancer Institute. She is the former program director for Native $\Lambda$ merican Cancer Control and director of the two programs discussed in this monograph, "Avoidable Mortality From Cancer in Native American Populations" and "Primary Prevention of Cancer in Native American Populations."
Felicia Schanche Hodge, Dr.P.H., is director of the Center for American Indian Research and Education (CAIRE) at the School of Public Health, University of California at Berkeley. Dr. Hodge is a Wailaki Indian from northern California and has extensive experience in formative research specializing in the development and evaluation of culturally sensitive cancer control intervention models in American Indian communities. She also teaches in the School of Social Welfare and is director of the American Indian Graduate Program, a recruitment/retention program at the University of California at Berkeley. 


\section{Table of Contents}

\section{Native Outreach: A Report to American Indian, Alaska Native, and Native Hawaiian Communities}

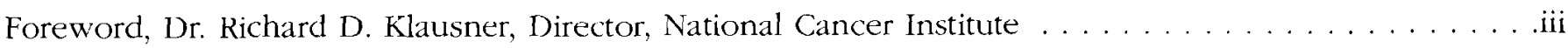

Preface, Lorraine Edmo, Executive Director, National Indian Education Association . . . . . . . . . . . v

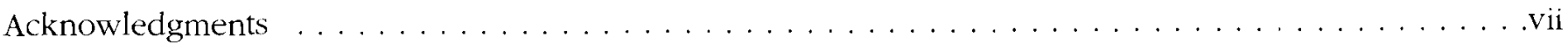

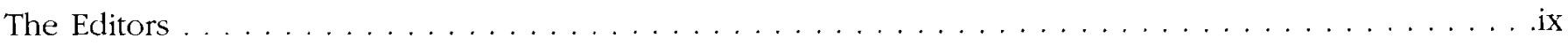

Chapter 1. The National Cancer Institute's Interventions in Native American Communities:

Background and Overview, Claudia Sanchez Glover, M.H.S., and Felicia Schanche Hodge, Dr.P.H. . . .1

Chapter 2. The Alaska Native Women's Health Project To Reduce Cervical Cancer,

Anne P. Lanier, M.D., M.P.H., Janet Kelly, M.S., M.P.H., and James Berner, M.D.

Chapter 3. Cervical Cancer Education for Native American Women in North Carolina:

The North Carolina Native American Cervical Cancer Prevention Project,

Ronny A. Bell, Ph.D., M.S., Mark B. Dignan, Ph.D., M.P.H., Robert Michielutte, Ph.D.,

Penny Sharp, Ed.1., Karen Blinson, and John G. Spangler, M.D., M.P.H. . . . . . . . . . . . 33

Chapter 4. Effects of a Culturally Tailored Intervention To Increase Breast and Cervical

Cancer Screening in Native Hawaiians, Richard Banner, M.D., Carolyn Cook Gotay, Ph.D.,

Rachelle Enos, M.P.H., Doris Segal Matsunaga, M.P.H., Nancy Hedlund, R.N., Ph.D.,

Brian F. Issell, M.D., and Ho'oipo DeCambra

Chapter 5. Enhancing Tobacco Control Policies in Northwest Indian Tribes,

Edward Lichtenstein, Ph.D., and Kerri Lopez

Chapter 6. "It's Your Life-It's Our Future" Stop Smoking Project,

Felicia Schanche Hodge, Dr.P.H., Larri Fredericks, Ph.D., and Patricia Kipnis, Ph.D. . . . . . . . . .67

Chapter 7. Pathways to Health: A Cancer Prevention Project for Native American Schoolchildren and Their Families, Sally M. Davis, Ph.D., Leslie Cunningham-Sabo, M.S., R.D., C.H.E.S., and Lori C. Lambert, M.A.

Chapter 8. Tobacco Use Prevention and Dietary Modification Among American Indian Youth in the Northeast, Steven Schinke, Ph.D., Kristin Cole, and Beverly Singer, Pl..D . . . . . . . . . . . . 93

Chapter 9. The National Cancer Institute's Research Efforts in Native American Communities: Approaches Used and Lessons Learned, Felicia Schanche Hodge, Dr.P.H., and Claudia Sanchez Glover, M.H.S. 
A. Glossary . . . . . . . . . . . . . . . . . . . . . . . . . . . . . . . . . . . 115

B. Bibliographies Associated with Studies . . . . . . . . . . . . . . . . . . . . . . . . . . . . 125

C. Highlights of Avoidable Mortality From Cancer in Native American Populations Program . . . . 129

D. Highlights of Primary Prevention of Cancer in Native American Populations Program . . . . . . 131

E. National Cancer Institute Research on Native Americans . . . . . . . . . . . . . . . . 135 


\title{
The National Cancer Institute's Interventions in Native American Communities: Background and Overview
}

\author{
Claudia Sanchez Glover and Felicia Schanche Hodge
}

\begin{abstract}
- Wis monograph presents the National Cancer Institute's (NCl) research efforts in Native American communities reported primarily to community leaders, health professionals, and lay health workers. As a result of targeted efforts, eight cooperative agreements were funded to design, develop, implement, and evaluate interventions in cancer prevention and control. These studies are historic because they represent the first concerted nationwide effort by $\mathrm{NCl}$ to address the cancer prevention and control needs of Native Americans. Although these studies were funded to enhance cancer prevention and control research and programs in Native American communities, they also can be used as valuable models for encouraging Native American community leaders, health professionals, and lay health workers to implement these types of studies in their native communities.
\end{abstract}

\section{INTRODUCTION}

For several years, NCI has taken steps to reduce the impact of cancer in Native American communities. In 1989, NCI invited investigators to apply for cooperative agreements to assess, with NCI's assistance, the effectiveness of cancer prevention and control intervention strategies in Native American populations, defined as American Indians, Alaska Natives, and Native Hawaiians. Two Requests for Applications (RFAs) were issued. Subsequently, NCI funded eight studies: four under the Avoidable Mortality From Cancer in Native American Populations RFA and four under the Primary Prevention of Cancer in Native American Populations RFA. These studies were conducted between 1989 and 1996. This monograph documents findings from seven of the eight studies. (For this monograph, references to all three Native American subpopulations are indicated by the term "Native Americans" unless otherwise noted.)

Although the goals of the avoidable mortality and primary prevention programs were similar in that they both addressed cancer prevention and control efforts in Native American communities, they differed in their approach. The Avoidable Mortality From Cancers in Native American Populations program addressed the effectiveness and efficacy of cancer prevention and control intervention strategies to increase appropriate use of screening procedures to reduce cancer rates and risks among Native Americans. Simply stated, these goals were to:

a Identify key factors that contribute to avoidable mortality from specific cancers, such as cervical and breast cancers; 
Increase appropriate use of screening procedures to reduce the rates of these cancers and/or risks; and

Evaluate the effectiveness of these interventions.

Avoidable mortality interventions are characterized by methods that help people overcome barriers to cancer prevention and control services. Typically, these barriers include behavioral/cultural barriers and health system/structural barriers. Behavioral/cultural barriers encompass language differences, psychosocial factors, cultural beliefs that may influence accessing cancer control services, and lack of knowledge and understanding of cancer prevention and control services. Health system/structural barriers include availability of cancer control services, financial limitations, and transportation problems.

The goals of the Primary Prevention of Cancer in Native American Populations program were to:

Develop innovative smoking and smokeless tobacco use prevention and cessation and/or dietary intervention programs; and

* Determine the long-term effectiveness of these programs.

Primary prevention is the complete avoidance of exposure to a carcinogen (1). Primary prevention also includes efforts to identify and modify behaviors and attitudes that have been recognized as risk factors for cancer. Primary prevention interventions are characterized by behavioral studies aimed at smoking prevention and cessation to prevent the onset of smoking behavior or to stop smoking behavior before it results in a person developing cancer. In addition, primary prevention interventions include nutrition studies that focus on modifying dietary behavior.

\section{PURPOSE OFTHE MONOGRAPH}

This monograph documents findings from the NCI's Native American cancer prevention and control research studies. It is designed and written primarily for Native American community leaders, health professionals, and lay health workers to promote cancer prevention and control activities at the community level. It also highlights the exciting collaborative efforts between American Indian, Alaska Native, and Native Hawaiian researchersas well as others who are experienced in Native
American research in cancer prevention and control-and NCI. This monograph's unique presentation includes research studies that employed a number of rescarch designs (i.e., randomized clinical trials, Solomon Four-Group Design (2), and pretest-posttest design) and intervention approaches (i.e., policy, educational, behavioral, and replication) to address cancer prevention and control programs for Native Americans.

Findings from the research studies are important because they increase our understanding of methods that may be successful in Native American communities. The methods discussed here are models for developing successful cancer prevention and control programs in Native American communitics. These studies have shown that conducting interventions in Native American communities requires the cooperation and involvement of the community in all phases of the health-promoting activity for it to be successful and culturally appropriate. In addition, this monograph documents the need for Native American communities to promote further investigation in the area of cancer prevention and control.

This monograph is organized into nine chapters: an introductory chapter, seven chapters that describe each of the studies in detail, and a concluding chapter. Three of the chapters describe the avoidable mortality studies, and four chapters describe the primary prevention studies, respectively. The final chapter of the monograph summarizes the studies and discusses the four research intervention approaches utilized by the research teams: policy change, behavior change, education, and replication. A policy is an overall plan that supports the general goals and acceptable proccdures of a governmental body. The implementation of a new or revised policy is often the most difficult, because designing and implementing policies is a lengthy procedure requiring collaboration. Behavior change influences an individual's or group's response or action. Education increases an individual's level of knowledge. Replication of proven intervention models is a more common approach to addressing community health needs. In many cases, using a previously tested intervention reduces the overall planning and development stages of the study. 


\section{NATIVE AMERICANS AND CANCER: THE NEED FOR SPECIAL STUDIES}

One of NCl's goals is to ensure that the burden of cancer is reduced in all communities, including Native American communities. The history of NCI reflects that commitment.

NCI is the premier cancer research agency of the Federal Government. Located within the National Institutes of Health, NCI is charged with conducting a comprehensive program of research to identify and promote ways to reduce the burden of cancer. To that end, NCI conducts research on cancer risk factors, advancement of treatment and diagnosis, and development of prevention and control methods. NCI seeks to design effective strategies for disseminating information on research results to the public and the Nation's health professionals.

The Office of Special Populations Research (OSPR) is located within the Office of the Director, NCI. OSPR was established in June 1996 to advise and guide the Director of NCI on policy, programs, research, and issues pertaining to special populations. The reason for creating this Office was to ensure that the cancer problem in special populations (e.g., minorities, underserved individuals, and elderly persons) is adequately addressed. The Office also provides continued leadership at the Federal Government level in addressing cancer rescarch, programmatic, and outreach needs for special populations. Thus, OSPR serves as a focal point to provide leadership and coordination of research related to minorities and special populations.

In 1981, NCI set forth principles for cancer control research on targeted populations. In 1985, the Secretary of Health and Human Services' Task Force on Black and Minority Health strongly encouraged research on the effectiveness of strategies for health system intervention and preventive services in minority communities (3). In 1986, NCI created the Special Populations Studies Branch (SPSB) within the Cancer Control Science Program (now called the Division of Cancer Control and Population Sciences), with the charge to reduce and eliminate differences in cancer incidence, mortality, and survival between minority and special populations and the general population. In 1989, the two initiatives (RFAs) that resulted in the funding of the avoidable mortality and primary prevention studies were introduced by the SPSB staff.

\section{GOALS AND OBJECTIVES OF THE NATIVE AMERICAN STUDIES}

\section{Avoidable Mortality From Cancer in Native American Populations}

\section{Prevention of Cervical Cancer in Alaska Native Women}

The goal of the Alaska Native women's study was to reduce morbidity and mortality from cervical cancer among Alaska Native women age 20 and older. The project objectives were to (1) assess knowledge and attitudes about cervical cancer, its causes, early detection, and treatment; (2) determine the frequency (prevalence) of Pap testing; (3) assess attitudes and satisfaction regarding cancer prevention services, particularly the Pap test; (4) identify barriers to preventive health care; (5) increase the availability of women's health services, particularly services offered by female practitioners; (6) improve the followup of women with abnormal Pap tests; and (7) develop culturally appropriate educational materials. In this monograph, this project is also referred to as the "Women's Health Project."

\section{North Carolina Native American Cervical Cancer Prevention Project}

The North Carolina Native American women study's goal was to reduce cervical cancer mortality among American Indians. The primary objective was to increase the proportion of women age 18 and older who received Pap smears at appropriate intervals and who returned for followup care when necessary. The study was implemented in two North Carolina populations of American Indians, the Eastern Band of the Cherokee and the Lumbee.

\section{The Wai anae Coast Cancer Research Project}

The goal of the Wai anae study was to reduce the disproportionate cancer mortality from breast and cervical cancers among Native Hawaiian women. The study objectives were to (1) test the effectiveness of a culturally sensitive intervention as a means of increasing breast and cervical cancer screening practices; and (2) increase knowledge, attitudes, and behavior scores among Native Hawaiian women. This project targeted women 18 years of age and older. 


\section{Primary Prevention of Cancer in Native American Populations}

\section{Tobacco Policy Intervention in Nortbwest Indian Tribes}

The goal of the tobacco policy study was to change tribal policies so as to alter individual smoking behavior and to help protect individuals from exposure to tobacco smoke. The objectives were to (1) develop a consultative process that assisted Indian tribes in creating and implementing more explicit, comprehensive, and stringent tobacco use policies; (2) evaluate the process by means of a prospective randomized design that assigned tribes to immediate or clelayed intervention; (3) assess the impact of policy intervention on smoking knowledge, norms, attitudes, intentions, and behavior among influential tribal leaders; (4) evaluate the relationship of tobacco use policies and level of antitobacco behavior; and (5) develop a manual and set of guidelines for tobacco policy councils and organizations.

\section{Smoking Cessation for American Indians}

The smoking cessation study's goal was to increase long-term smoking cessation among American Indian populations in northern California through a reproducible clinic-based program. The objectives were to (1) identify needs and barriers related to smoking cessation; (2) estimate the prevalence of smoking and smokeless tobacco use and the correlates of those behaviors among American Indian clinic users in northern California; (3) modify the previously tested, physician-initiated self-help snoking cessation model for the American Indian health clinic setting; and (4) evaluate the effectiveness of the intervention. This study targeted American Indians age 18 and older. In this monograph. this study is also reforred to as the "It's Your Life-It's Our Future Smoking Cessation Project."

\section{Soutbwestern Cancer Prevention Project for American Indians}

The Pathways to Health study's goal was to promote healthy lifestyle behaviors among fifth- and seventhgrade students from several Indian tribes in New Mexico. The primary objective was to develop, implement, and evaluate a multidisciplinary program designed to promote the avoidance of cigarette smoking and smokeless tobacco use and to encourage consumption of a low-fat, high-fiber diet for students and their families. In this monograph, this study is also referred to as the "Pathways to Health Project."

\section{Reducing Cancer Risks Among Native American Adolescents}

The goal of the Native American adolescents study was to advance the science of cancer risk reduction interventions for younger American Indians. The primary objective was to study the efficacy of an intervention to prevent tobacco use and modify dietary habits among American Indian youth in seven northeastern states.

\section{HIGHLIGHTS OF RESULTS FROM THE NATIVE AMERICAN STUDIES}

Native Americans face a significant gap between cancer prevention and control necds and existing services. The NCI-funded studies represent a major step toward closing that gap

As shown in Figure 1, the studies, although concentrated mainly in the western part of the United States-Hawaii, Alaska, Oregon, New Mexico, and California-were also located in the Northeast and Southeast, New York, and North Carolina, respectively. Thus, a wide spectrum of Native Americans, including many Indian tribes, Native Hawaiians, and Alaska Natives, benefited from these studies in their respective communities. The results from these studies can be adapted for other Native Americans in other States and regions. Each chapter in this monograph contains sufficient details about how these studies were conducted so they may be replicated in other Native American communities.

It should be noted that the studies varied in terms of study topic, approach, site, and methodology. Some involved methods to increase breast and cervical cancer screenings; others involved interventions to prevent the onset of smoking behavior and smoking cessation programs; still others were school-based interventions to prevent and control tobacco use and modify diets; and one intervention changed tribal smoking policies. The settings for these studies were communities, tribes, clinics, and schools.

The research design used by most of the research teams was a pretest-posttest design. Although this design is useful for determining some change as a 


\section{FIGURE I: Location of NCI-Supported Native American Studies}

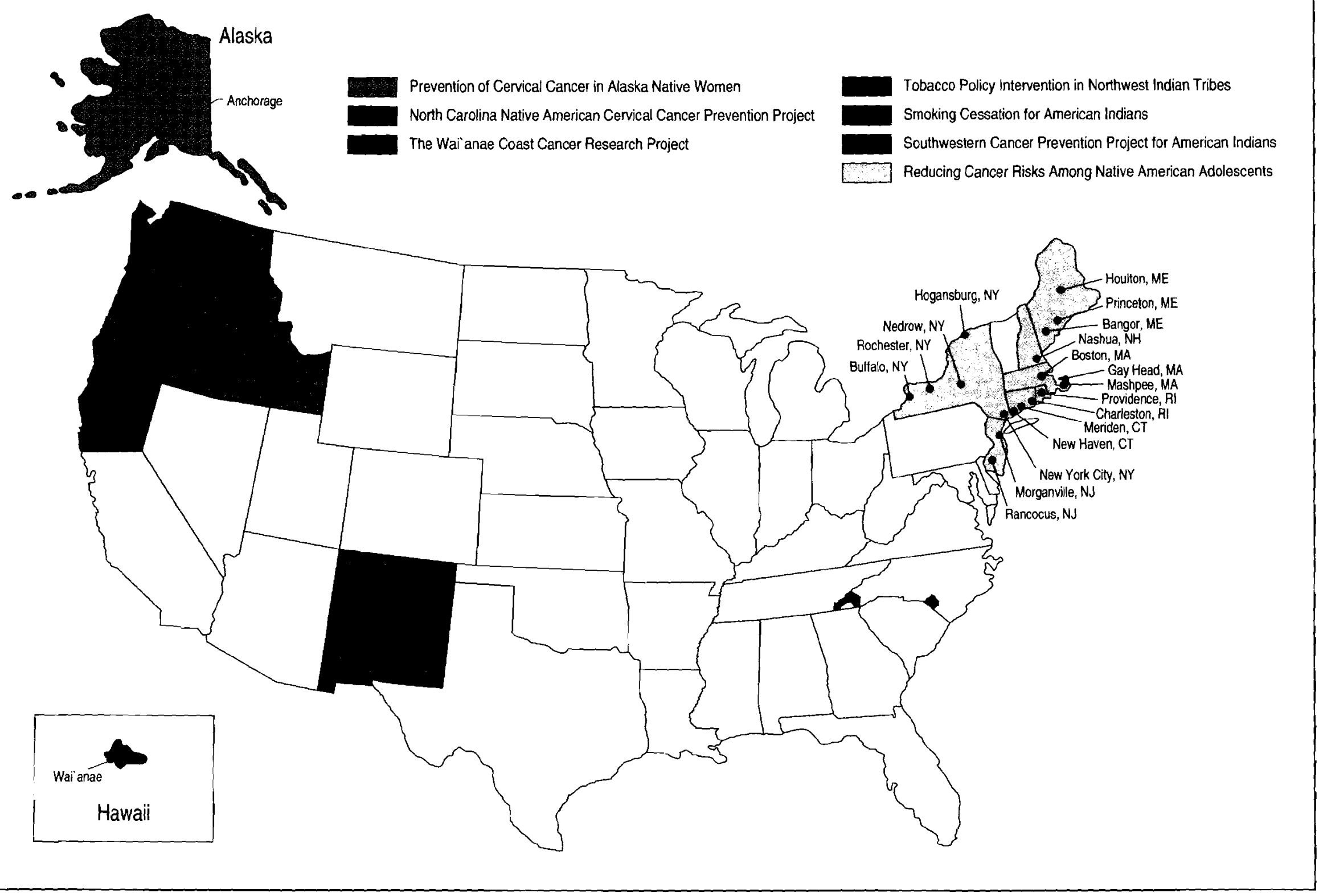


result of an intervention, it is not as robust as an experimental design that uses an experimental and a control group to determine the impact of an intervention on knowledge, attitudes, and behavior.

These studies received widespread support among Native American people because Native Americans were involved in every phase of the studies. Culturally sensitive approaches and materials were used (as shown on the following pages), and each study included Native Americans on the research team. Native Americans served as advisers to the studies, and community leaders were also consulted. Several of the studies (e.g., those in Hawaii, California, New Mexico, North Carolina, and Alaska) used focus groups to help plan, develop, and implement the interventions.

For example, the Hawaii study's use of focus groups resulted in use of the kokua group intervention because the focus group believed that method would be most effective in their community. Kokua is defined as a mutual willingness to help others without an expectation of return. The kokua intervention, delivered by trained Native Hawaiian health educators in a group setting, consisted of education to increase breast and cervical cancer screenings in the Native Hawaiian community. After the kokua group intervention, study participants showed positive gains in knowledge, and a significant number of women at posttest indicated that they were planning to get a Pap test and mammogram.

Focus groups also helped the California smoking cessation study increase its cultural sensitivity to the Native American community. Focus group sessions were conducted to determine beliefs, barriers, and smoking cessation methods that would be acceptable in these communities. Results from the study show that 30 percent of smokers at clinics promoting the smoking cessation program attempted to quit smoking during the study, in contrast to 20 percent of those at clinics providing standard care.

During the 2 years that its curriculum (intervention) was being developed, pilot-tested, and revised, the New Mexico study team held separate focus groups with American Indian teachers, parents, and school administrators from participating schools to elicit their advice on key cultural concepts and appropriate methods for use in their schools and communities. One finding of the study was that seventh-grade intervention students reported higher levels of cigarette use at posttest than at pretest. The research team surmised that this may have been because seventh graders participated in ceremonial activities that included traditional tobacco use. These results indicate that the sixth grade may be a critical transitioning period for American Indian students in regard to behavioral intentions to begin tobacco use.

An important lesson learned from the New York study, which also focused on American Indian youth, was that an integrated approach, utilizing both tobacco use prevention and dietary modification, promoted better outcomes than an isolated approach. The target population for this studyfourth, fifth, sixth, and seventh graders--was not a captive audience of classroom students as were students in the New Mexico study. The New York youths were recruited from the community.

Both the Hawaii and North Carolina studies used Native American lay health educators to deliver their interventions. The North Carolina intervention was delivered to women during two personal visits to their homes. Results from the North Carolina study indicated that the Cherokee women had increased knowledge after the intervention and were more likely than women in the control group to report obtaining a Pap smear during the preceding year. The Lumbee women also had increased knowledge and behavioral intentions but were less likely to obtain Pap smears than the control group.

The Tobacco Policy Intervention in Northwest Indian Tribes study (Washington, Oregon, and Idaho) used a unique cancer prevention and control method. The approach relied heavily on the structure and political independence of Indian tribes, which the research team thought was applicable to reducing other health risk factors. The changes resulted in stronger smoking policies, and the number of tribes with smoke-free policies doubled during the intervention.

Finally, the Alaska study documented the lack of accurate Pap test data for Native women. Before the study was implemented, there were few data on how frequently Native women received a Pap test and on the knowledge, attitudes, and behavior of Alaska Native women with respect to cervical cancer. The Alaska study addressed this problem. Results from the study show that among participants in the Alaska study, the percentage of women who 


\section{Prevention of Cervical Cancer in \\ Alaska Native Women}

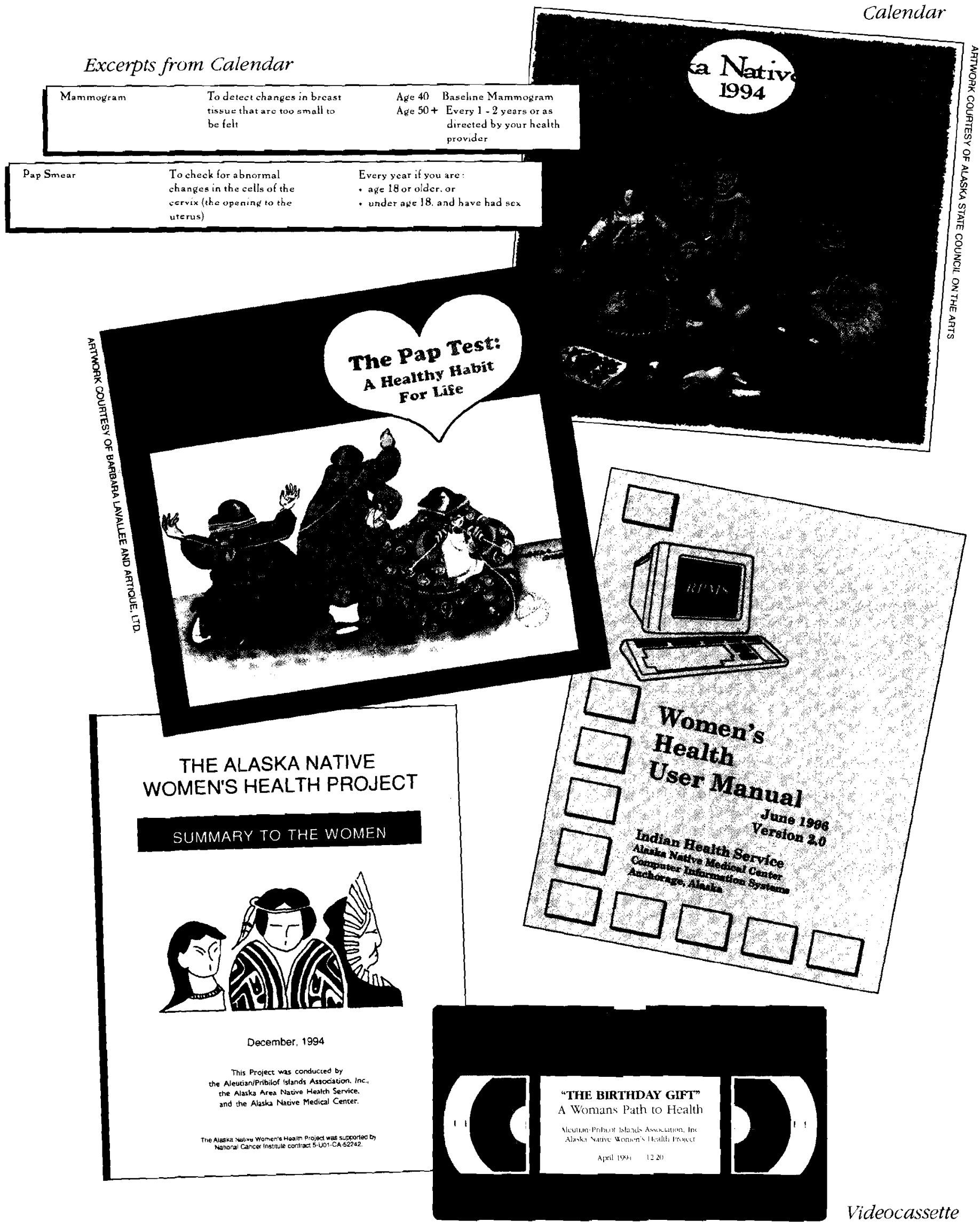




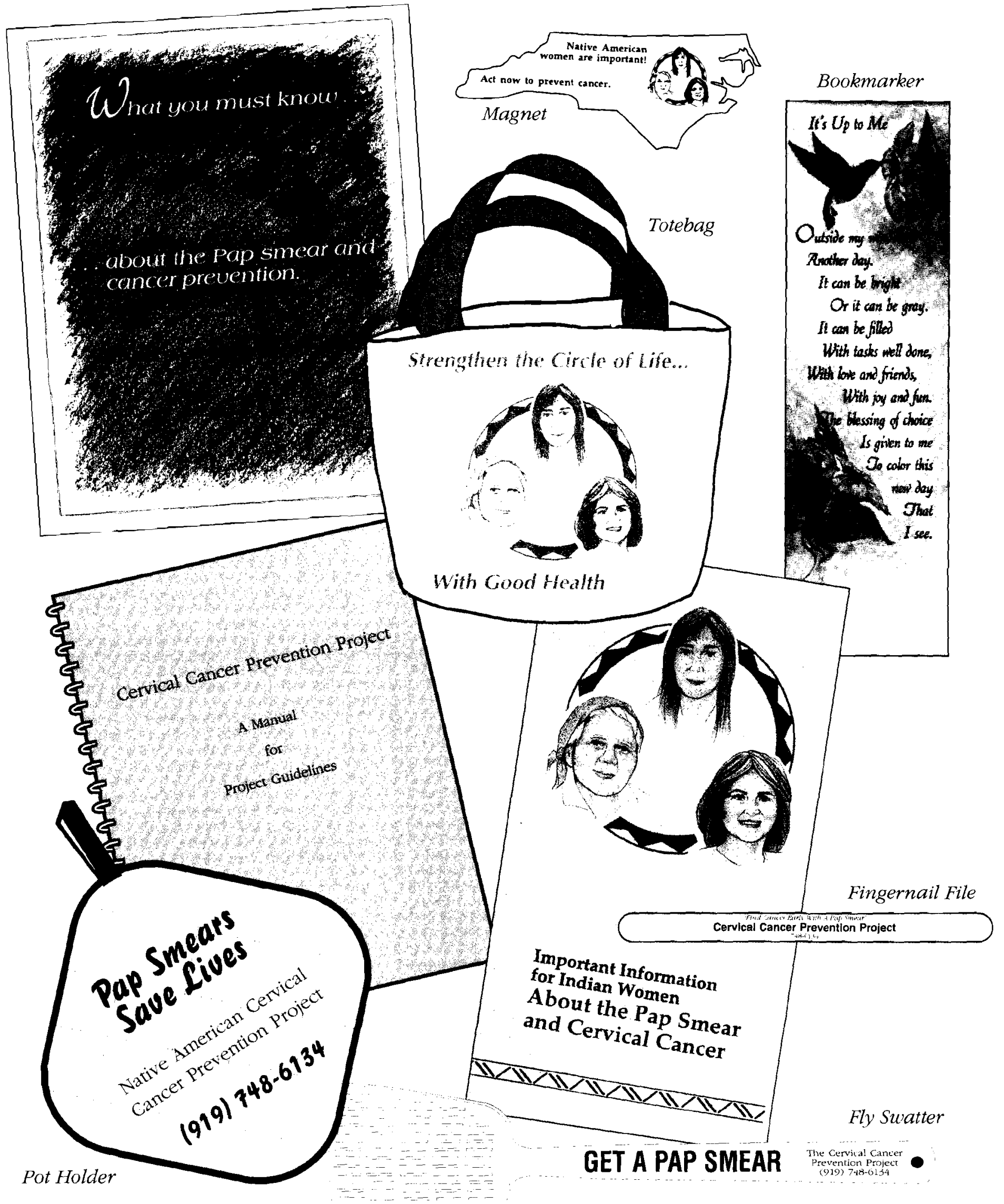




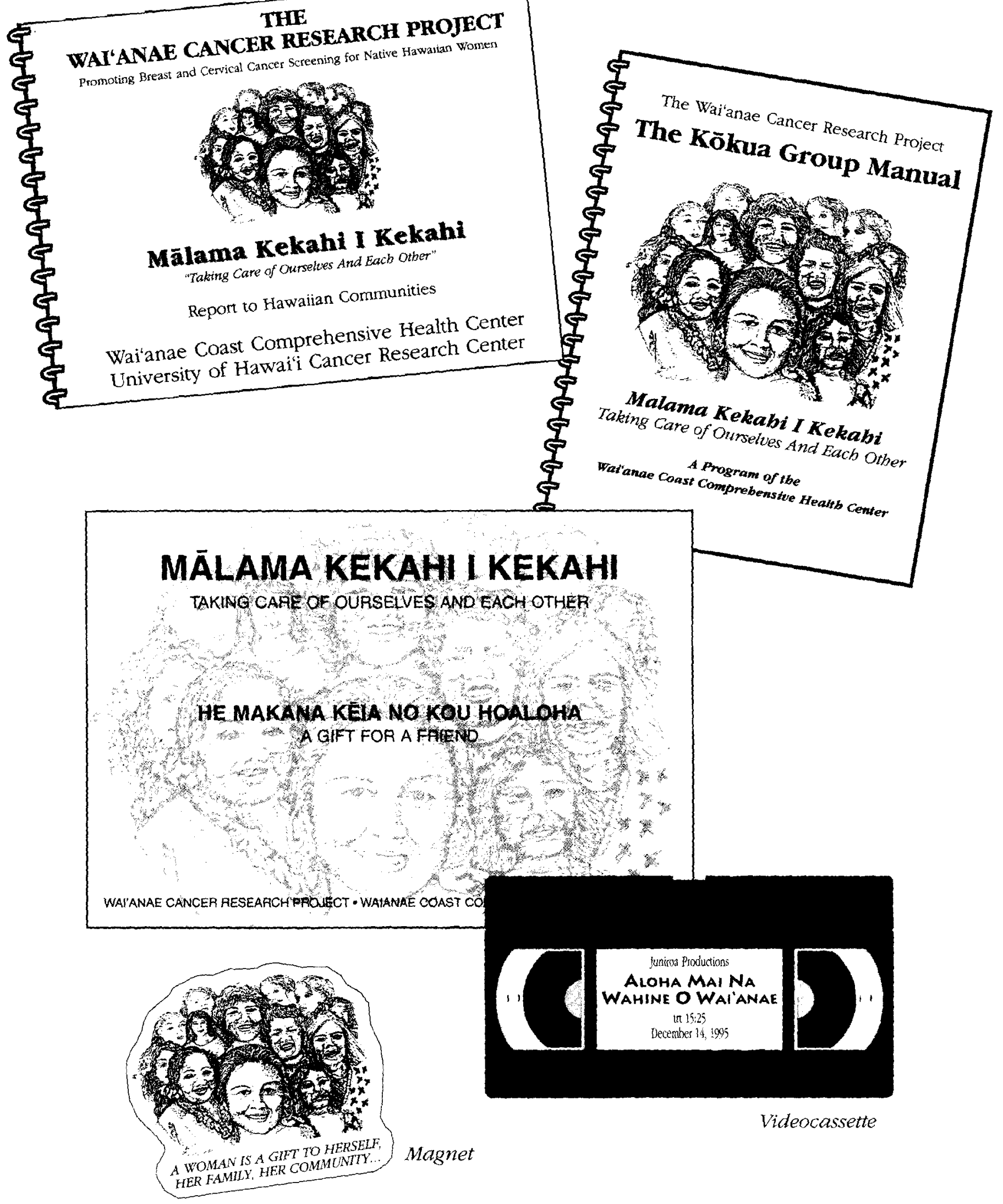




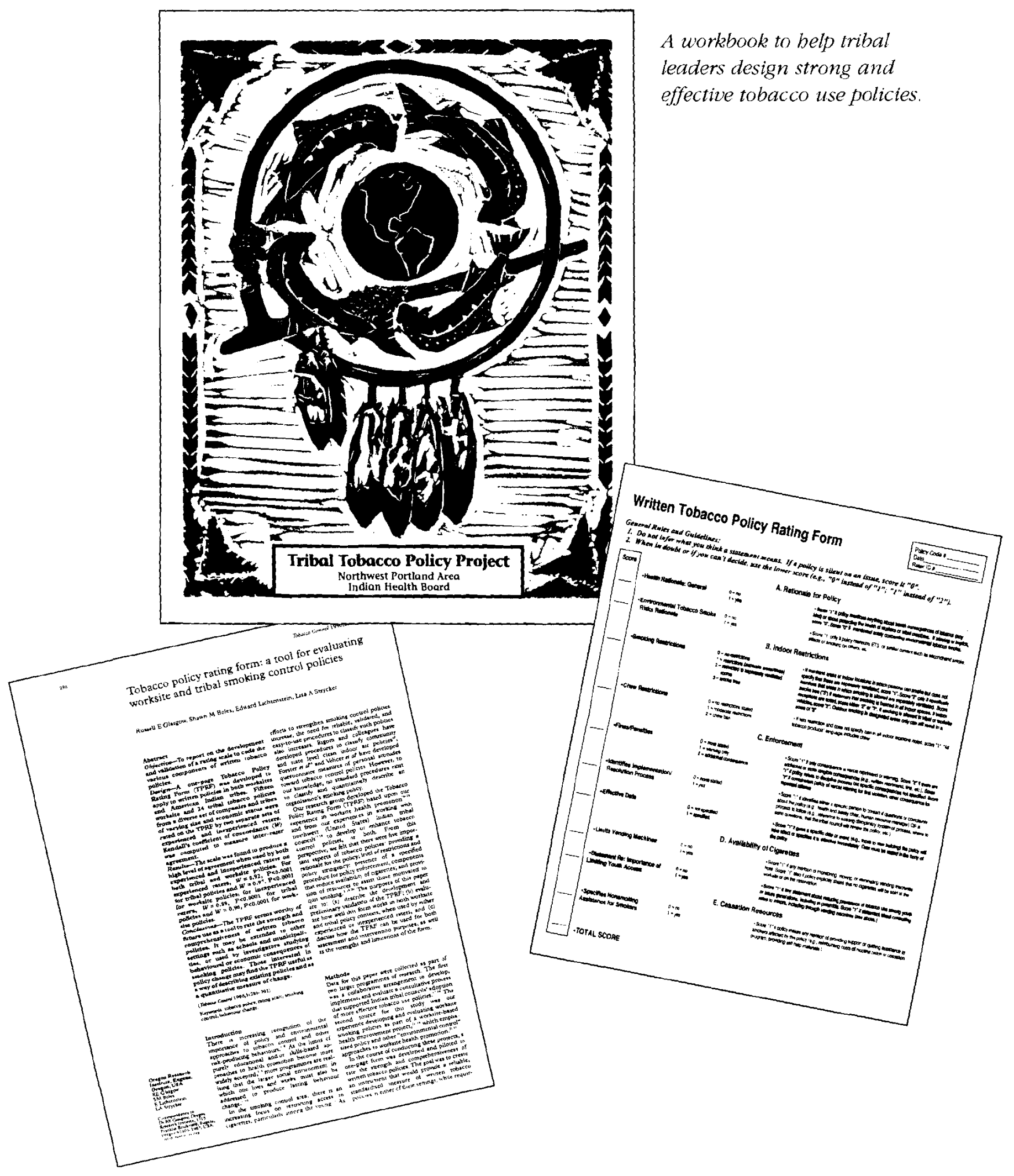




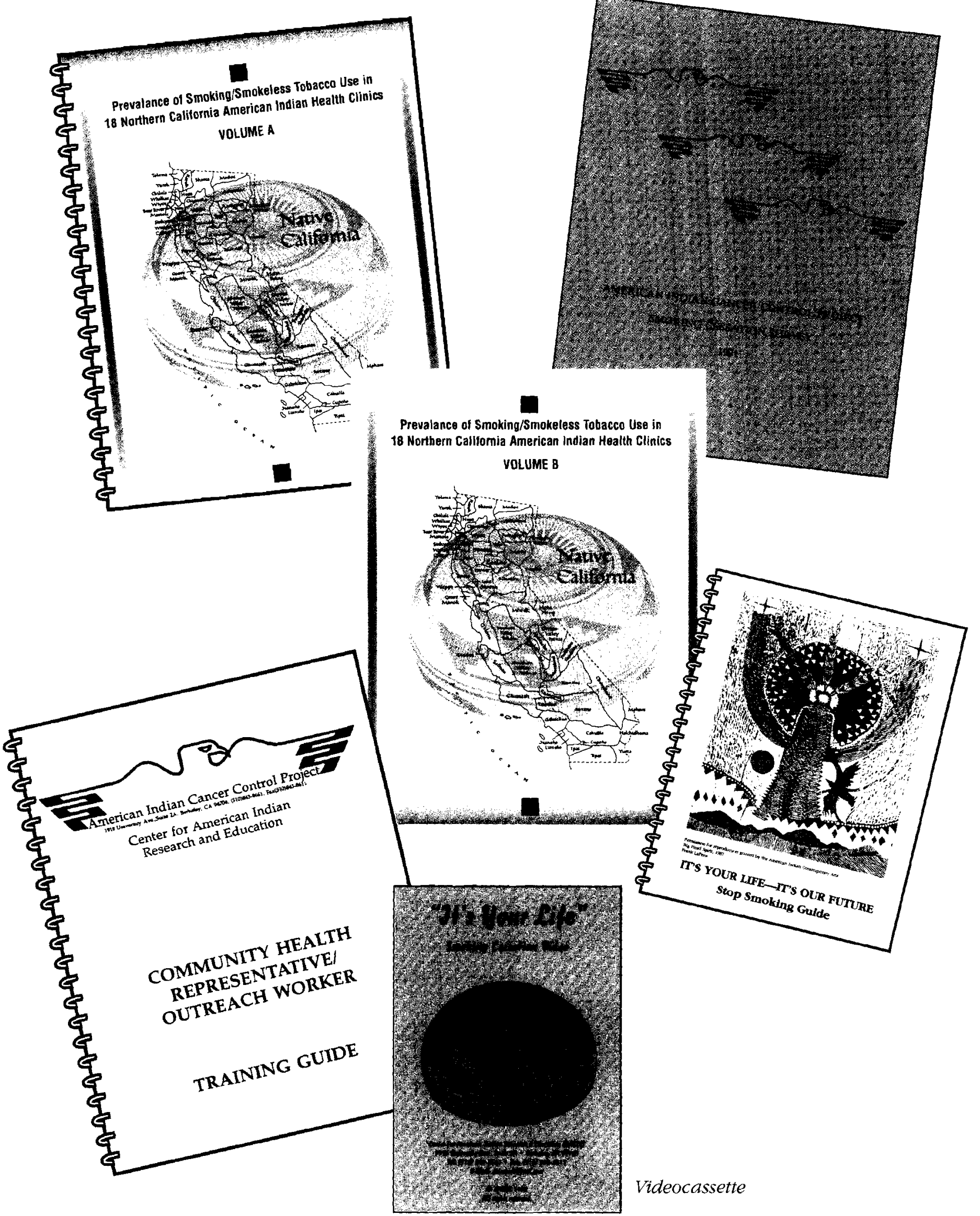




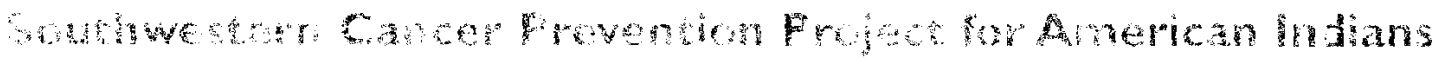

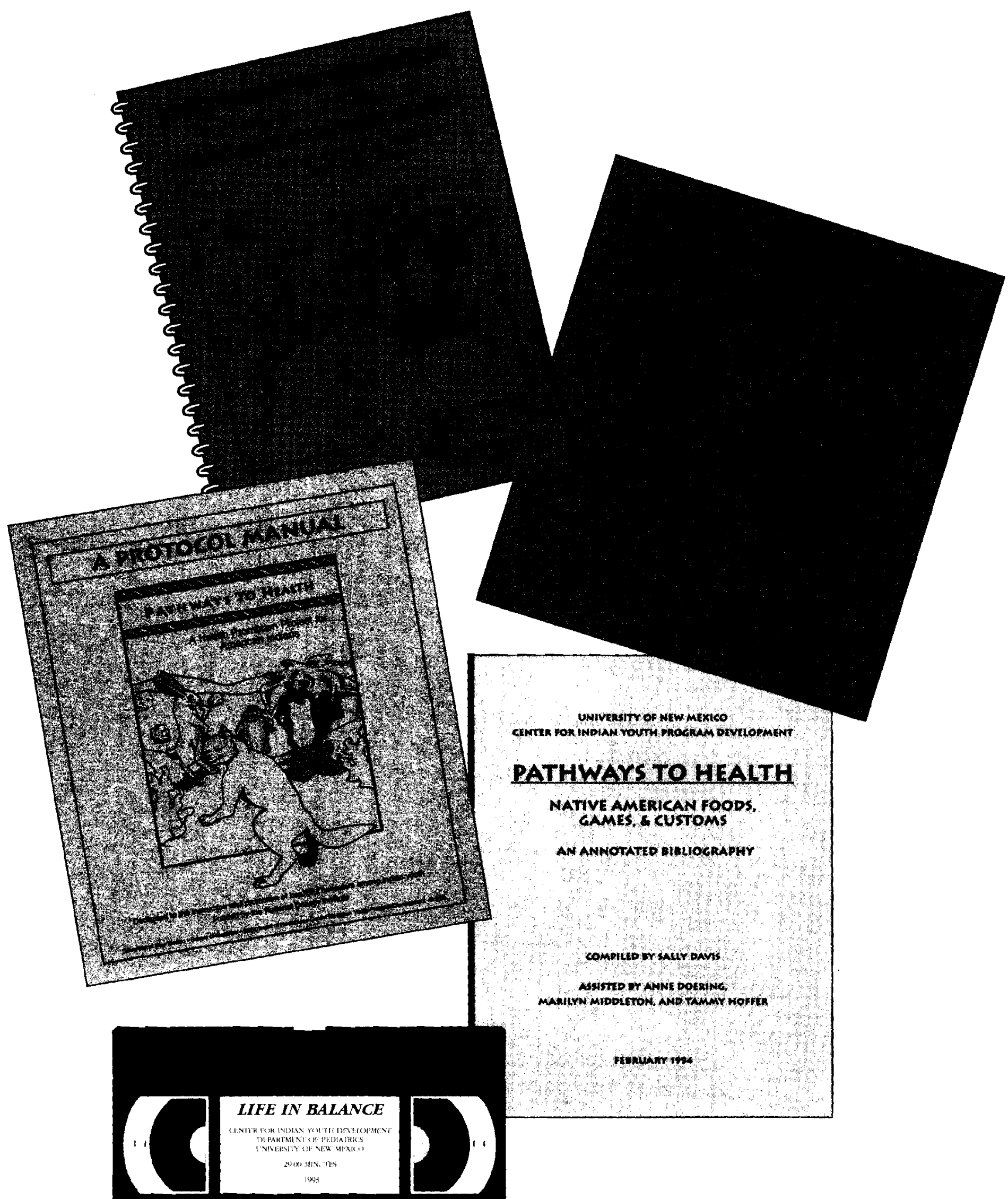

Videocassette 
Reducing Cancer Risks Among

Native American Adolescents

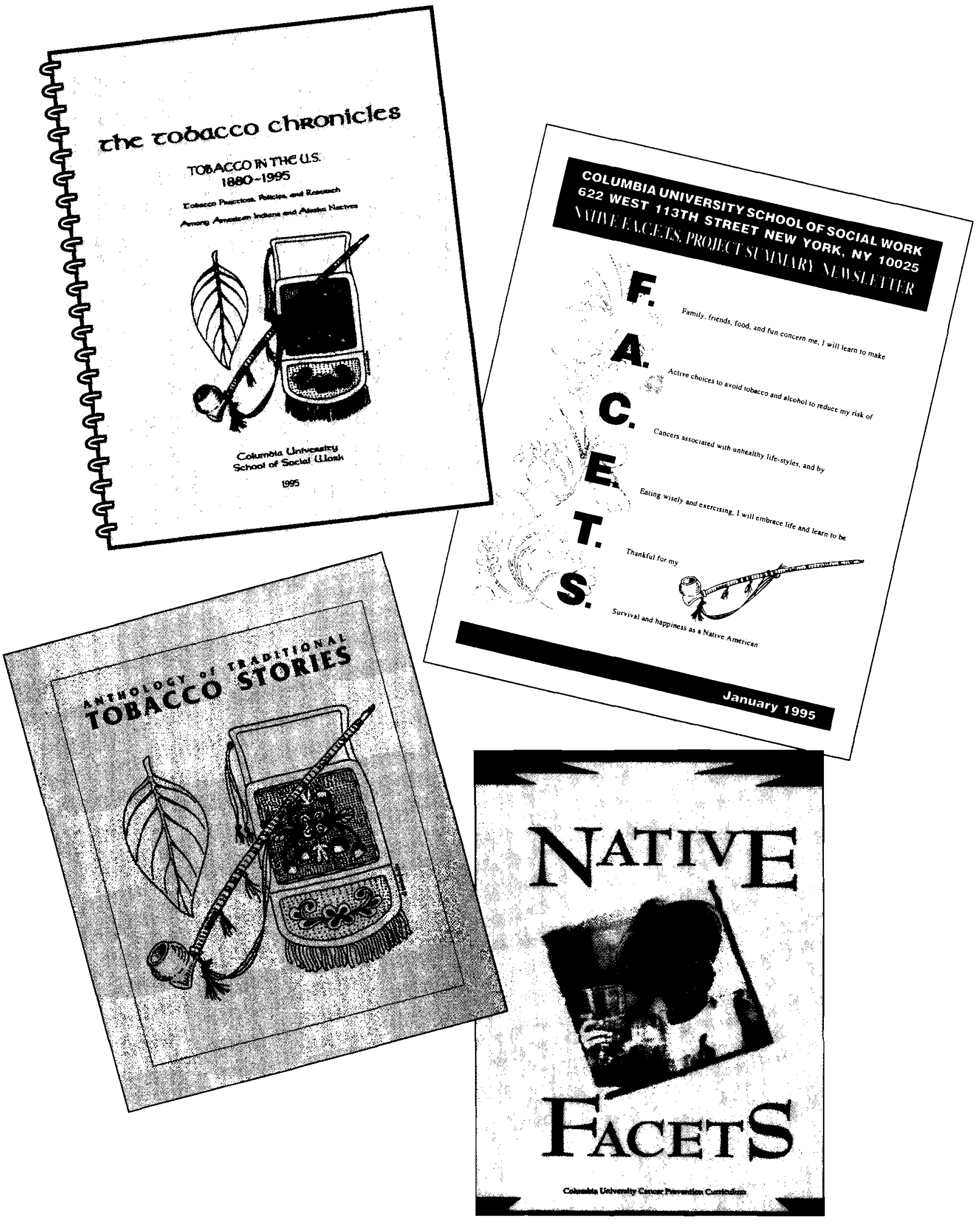


had not had a Pap test declined from 48 percent to 20 percent. In contrast, there was little improvement in screening among the control group (i.e., those who had not participated in the study).

\section{CANCER RISK FACTORS AMONG NATIVEAMERICANS}

Native Americans live in a complicated and changing cultural and social environment, which includes repeated exposure to risk factors related to behavior-based health problems. These risk factors include tobacco abuse and poor nutrition (4).

Significant changes in lifestyles and the adoption of "Western" habits related to nutrition have contributed to poor health among Native Americans. Native diets are predominately high in saturated fats and sugars and low in fiber. Obesity is a serious problem leading to diabetes and heart disease. American Indians nationwide are more overweight than the general population, with a rate of 33.7 percent for American Indian men (compared with 24.1 percent for the total U.S. male population) and 40.3 percent for American Indian women, which is significantly greater than the U.S. rate of 25.0 percent (5).

Historically, Native Americans combined a highfiber, low-fat diet with physical fitness and exercise. Today, lifestyles are sedentary and traditional foods have been replaced with high-fat and low-fiber foods. Lifestyle changes have increased Native Americans' cancer risk in recent years. Lifestyle factors contributing to poor health also include a diet high in salt, cigarette smoking, smokeless tobacco use, heavy alcohol consumption, obesity, and lack of exercise ( 6 ).

The rate of smoking for American Indians and Alaska Natives is reported to be among the highest of all racial groups in the United States. Recent survey data show higher smoking rates, more "heavy" smokers, and fewer never-smokers among American Indians/Alaska Natives than among other ethnic groups (7). According to aggregate data from the 1994 and 1995 National Health Interview Survey, 39.3 percent of American Indian and Alaska Native men and 32.9 percent of American Indian and Alaska Native women reported that they currently smoke (8).

The Indian Health Service reports that two out of every five deaths of American Indians in the United
States are attributed to smoking (9). Smoking increases rates of cardiovascular disease, cancer (including cancers of the lung, oral cavity, esophagus, bladder, kidney, pancreas, and cervix uteri), low birth weight, birth defects, and sudden infant death syndrome. Although lung cancer incidence rates have been reported to be low among American Indians as a group, there is wide variation by tribal affiliation and region. The 5-year relative survival rate for lung cancer is the lowest of any group (10).

According to some reports, smokeless tobacco ("chew," "snuff," "chaw," etc.) use among Alaska Native and American Indian youth begins before cigarette use. In fact, it appears that those at highest risk for early and frequent smokeless tobacco use are Native American youth of both sexes. Among Alaska Native and American Indian adolescents, 34 percent of all females and 42.6 percent of all males report weekly smokeless tobacco use (11).

There are reports that prevention measures, such as screening and early detection for breast cancer (mammography) and cervical cancer (Pap smear), are practiced less frequently by Native Americans (12). This may point to cultural, social, and financial barriers in need of special attention.

Before 1940, more than 90 percent of American Indians lived on reservations set aside by the Federal Government (13). Today, a large portion of American Indians reside in urban areas far from their ancestral homelands and extended families. Adaptation to new lifestyles adds stress for these relocated Indians.

Stressful cultural change can separate people from health-protective cultural practices and social support, placing them in more isolated circumstances. This is particularly threatening to minority populations that already have limited access to social resources. The stress, isolation, and limited access to social resources can heighten cancer risks in many ways. For example, high rates of tobacco and alcohol use and poor diets, high in fat and low in fiber, may be the most obvious result of such a lifestyle. However, less obvious, there also may be less knowledge about health problems and awareness of health services, attitudes, and behaviors that delay diagnosis and care. This may influence an individual's likelihood of taking action to protect personal health and the health of family members. 
Moreover, poverty remains widespread among Native Americans, and low socioeconomic status contributes to the patterns of disease (14). Native Americans are overrepresented in the lower socioeconomic class. The lack of financial resources affects quality, access, utilization, and delivery of health services, including cancer control services such as cancer screening. Low-income status is linked to edu- cational attainment, employment status, occupational category, tobacco use, and dietary practices, all of which are related to cancer risk profiles.

\section{EPIDEMIOLOGY OF CANCER AMONG NATIVE AMERICANS}

Cancer has become a significant health problem among Native Americans. This is reflected in cancer

\section{TABLE 1: SEER Incidence Rates, Men, 1988-1992}

\begin{tabular}{|c|c|c|c|c|c|c|}
\hline Cancer Site & $\begin{array}{l}\text { Alaska } \\
\text { Native }\end{array}$ & $\begin{array}{l}\text { American } \\
\text { Indian }\end{array}$ & Hawaiian & Black & White & Hispanic \\
\hline All Sites & 372.0 & 196.0 & 340.0 & 560.0 & 469.0 & 319.0 \\
\hline Brain \& ONS & $*$ & $*$ & $*$ & 4.5 & 7.8 & 5.2 \\
\hline Colon \& Rectum & 79.7 & 18.6 & 42.4 & 60.7 & 56.3 & 38.3 \\
\hline Esophagus & $*$ & $*$ & 9.4 & 15.0 & 5.4 & 4.4 \\
\hline Kaposi's Sarcoma & $*$ & $*$ & $*$ & 5.7 & 5.8 & 5.9 \\
\hline Kidney \& Renal Pelvis & $(19.0)^{* *}$ & 15.6 & 9.8 & 12.8 & 11.9 & 10.0 \\
\hline Larynx & * & $*$ & $*$ & 12.7 & 7.5 & 5.1 \\
\hline Leukemias & $*$ & $*$ & 10.8 & 11.5 & 13.5 & 9.4 \\
\hline Liver \& IBD & $*$ & $(13.1)^{* *}$ & $*$ & 6.9 & 3.7 & 6.7 \\
\hline Lung \& Bronchus & 81.1 & 14.4 & 89.0 & 117.0 & 76.0 & 41.8 \\
\hline Non-Hodgkin's Lymphoma & $*$ & * & 12.5 & 13.2 & 18.7 & 14.1 \\
\hline Hodgkin's Disease & * & * & $*$ & 2.3 & 3.3 & 2.5 \\
\hline Melanoma & * & $*$ & $*$ & 1.0 & 14.5 & 2.7 \\
\hline Multiple Myeloma & $*$ & * & * & 11.3 & 5.0 & 4.2 \\
\hline Nasopharynx & * & * & * & 1.0 & 0.6 & 0.6 \\
\hline Oral Cavity & $*$ & * & 11.7 & 20.4 & 14.6 & 8.9 \\
\hline Pancreas & $*$ & $*$ & 10.9 & 14.0 & 9.8 & 8.0 \\
\hline Prostate & 46.1 & 52.5 & 57.2 & 180.6 & 134.7 & 89.0 \\
\hline Stomach & 27.2 & $*$ & 20.5 & 17.9 & 10.2 & 15.3 \\
\hline Testis & $*$ & * & $*$ & 0.8 & 5.0 & 2.9 \\
\hline Thyroid & $*$ & $*$ & * & 1.4 & 2.6 & 2.0 \\
\hline Urinary Bladder & $*$ & $*$ & $*$ & 15.2 & 31.7 & 15.8 \\
\hline
\end{tabular}

Note: Rates are "average annual" per 100,000 population, age-adjusted to 1970 U.S. standard.

* = rate not calculated when feuer than 25 cases; data source for American Indians is New Mexico.

( )** = Rates based on fewer than 25 cases are included only for the top five most frequently diagnosed cancers for etbnic group. These rates may be subject to greater variability than other rates which are based on larger numbers. ONS = other nervous system; IBD = intrabepatic bile duct. SOurce: SEER Program, National Cancer Institute (see Glossary for additional information). Miller et al., 1996 (15). 


\section{TABLE I: SEER Incidence Rates, Women, $1988-1992$ (continued)}

\begin{tabular}{|c|c|c|c|c|c|c|}
\hline Cancer Site & $\begin{array}{l}\text { Alaska } \\
\text { Native }\end{array}$ & $\begin{array}{l}\text { American } \\
\text { Indian }\end{array}$ & Hawaiian & Black & White & IIispanic \\
\hline All Sites & 348.0 & 180.0 & 321.0 & 326.0 & 346.0 & 243.0 \\
\hline Brain \& ONS & $*$ & $*$ & $*$ & 3.4 & 5.4 & 3.8 \\
\hline Breast & 78.9 & 31.6 & 105.6 & 95.4 & 111.8 & 69.8 \\
\hline Cervix Uteri & 15.8 & 9.9 & 9.3 & 13.2 & 8.7 & 16.2 \\
\hline Colon \& Rectum & 67.4 & 15.3 & 30.5 & 45.5 & 38.3 & 24.7 \\
\hline Corpus Uteri & $*$ & 10.7 & 23.9 & 14.4 & 22.3 & 13.7 \\
\hline Esophagus & $*$ & ${ }^{*}$ & $*$ & 4.4 & 1.7 & 0.9 \\
\hline Kidney \& Renal Pelvis & $(16.7)^{* *}$ & $*$ & $*$ & 6.0 & 5.9 & 5.5 \\
\hline Larynx & $*$ & * & $*$ & 2.5 & 1.5 & 0.7 \\
\hline Leukemias & * & * & 7.2 & 6.8 & 7.9 & 6.4 \\
\hline Liver \& IBD & $*$ & $*$ & $*$ & 2.4 & 1.5 & 2.6 \\
\hline Lung \& Bronchus & 50.6 & * & 43.1 & 44.2 & 41.5 & 19.5 \\
\hline Non-Hodgkin's Lymphoma & $*$ & $*$ & ${ }^{*}$ & 7.6 & 12.0 & 9.1 \\
\hline Hodgkin's Disease & * & * & $*$ & 2.0 & 2.6 & 1.6 \\
\hline Melanoma & $*$ & $*$ & $*$ & 0.7 & 10.1 & 3.2 \\
\hline Multiple Myeloma & $*$ & * & * & 7.4 & 3.2 & 3.0 \\
\hline Nasopharynx & * & $*$ & $*$ & $*$ & 0.2 & $*$ \\
\hline Oral Cavity & * & $*$ & $*$ & 5.8 & 5.8 & 2.7 \\
\hline Ovary & $*$ & 17.5 & 11.8 & 10.2 & 15.8 & 11.4 \\
\hline Pancreas & $*$ & $*$ & 8.7 & 11.5 & 7.4 & 6.9 \\
\hline Stomach & ${ }^{*}$ & $*$ & 13.0 & 7.6 & 4.4 & 8.0 \\
\hline Thyroid & $*$ & $*$ & 9.1 & 3.3 & 6.5 & 6.2 \\
\hline Urinary Bladder & $*$ & $*$ & $*$ & 5.8 & 7.8 & 4.3 \\
\hline Gallbladder & $*$ & 13.2 & $*$ & $*$ & $*$ & $*$ \\
\hline
\end{tabular}

Note: Rates are "average annual" per 100,000 population, age-adjusted to 1970 U.S. standard.

* = Rate not calculated when fewer than 25 cases; data source for American Indians is New Mexico.

( ) $)^{* *}$ Generally rates based on fewer than 25 cases are inchuded only for the top five most frequently diagnosed cancers for etbnic group. These rates may be subject to greater variability than other rates which are based on larger numbers.

ONS $=$ other nervous system; IBD $=$ intrabepatic bile duct .

Rates for gallbladder appear only for American Indian women.

Source: SEER Program, National Cancer Institute (see Glossary for additional information). Miller et al., 1996 (15).

rates among the various Native American subpopulation groups (i.e., American Indians, Alaska Natives, and Native Hawaiians). As shown in Table 1, for the period 1988 through 1992, American Indian men and women in the State of New Mexico have among the lowest reported cancer incidence rates for all sites of any ethnic group. Also, American Indian mortality rates in New Mexico are among the lowest 
TABLE 2: SEER Mortality Rates, Men, 1988-1992

\begin{tabular}{|c|c|c|c|c|c|c|}
\hline Cancer Site & $\begin{array}{l}\text { Alaska } \\
\text { Native }\end{array}$ & $\begin{array}{c}\text { American } \\
\text { Indian }\end{array}$ & Hawaiian & Black & White & Hispanic \\
\hline All Sites & 225.0 & 123.0 & 239.0 & 319.0 & 213.0 & 129.0 \\
\hline Brain \& ONS & $*$ & $*$ & $*$ & 3.1 & 5.4 & 3.0 \\
\hline Colon \& Rectum & 27.2 & $(8.5)^{* *}$ & 23.7 & 28.2 & 22.9 & 12.8 \\
\hline Esophagus & $*$ & $*$ & $*$ & 14.8 & 5.3 & 3.4 \\
\hline Kidney \& Renal Pelvis & $(13.4)^{* *}$ & * & * & 5.1 & 5.0 & 3.7 \\
\hline Larynx & $*$ & * & $*$ & 5.6 & 2.3 & 1.9 \\
\hline Leukemias & $*$ & $*$ & 7.8 & 8.0 & 8.5 & 5.1 \\
\hline Liver & * & $(11.2)^{* *}$ & 9.2 & 6.6 & 3.8 & 5.9 \\
\hline Lung \& Bronchus & 69.4 & $(10.4)^{* *}$ & 88.9 & 105.6 & 72.6 & 32.4 \\
\hline Non-Hodgkin's Lymphoma & $*$ & $*$ & 8.8 & 5.8 & 8.1 & 5.3 \\
\hline Hodgkin's Disease & * & * & $*$ & 0.7 & 0.7 & 0.6 \\
\hline Melanoma & * & * & $*$ & 0.5 & 3.4 & 0.8 \\
\hline Multiple Myeloma & $*$ & $*$ & $*$ & 7.3 & 3.4 & 2.7 \\
\hline Nasopharynx & $(11.6)^{* *}$ & * & $*$ & 0.6 & 0.3 & 0.3 \\
\hline Oral Cavity & $*$ & $*$ & $*$ & 8.7 & 3.8 & 2.7 \\
\hline Pancreas & * & $*$ & 12.8 & 14.4 & 9.7 & 7.1 \\
\hline Prostate & $*$ & 16.2 & 19.9 & 53.7 & 24.1 & 15.3 \\
\hline Stomach & $(18.9)^{* * *}$ & $(11.2)^{* *}$ & 14.4 & 13.6 & 6.1 & 8.4 \\
\hline Testis & * & * & $*$ & 0.1 & 0.3 & 0.2 \\
\hline Thyroid & $*$ & * & $*$ & 0.3 & 0.3 & 0.2 \\
\hline Urinary Bladder & * & * & * & 4.8 & 5.8 & 2.8 \\
\hline
\end{tabular}

Note: Rates are "average annual" per 100,000 population, age-adjusted to 1970 U.S. standard.

* = Rate not calculated when fewer than 25 deatbs; data source for American Indians is New Mexico.

$(j * *=$ Rates based on fewer than 25 deaths are included only for the top five most common types of cancer death for ethnic group. These rates may be subject to greater variability than other rates which are based on larger numbers.

ONS = other nerious system; $I B D=$ intrabepatic bile duct.

Source: SEER Program, National Cancer Institute (see Glossary for additional information), Miller et al., 1996 (15).

reported (Table 2). Because the cancer rates reported in Tables 1 and 2 are for American Indians in New Mexico only, these are not necessarily representative of those for American Indians living in other regions of the country.

The leading causes of cancer mortality in American Indian men for the period 1988 through 1992 were prostate cancer (16.2 per 100,000 population), followed by cancers of the stomach and liver (both 11.2), lung and bronchus (10.4) (hereinafter referred to as lung), and colon and rectum (8.5) (Table 2) (15). The leading causes of cancer mortality in American Indian women were gallbladder cancer (8.9 per 100,000 population), followed by cancers of the breast (8.7), cervix (8.0), pancreas (7.4), and ovary (7.3) (15).

Table 1 also shows that there was higher incidence of kidney cancer among American Indian 
TABLE2: SERP Mortin Rates, Women, 998.1992 (continued)

\begin{tabular}{|c|c|c|c|c|c|c|}
\hline Cancer Site & $\begin{array}{l}\text { Alaska } \\
\text { Native }\end{array}$ & $\begin{array}{c}\text { American } \\
\text { Indian }\end{array}$ & Hawailan & Black & White & Hispanic \\
\hline All Sites & 179.0 & 99.0 & 168.0 & 168.0 & 140.0 & 85.0 \\
\hline Brain \& ONS & $*$ & $*$ & * & 2.1 & 3.7 & 2.0 \\
\hline Breast & $(16.0)^{* *}$ & $(8.7)^{* *}$ & 25.0 & 31.4 & 27.0 & 15.0 \\
\hline Cervix & $*$ & $(8.0)^{* *}$ & $*$ & 6.7 & 2.5 & 3.4 \\
\hline Colon \& Rectum & 24.0 & $*$ & 11.4 & 20.4 & 15.3 & 8.3 \\
\hline Corpus Uteri & $*$ & * & 8.4 & 6.0 & 3.2 & 2.3 \\
\hline Esophagus & $*$ & $*$ & $*$ & 3.7 & 1.2 & 0.7 \\
\hline Kidney \& Renal Pelvis & $(7.4)^{* *}$ & * & * & 2.2 & 2.3 & 1.7 \\
\hline Larynx & $*$ & $*$ & * & 0.9 & 0.5 & 0.2 \\
\hline Leukemias & $*$ & $*$ & $*$ & 4.6 & 5.0 & 3.4 \\
\hline Liver \& IBD & $*$ & * & $*$ & 2.7 & 1.8 & 2.8 \\
\hline Lung \& Bronchus & 45.3 & $*$ & 44.1 & 31.5 & 31.9 & 10.8 \\
\hline Non-Hodgkin's Lymphoma & $*$ & * & $*$ & 3.4 & 5.3 & 3.6 \\
\hline Hodgkin's Disease & * & * & * & 0.4 & 0.4 & 0.3 \\
\hline Melanoma & $*$ & $*$ & * & 0.4 & 1.7 & 0.5 \\
\hline Multiple Myeloma & * & * & * & 5.0 & 2.2 & 1.8 \\
\hline Nasopharynx & $*$ & $*$ & $*$ & 0.2 & 0.1 & 0.1 \\
\hline Oral Cavity & * & $*$ & $*$ & 2.1 & 1.5 & 0.7 \\
\hline Ovary & $*$ & $(7.3)^{* *}$ & 7.3 & 6.6 & 8.1 & 4.8 \\
\hline Pancreas & $(15.5)^{* *}$ & $(7.4)^{* *}$ & 9.1 & 10.4 & 6.9 & 5.2 \\
\hline Stomach & * & $*$ & 12.8 & 5.6 & 2.8 & 4.2 \\
\hline Thyroid & $*$ & $*$ & $*$ & 0.4 & 0.3 & 0.5 \\
\hline Urinary Bladder & $*$ & $*$ & $*$ & 2.4 & 1.7 & 0.9 \\
\hline Gallbladder & $*$ & $(8.9)^{* *}$ & $*$ & $*$ & $*$ & $*$ \\
\hline
\end{tabular}

Note: Rates are "average annual" per 100,000 population, age-adjusted to 1970 U.S. standard. * = Rate not calculated when fewer than 25 deaths; data source for American Indians is New Mexico.

( ) * Rates based on fewer than 25 deaths are included only for the top five most common types of cancer death for ethnic group. These rates may be subject to greater variability than other rates which are based on larger numbers.

ONS = other nervous system; IBD = intrahepatic bile duct.

Rates for gallbladder appear only for American Indian women.

SOurce: SEER Program, National Cancer Institute (see Glossary for additional information). Miller et al., 1996 (15).

men compared with U.S. white males. For American Indian women, incidence rates for cancers of the cervix, ovary, and gallbladder were higher than those for the U.S. white female population (15).
A greater cancer burden is reported here for Alaska Natives and Native Hawaiians. Also, because cancer rates vary by geographic region and among tribes, understanding the cancer control and prevention needs of Native Americans is complicated (16). For 
example, the leading causes of cancer mortality in Alaska Native men were lung cancer (69.4), followed by cancers of the colon and rectum (27.2), stomach (18.9), kidney (13.4), and nasopharynx (11.6) (15). The leading cause of cancer mortality in Alaska Native women was lung cancer (45.3); next are cancers of the colon and rectum (24.0), breast (16.0), pancreas (15.5), and kidney (7.4) (Table 2).

In comparison with the U.S. white population, Alaska Natives have excessive incidence of cancers of the colon and rectum, lung, and stomach for men, and all cancers combined and cancers of the cervix, colon and rectum, and lung for women (Table 1). Moreover, Alaska Natives have excessive cancer mortality rates for all cancers combined and cancers of the colon and rectum for men, and all cancers combined and cancers of the colon and rectum and lung for women, compared with the U.S. white population (15) (Table 2).

There was a dramatic increase in lung cancer during the period 1989 through 1993 (nearly fourfold in women), which is primarily attributable to the high rate of cigarette smoking among Alaska Natives (17). lung cancer mortality began to increase in the 1970 s in Alaska Native men, while the 1980s marked an increase among Alaska Native women. Researchers assume that the high rate of tobacco use in this population had been present for at least 20 years.

The pattern of cervical cancer in Alaska Natives differs from that of other female cancers. Cervical cancer rates rose from 1969 to 1983, then began to decline during the 5-year period from 1984 through 1988. Although rates for cervical cancer are still nearly twice that of the U.S. white population, extensive efforts have been undertaken, particularly since 1991, to increase Pap smear screening for all women and followup and treatment for women with abnormal Pap smears (17).

The leading cause of cancer mortality in Native Hawaiian men is lung cancer (88.9), followed by cancers of the colon and rectum (23.7), prostate (19.9), stomach (14.4), and pancreas (12.8) (Table 2). The leading cause of cancer mortality in Native Hawaiian women is lung cancer (44.1), which is followed by cancers of the breast (25.0), stomach (12.8), colon and rectum (11.4), and pancreas (9.1) (15)

Among the Native Hawaiian population, compared with the U.S. white population, there is an excessive incidence of cancer of the esophagus, stomach, lung, and pancreas in men (Table 1). In Native Hawaiian women, there is an excessive incidence of cancers of the cervix, corpus uteri, lung, pancreas, stomach, and thyroid. In addition, Native Hawaiian men have higher cancer mortality rates, compared with the U.S. white population, for all cancers combined as well as cancers of the colon and rectum, liver, lung, pancreas, non-Hodgkin's lymphoma, and stomach (Table 2). For Native Hawaiian women, there is an excess mortality of all cancers combined and cancers of the corpus uteri, lung, pancreas, and stomach (15).

As shown in Table 1, Native Hawaiians rank first or second in cancer incidence for cancers of the esophagus (men), lung, breast, and corpus uteri (Table 1); they also rank first or second in cancer mortality for all cancers combined and cancers of the liver (men), lung, corpus uteri, non-Hodgkin's lymphoma (men), ovary, pancreas (men), and stomach (15) (Table 2).

Data on cancer rates among Native Americans are limited. Moreover, data are limited for cancer risk factors and cancer prevention and control services. These limitations in research data led to the funding of the avoidable mortality and primary prevention studies. The following seven chapters address these limitations. After reading this monograph, the reader should have an understanding of what each of these studies accomplished and how the accomplishments were achieved. The studies reported on in this monograph provide important data on methods, approaches, and findings of the NCI-supported Native American research studies. Each study provides valuable data useful for planning interventions in Native American communities.

\section{REFERENCES}

1. Byar DP, Freedman LS. The importance and nature of cancer prevention trials. Semin Oncol 1990;17:413-24

2. Campbell DT, Stanley JC. Experimental and quasi-experimental designs for research. Chicago: Rand McNally College Publishing Company, 1966:24-5.

3. U.S. Department of Health and Human Services. Report of the Secretary's Task Force on Black and Minority Health. Vol. III: Cancer. 
Washington, DC: U.S. Government Printing Office, January 1986.

4. LeMaster PL, Connell CM. Health education intervention among Native Americans: a review and analysis. Health Educ Q 1994;21:521-38.

5. Broussard BA, Johnson A, Himes JH, Story $M$, Fichtner R, Hauck F, et al. Prevalence of obesity in American Indians and Alaska Natives. Am J Clin Nutr 1991;53(6 Suppl):1535S-42S.

6. Olsen SJ, Frank-Stromborg M. Cancer prevention and early detection in ethnically diverse populations. Semin Oncol Nurs 1993;9: 198-209.

7. U.S. Department of Health and Human Services. Tobacco use among U.S. racial/ethnic minority groups-African Americans, American Indians and Alaska Natives, Asian Americans and Pacific Islanders, and Hispanics: a report of the Surgeon General. Atlanta, GA: U.S. Department of Health and Human Services, Centers for Disease Control and Prevention, National Center for Chronic Disease Prevention and Health Promotion, Office on Smoking and Health, 1998:90.

8. U.S. Department of Health and Human Services. Tobacco use among U.S. racial/ethnic minority groups--African Americans, American Indians and Alaska Natives, Asian Americans and Pacific Islanders, and Hispanics: a report of the Surgeon General. Atlanta, GA: U.S. Department of Health and Human Services, Centers for Disease Control and Prevention, National Center for Chronic Disease Prevention and Health Promotion, Office on Smoking and Health, 1998:88.

9. Oregon Research Institute, Northwest Portland Area Indian Health. Tribal tobacco policy workbook. Portland, OR: Oregon State University, February 1996.

10. Baquet $C$, Ringen $K$. Cancer among blacks and other minorities: statistical profiles. Publication No. 86-2785. Bethesda, MD: National Cancer Institute, March 1986.
11. Schinke SP, Schilling RF, Gilchrist LD, Ashby MR, Kitajima E. Pacific Northwest Native American youth and smokeless tobacco use. Int $J$ Addict 1987:22:881-4.

12. The Network for Cancer Control Research Among American Indian and Alaska Native Populations. Cancer prevention and control research among American Indians and Alaska Natives: a strategic plan for State public health agencies. Bethesda, MD: National Cancer Institute, November 1994.

13. U.S. Department of Health and Human Services. Proceedings of the National Conference of High Blond Pressure Control in Native American Communities. NIH Publication No. 79-1960. Bethesda, MD: National Institutes of Hedth, April 1977.

14. Rhoddes ER, Hammond J, Welty TK, Handler $\mathrm{AO}$, Amler RW. The Indian burden of illness and future health interventions. Public Health Rep 1987;102:361-8.

15. Miller BA, Kolonel LN, Bernstein L, Young J Jr, Swanson G, West D, et al., editors. Racial/ethnic patterns of cancer in the United States 19881992. SEER monograph. NIH Publication No. 984104. Bethesda, MD: L.S. Department of Health and Human Services, National Institutes of Health, National Cancer Institute, Cancer Control Research Program, 1998.

16. Indian Health Service, Division of Program Statistics. Regional differences in Indian health, 1990. Rockville, MD: U.S. Department of Health and Human Services, Public Health Service, Indian Health Service, Office of Planning, Evaluation, and Legislation, Division of Program Statistics, 1990.

17. Lanier AP, Kelly JJ, Smith B, Harpster AP, Harvey $\mathrm{T}$, Amadon $\mathrm{C}$, et al. Alaska Native cancer update: incidence rates 1989-1993. Cancer Epidemiol Biomarkers Prev 1996;5:749-51. 


\section{AUTHORS}

\section{Claudia Sanchez Glover, M.H.S.}

Health Statistician

Office of Special Populations Research National Cancer Institute

Executive Plaza South, Suite 320

9000 Rockville Pike

Bethesda, MD 20892

Tel: (301) 496-8589

Fax: (301) 435-9225

E-mail: cg51c@nih.gov

\section{Felicia Schanche Hodge, Dr.P.H.}

Director

Center for American Indian Rescarch and Education 1918 University Avenue, Suite 2A

Berkeley, CA 94704

Tel: (510) 843-8661

Fax: (510) 843-8611

E-mail: feliciash@aol.com 


\title{
Chapter 2 \\ The Alaska Native Women's Health Project To Reduce Cervical Cancer
}

\author{
Anne P. Lanier, Janet Kelly, and James Berner
}

\begin{abstract}
ervical cancer rates in Alaska Natives have consistently exceeded those of white women in the United States. To reduce morbidity and mortality from cervical cancer, the Women's Health Project (WHP) was created for Alaska Native women in Anchorage to identify knowledge and attitudes regarding cancer and cancer screening; determine frequency of Pap tests among Native women; and increase cervical cancer screening rates. After interviewing each participant, a special evening comprehensive women's preventive health clinic was established with all women providers. All women participants received counseling about cancer screening, reminders of their appointments, and assistance with scheduling. Results of tests conducted at each visit were provided to each participant. After the WHP interventions, improvements occurred in knowledge about cancer and in cervical cancer screening rates. The project developed a culturally relevant brochure and videotape to continue to educate and encourage women to participate in cancer screening after the grant period ended. These materials were made available statewide to Native and non-Native Alaskans.

A review of medical records showed that fewer than 16 percent of women over age 20 reported having a Pap test annually in the 3 years before the project began. Among women who expressed a gender preference for a provider, nearly all preferred a female for women's preventive health services. A high percentage wanted to receive the result of the Pap test even if findings were normal. Most also wanted a written or phone reminder of their annual appointment for women's preventive health services. The National Cancer Institute $(\mathrm{NCl})$ cooperative agreement helped identify opportunities for enhancements in cancer screening services for Alaska Native women, many of which have been incorporated in services offered today.
\end{abstract}

\section{BACKGROUND}

In the 1980s, Alaska Native women (Eskimo, Indian, and Aleut) had the highest rates of invasive cervical cancer incidence and mortality among all minority groups in the United States $(1-3)$. Incidence rates were two to four times greater than those of whites in the United States (Figure 1). Alaska Natives, by treaty with the U.S. Government, are entitled to receive comprehensive healthcare. Since 1954, provision of healthcare has been the responsibility of 
FIGURE I: Age-Adjusted Incidence Rates for Invasive Cervical Cancer for Alaska Natives

Compared to U.S. Whites and Biacks

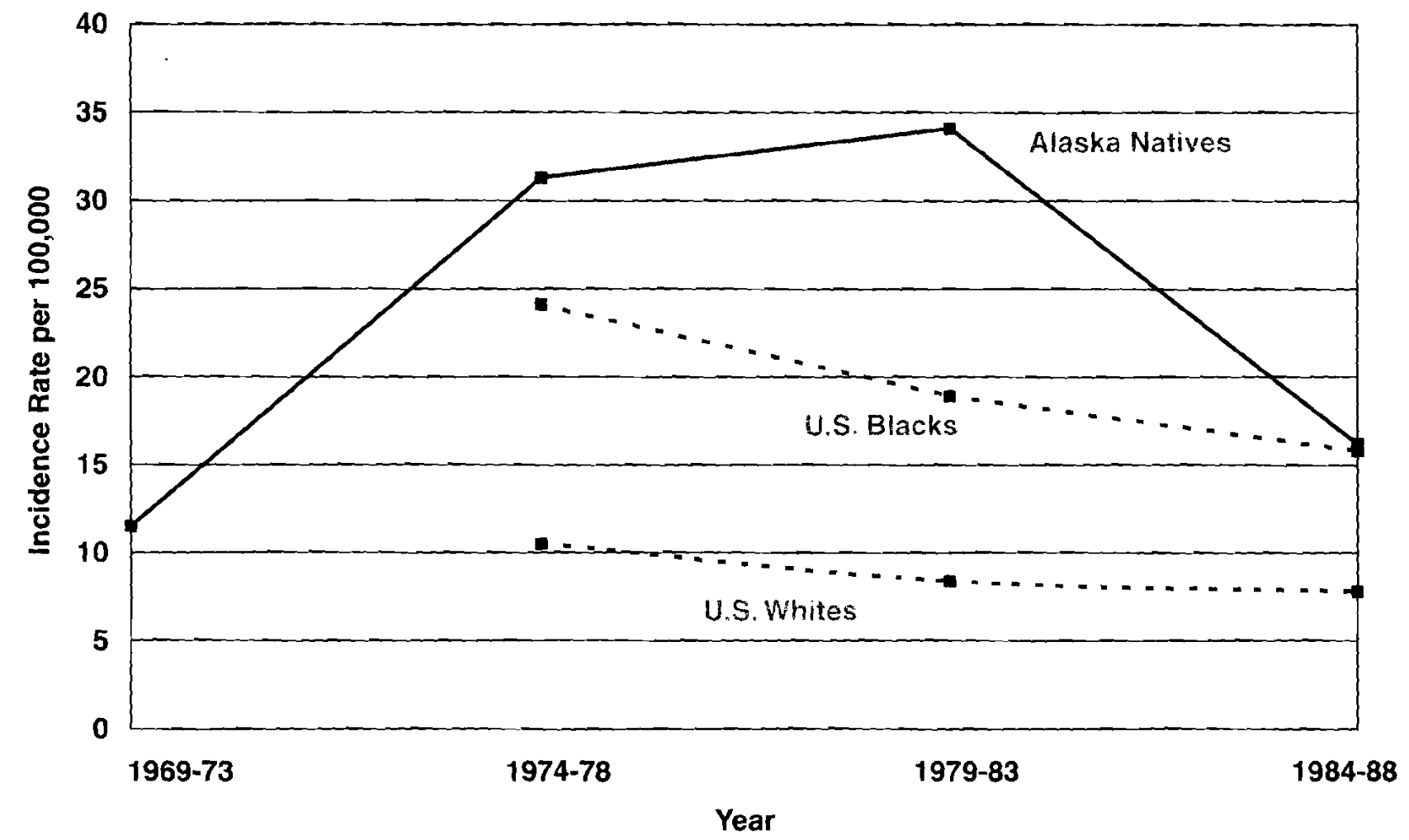

" U.S. data based on NCI's Surveillance, Epidemiology, and End Results Program.

the Indian Health Service. Tribal and regional Native health consortia now manage nearly all Native healthcare facilities and services in Alaska.

Because of high cervical cancer rates, annual Pap screening has been consistently recommended for Native women in Alaska. Until recently, no accurate information was available on how frequently women received a Pap test. However, from the total number of Pap tests done by the contract laboratory, it was estimated that only 45 to 65 percent of eligible women were actually screened in a given year. No information existed on how knowledgeable Alaska Native women were about cervical cancer, its causes, and ways to prevent it. In addition, there was no documentation of what Alaska Native women knew about early detection of cervical cancer (the Pap test), their knowledge of treatment of cancer of the cervix and related cervical disease, and their attitudes about cancer screening services that were provided. Therefore, with support from an NCI cooperative agreement in 1989, the WHP was undertaken. The overall goals of the WHP were to reduce morbidity and mortality from cervical cancer among Alaska Native women. The project objectives were to (1) assess knowledge and attitudes about cervical cancer, its causes, early detection, and treatment; (2) determine the frequency (prevalence) of cervical cancer screening; (3) assess attitudes and satisfaction regarding cancer prevention services, particularly the Pap test; (4) identify barriers to preventive healthcare; (5) increase availability of women's health services, particularly services offered by women practitioners; (6) improve followup of women with abnormal Pap tests; and (7) develop 
culturally appropriate educational materials. The project focused primarily on cervical cancer. However, because WHP recognized the importance of addressing this in the context of comprehensive women's health services, the project also addressed breast cancer, sexually transmitted diseases, and smoking.

\section{METHODS}

The original project design included three phases to last a total of 5 years. The 1-year startup phase included development of the interview instrument and medical chart review abstract form. Phase 2, intervention, was to include 3 years of clinic services, outreach, and education. The final year focused on data analysis and reporting. The project was reduced to just over 4 years, markedly reducing the intervention. All participants had the intervention clinic and activities available to them for 15 months.

Women were eligible to participate in WHP if they met the following criteria: were age 20 or older at the start of the project, resided in the Anchorage area within a reasonable distance from the Alaska Native Medical Center (ANMC), were Alaska Native and eligible to receive healthcare from the Indian Health Service, and had at least one clinic visit to ANMC in the 3 years prior to the project. Random samples of women were chosen from each of the 10 age groups from 20 to 65 years and older. The total adult Alaska Native population in Anchorage in the 1990 census was 14,569. Representatives of all Alaska Native ethnic groups reside in Anchorage.

The ANMC maintains a computerized database that includes registration and medical encounter information on all persons who receive care. From this database, the research team identified 5,889 women who met the criteria. The project goal was to enroll 500 women randomly selected from the eligible population and include equal numbers of older and younger women.

Alaska Native women who agreed to participate were intervicwed by means of a knowledge, attitude, and behavior survey (KAB1). Then WHP assisted these women in scheduling an appointment with the women's health or family practice clinic in the daytime or with the project's women's health clinic held in the evening. A total of 481 women consented to be interviewed. The WITP clinic was conducted only by women providers who offered hour-long appointments and comprehensive screening and education services. If the participant was not due for a Pap test at the time of the interview, she received a reminder by mail and then by phone when she was due. Thus, the intervention included provision of services at the WHP evening clinic for patients who chose that clinic instead of routine daytime clinic services, whereas all participants received extensive education about cancer screening, assistance in making appointments, and reminders about appointments.

At the end of the project, 200 women participated in a second face-to-face interview about knowledge, attitudes, and behaviors (KAB2). All women who participated received $\$ 20$ to offset costs incurred at each of the two interviews. The original project design included plans to interview all participants; however, the decreased funding and project duration made it possible to administer the second interview to 200 women only. These were chosen at random from the original 481 enrollees. At the end of the study, WHP conducted a consumer satisfaction survey by phone among a random sample of 100 women who had been seen in the special WHP comprehensive health clinic held in the evening.

At the onset of the project, WHP reviewed the medical records of each of the 481 participants and collected information on demographics, reproductive history, and any Pap test noted in the chart prior to the onset of the project. Information on screening included the date(s) of the screening Pap test, results, followup date, and treatment, if any. At the end of the project, charts of each participant were reviewed again and information abstracted on any Pap test, results, and followup conducted during the project intervention period. At the beginning of WHP, an extensive review identified no appropriate educational materials relevant to cervical cancer for the Alaska Native population. Consequently, a brochure ("Pap Test") and later a video (The Birthday Gift) were developed for use with Alaska Natives. WHP designed calendars that included photographs of original Alaska Native art and stickers to remind women of preventive health appointments. The video was developed using extensive input from Native women and medical proviclers. Evaluations included administration of a pretest and 


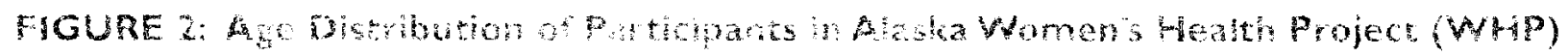

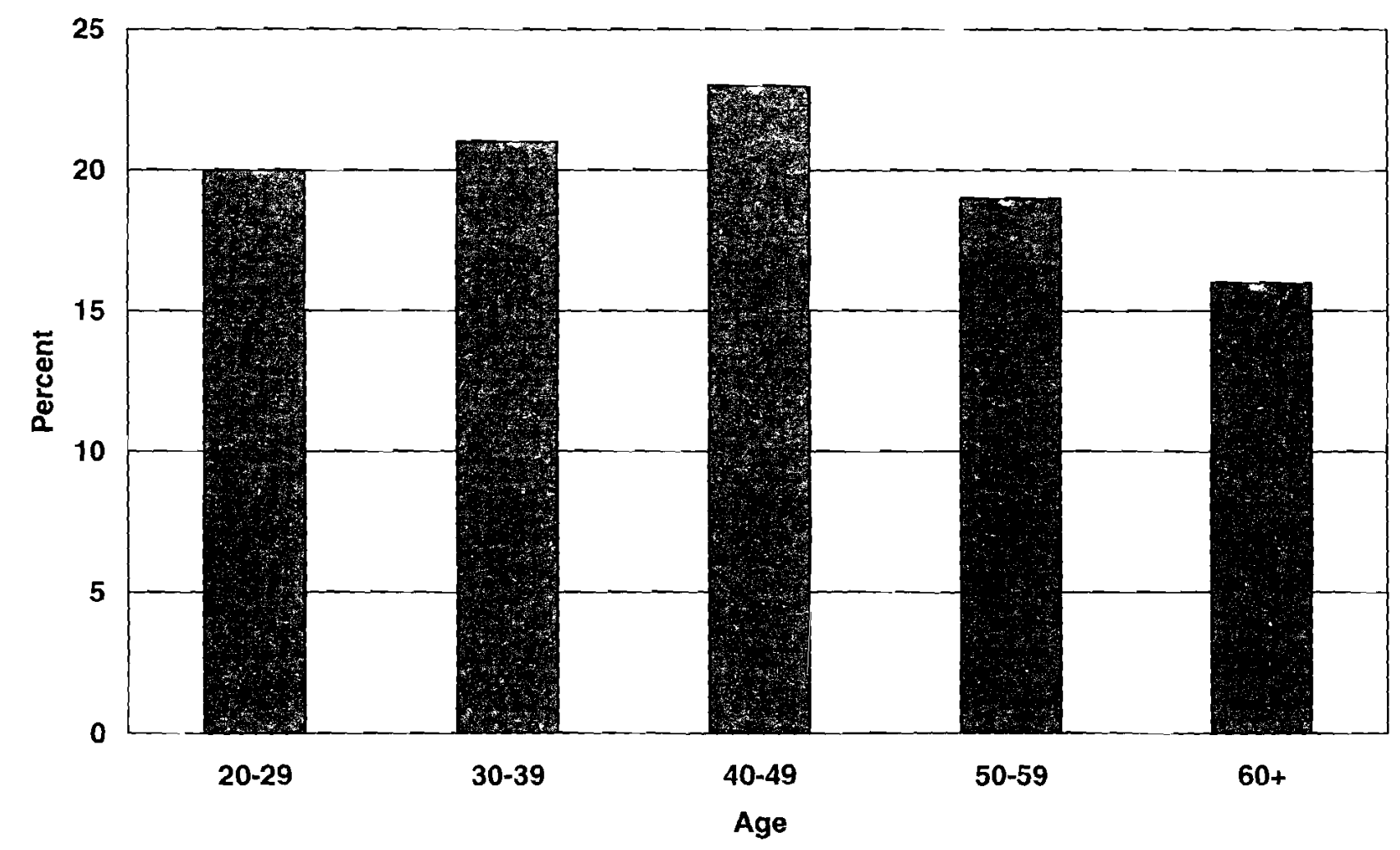

posttest to a variety of women's groups who volunteered to review the video. Results of this evaluation have been reported elsewhere (4).

The research team evaluated changes in knowledge and attitude through comparison of the number of women who responded correctly to questions asked on both the first and second interviews. Changes in behavior (Pap screening rates) were assessed through comparison of the number of Pap tests of participants before and after entry into the study, as well as by comparing the number of Pap tests of the study population with those of a control group that did not participate in the study and received no particular cervical cancer education nor encouragement and help in scheduling appointments. Information on the control group was collected as part of a student thesis for a master's degree in nursing at the University of Alaska in Anchorage. The research team also examined improvement in screening rates for those who par- ticipated in the WIIP evening clinic compared to those who received screening at routine daytime clinic appointments.

\section{RESUTS}

\section{Demography and Risk Factors}

The project design called for enrollment of 500 Anchorage Native women, 50 in each of ten 5-year age groups from age 20 to 65 and older. A total of 481 participants were enrolled. Of 611 women who were contacted, given information about the project, and invited to enroll, 79 percent participated. Only 130 of the women contacted directly declined; they are categorized as nonparticipants. The research team reviewed medical records for all participants $(n=481)$, nonparticipants $(n=130)$, and controls ( $n=250$ ). As shown in Figure 2, by design, the study included a larger proportion of older women than are in the population of Anchorage Native women, 
FIGURE 3: Distribution of Alaska Native Participants in the WHP by Ethnic Group (Aleut, Indian, and Eskimo)

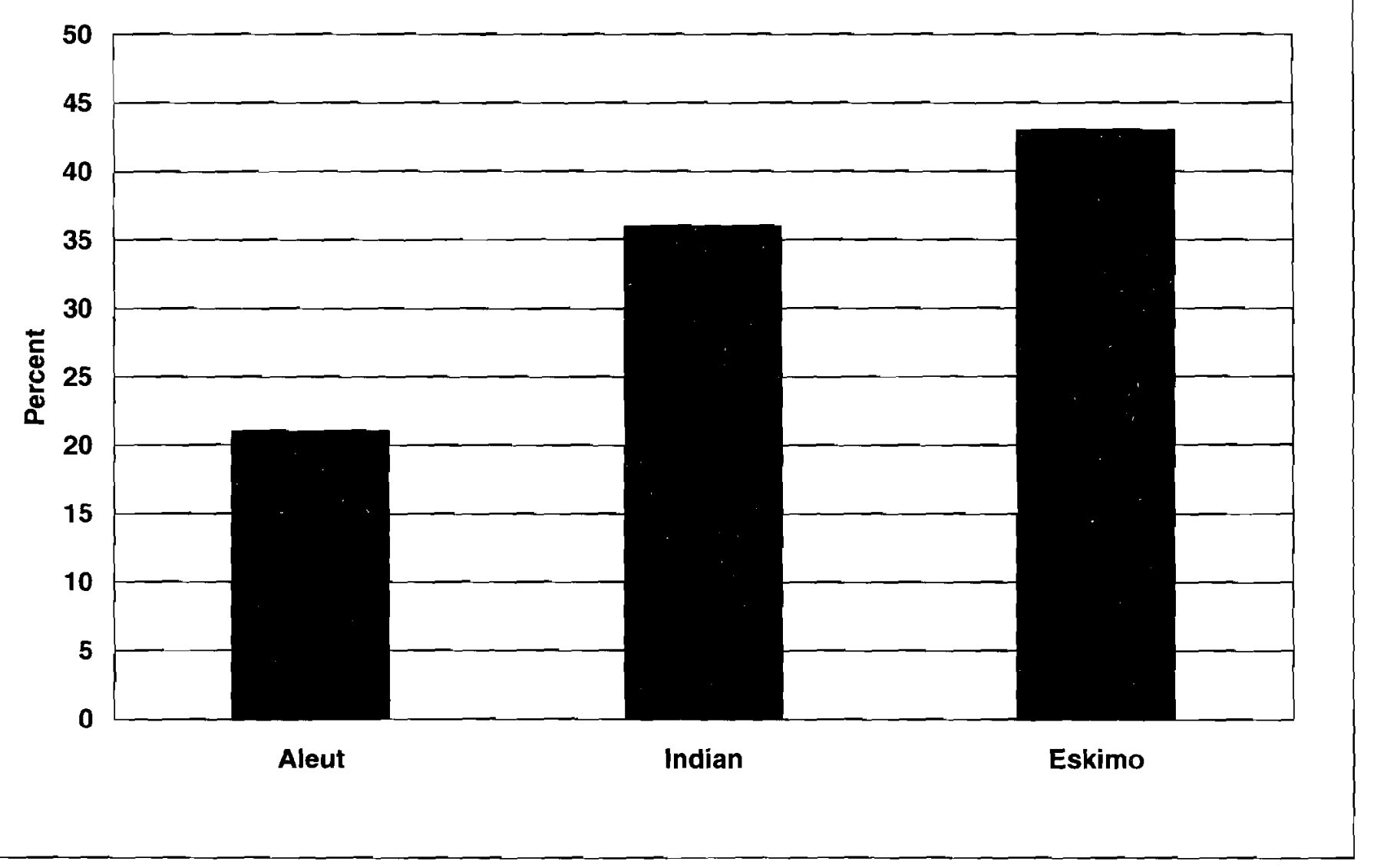

thus achieving the project goal of having nearly equal numbers of women in cach age group.

The distribution of the project sample by ethnic group (Eskimo, Indian, and Aleut) is shown in Figure 3. It approximates the ethnic distribution of the entire Anchorage Native women's population enumerated in the 1990 census. The distribution of participants by ethnicity, level of education, marital status, and number of pregnancies is shown in Table 1. Seventy-four percent of 481 women interviewed completed high school or technical training. Education was strongly correlated with age: 87 percent of 20-ycar-olds had completed high school, whereas only 63 percent of 50 -year-olds and 38 percent of 60-year-olds had completed high school (Figure 4). Nine percent had never married; 33 percent were divorced, separated, or widowed; and 58 percent were married or in a marriage-like relation- ship (Table 1). Seven percent had had no pregnancies, and 33 percent had had five or more pregnancies (Table 1). At the birth of their first full-term infant, 83 percent of the women were age 18 to 29 , 13 percent were younger than age 18 , and 4 percent were over age 29.

During the interview, women reported a variety of behaviors that potentially increase their risk for cervical cancer: 43 percent of the group were current smokers; 6 percent had two or more sex partners in the previous 6 months; 18 percent had two or more different sexually transmitted diseases (STDs) at some time in their lives; and 41 percent scored low on knowledge of methods of preventing STDs.

\section{Pap Test Prevalence}

The review of subjects' charts showed that, prior to the beginning of the study, specifically during the 3- 
TABLE 1: Distribution of Participants in the WHP by Ethnicity, Education, Marital Status, and Number of Pregnancies $(n=48 !)$

\begin{tabular}{lclc} 
Ethnicity & Percentage & Marital Status & Percentage \\
\hline Aleut & $21 \%$ & Married & $58 \%$ \\
Indian & 36 & Never Married & 9 \\
Eskimo & 43 & Divorced/separated/widowed & 33 \\
& & & \\
Education & Percentage & Pregnancy & Percentage \\
$-<$ High School & $26 \%$ & -1 None & $7 \%$ \\
High School & 28 & 1 to 4 & 60 \\
$>$ High School & 46 & 5 or more & 33 \\
\end{tabular}

FIGURE 4: Percentage of Participants Who Completed High School by Age in the WHP

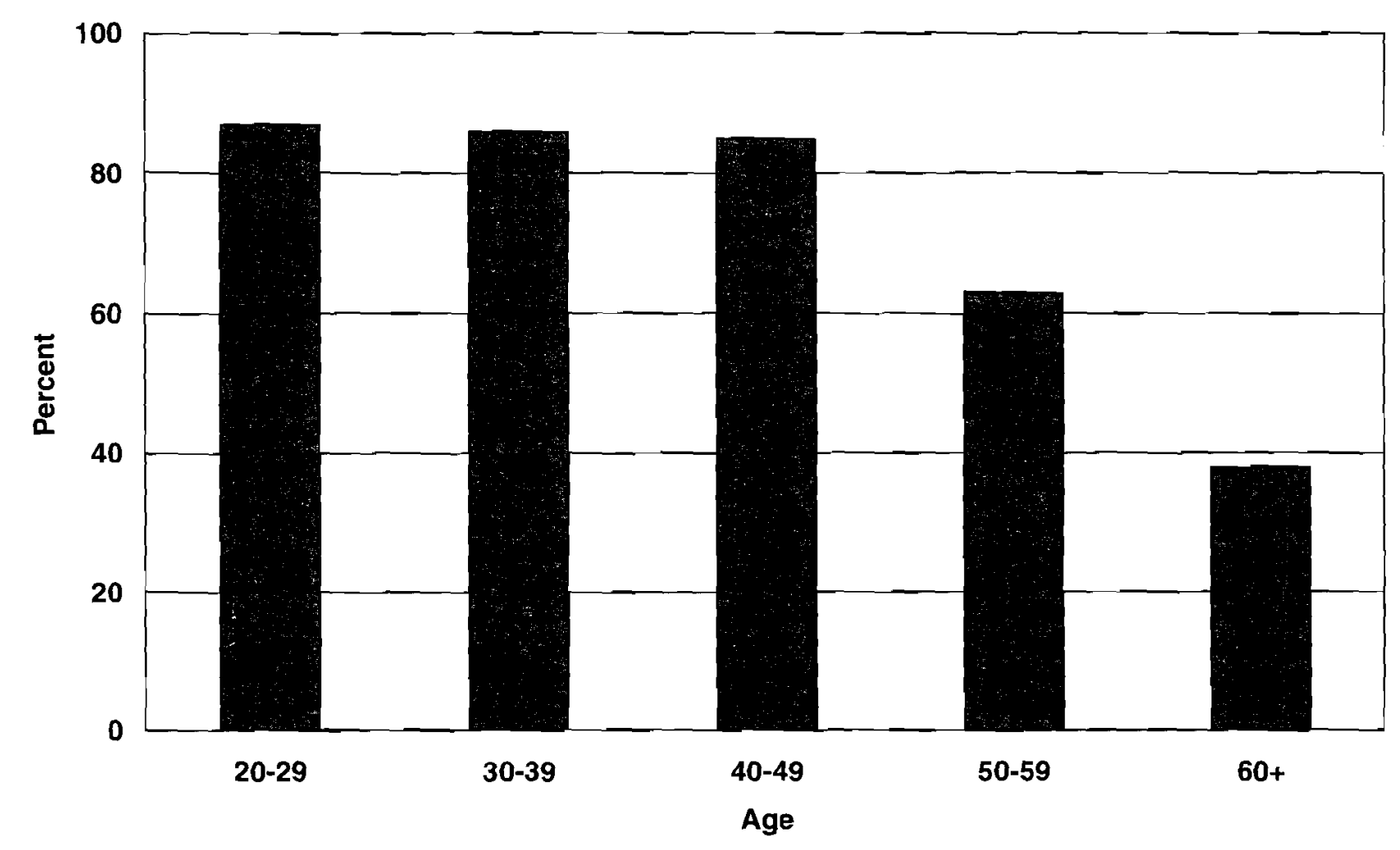


FIGURE 5: Frequency of Pap Tests Documented by Medical Record Review Among Anchorage Native Women (Participants, Nonparticipants, and Controls) in the WHPa

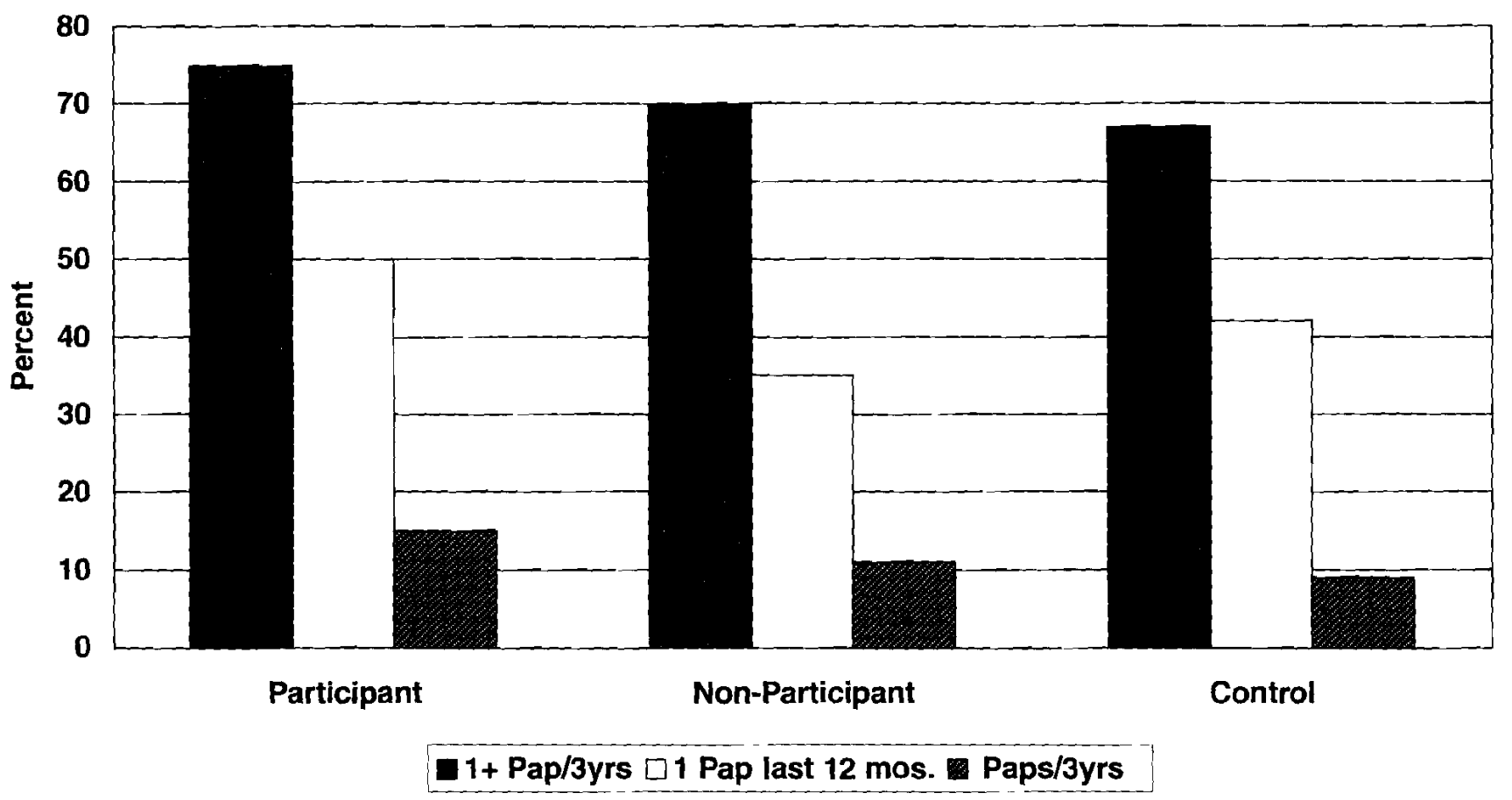

" Percentages show the number of Pap tests women received prior to cntry into the study-at least one Pap test in the 3 years prior, one Pap test in the previous year, and one Pap test in each of the 3 years prior to the initiation of the study.

year period from 1989 to 1991,75 percent of participants and 67 percent of controls had had at least one Pap test (Figure 5). However, 50 percent or fewer had a Pap test in the year just prior to initiation of the study, and fewer than 16 percent in any group had a Pap test in each of the 3 years. Therefore, more than 80 percent were not receiving Pap tests at the frequency recommended by the medical service provider

\section{Change in Knowledge}

At the initial interview (KAB1), participants were asked about cervical cancer (four questions), breast cancer (four questions), and health hazards of smoking (eight questions). Responses indicated that participants were quite knowledgeable about breast cancer screening and somewhat less knowledgeable about cervical cancer (Table 2). Although women were aware that smoking caused lung cancer and breathing disorders, they were less likely to know that smoking also caused heart disease and lowbirth-weight babies. A small percentage knew smoking could cause cervical cancer or ear problems. Despite the rather high percentage of women who were quite knowledgeable on the first interview, the percent of women who answered the questions cor- 


\begin{tabular}{|c|c|c|c|}
\hline Category & \multirow{2}{*}{$\begin{array}{l}\text { Question } \\
\text { See provider for lump }\end{array}$} & \multicolumn{2}{|c|}{ Percentage } \\
\hline & & $98 \%$ & $100 \%$ \\
\hline & All lumps not cancerous & 80 & 84 \\
\hline & Breast self-exam important & 96 & 99 \\
\hline & Annual breast exam important & 92 & 91 \\
\hline \multirow[t]{4}{*}{ Cervix } & Heard of cancer test (Pap test) & 60 & 72 \\
\hline & Correctly names test & 42 & 52 \\
\hline & Pap test if using birth control pills & 89 & 92 \\
\hline & Pap test after menopause & 85 & 92 \\
\hline \multirow[t]{6}{*}{ Hazards of Smoking } & Low-birth-weight infants & 79 & 89 \\
\hline & Breathing illness & 92 & 99 \\
\hline & Ear problems & 29 & 54 \\
\hline & Heart disease & 86 & 92 \\
\hline & Lung cancer & 99 & 99 \\
\hline & Cervical cancer & 22 & 63 \\
\hline
\end{tabular}

rectly increased between the first and second interviews (Table 2).

\section{Change in Behavior}

Table 3 shows the percentage of women who had no, one, or two or more Pap tests before and during the intervention phase of the study. The percentages are shown for both the participants and the control group (women who received no WHP education or services). Among participants, the percentage of women who had not had a Pap test declined from 48 to 20 percent. By comparison, the percentage of women in the control group who had not had a Pap test (50 percent) was the same before and after.

Women were placed in an "improved" category if they received more annual Pap tests while participating in the project than they did in the same period of time prior to their entry into the project. The authors compared women who had received services in the special WHP evening clinic with those who attended the daytime clinic. Improvement in cervical cancer screening was documented in 64 percent of women who used the evening clinic and 44 percent of those who used the daytime clinic.

Before and after project intervention, women expressed a strong preference for preventive health services to be provided by women. On a consumer satisfaction survey administered at the end of the project period, women expressed a very high level of satisfaction with the WHP clinic services.

\section{DISCUSSION}

High rates of cervical cancer among Alaskia Native women persist, despite the availability of free Pap tests and the longstanding recommendation by the Alaska Indian Health Service for annual testing. The research team conducted an intervention project in Anchorage to address women's knowledge, attitudes, and behaviors about cervical cancer screening, cancer risk factors, and women's health 
TABLE 3: Pap Test Frequency (Based on Medical Record Review) Among WHP Participants and Controls Before and After Intervention

Pap Test $=0$

Participants

Before

After

$48 \%$

$20 \%$
Pap Test $=1$

$41 \%$

$49 \%$
Pap Test $=2+$

$12 \%$

$31 \%$

Controls

Before

After
$50 \%$

$50 \%$
$42 \%$

$39 \%$
$7 \%$

$11 \%$ maintenance and preventive services.

Despite the unexpectedly short intervention phase, project objectives were met. The study documented an increase in cervical cancer screening among participants. During the intervention phase, WHP provided additional women's preventive services during extended clinic hours that would not have been available without grant support. These services and other project interventions led to a documented increase in cervical cancer screening rates among participants. WHP also documented improvements in women's knowledge of cervical cancer screening, cancer risks, and ways to reduce their own cancer risks. Much also was learned about Alaska Native women's preferences for delivery of preventive services.

Additional benefits of the project included development of an educational brochure and video, specific to Alaska Natives, about the Pap test. KAB survey results from the project also provided information for subsequent development of another brochure and several videos on breast cancer and breast cancer screening. Additional benefits included participation of more than 15 individuals, including Alaska Native women, in the design, conduct, evaluation, and reporting of the project as well as experience in developing culturally specific materials for cancer education. Some aspects of the grant supported comprehensive women's health clinic services, which were continued at ANMC and included in a cancer screening program initiated by Alaska tribal organizations funded by the Centers for
Disease Control and Prevention's National Breast and Cervical Cancer Early Detection Program.

\section{REFERENCES}

1. Lanier AP, Kelly JJ, Smith B. Amadon C, Harpster A, Peters H, et al. Cancer in the Alaska Native population: Eskimo, Aleut, and Indian. Incidence and trends 1969-1988. Alaska Med 1994;36:3-92.

2. Miller BA, Ries LAG, Hankey BF, Kosary Cl, Harras A, Devesa SS, et al. SEER Cancer Statistics Review, 1973-92. NIH Publication No. 96-2789. Bethesda, MD: National Cancer Institute, 1996.

3. Roche J, Jones M, Anderson $\mathrm{T}$, Middaugh $\mathrm{J}$. Alaska Cancer Control Plan. Anchorage: State of Alaska, Department of Health and Social Services, Division of Public Health, Section of Epidemiology, January 1994.

4. Stillwater B, Echavarria VA, Lanier AP. Pilot test of a cervical cancer prevention video developed for Alaska Native women. Public Health Rep 1995;110:211-4.

\section{ACKNOWLEDGMENTS}

The authors would like to express thanks to additional project personnel: James E. Berner, Lucy Billie, Dodie Matsko, Carol Crandall, Michelle Moran, Margaret Galovin, Barbara Stillwater, Teri Gleason, Nancy Sydnam, Pat Taylor, Caroline Kuether, Barbara Williams, Regina Kuhnley, Kendall 
Thomas, and the Aleutian Pribilof Island Association, Inc. This study, "Prevention of Cervical Cancer in Alaska Native Women," was funded by the National Cancer Institute (Grant No. U01CA52242).

\section{AUTHORS}

\section{Anne P. Lanier, M.D., M.P.H.}

Alaska Native Health Board

4201 Tudor Center Drive, Suite 105

Anchorage, AK 99508

Tel: (907) 562-6006 ext. 102

Fax: (907) 563-2001

E-mail: alanier@anhb.org
Janet Kelly, M.S., M.P.H.

P.O. Box 112275

Anchorage, AK 99511-2275

Tel: (907) 345-3035

Fax: (907) 345-3035

E-mail: kell102.wonder.em.cdc.gov

\section{James Berner, M.D.}

Alaska Area Native Health Service 4141 Ambassador Drive Anchorage, AK 99508-5928

Tel: (907) 729-3640

Fax: (907) 729-3652

E-mail: jberner@akanmc.alaska.ihs.gov 


\title{
Chapter 3 \\ Cervical Cancer Education for Native American Women in North Carolina: The North Carolina Native American Cervical Cancer Prevention Project
}

\author{
Ronny A. Bell, Mark B. Dignan, Robert Michielutte, Penny Sharp, \\ Karen Blinson, and John G. Spangler
}

\begin{abstract}
Whis chapter provides a description of the program and results from the North Carolina Native American Cervical Cancer Prevention Project (NCP), a 5-year cancer control investigation funded by the National Cancer Institute $(\mathrm{NCl})$. The study populations for NCP comprised two Native American tribes residing in different regions of North Carolina, the Eastern Band of Cherokee Indians and the Lumbee. Based on social learning theory, the intervention tested in NCP consisted of a tailored health education program. Native American lay health educators delivered the intervention to women in two personal visits in their homes. The lay health educators obtained information needed for a health risk appraisal-knowledge, behavioral intentions, cervical cancer screening history, and perceived barriers to screening-during the first visit. They aiso showed the women a videotape presentation that describes and illustrates the Pap test. During the second visit, the lay health educator provided information that addressed the situation of the women. The research team evaluated NCP using the Solomon Four-Group design. The research team collected data via personal interviews. Among Cherokee women completing the study $(n=815)$, the intervention groups had higher knowledge levels $(O R=2.18,95 \% \mathrm{Cl}=1.08,4.39)$ and were more likely to report obtaining a Pap test $(O R=2.06,95 \% \mathrm{Cl}=1.14,3.72)$ during the past year than the control groups. For the Lumbee study population $(n=854)$, significant intervention effects were found for knowledge $(O R=1.75,95 \% \mathrm{Cl}=1.04,2.95)$ and behavioral intentions $(O R=1.64$, $95 \% \mathrm{Cl}=1.08,2.49)$ but not for Pap tests. The results of this study demonstrate the effectiveness of interventions for Native American populations delivered by lay health educators.
\end{abstract}

\section{INTRODUCTION}

Overall, cancer mortality rates for Native Americans are lower than those for the U.S. population as a whole (1). However, Native American women have

much higher rates of cervical cancer mortality than other groups of women in the United States. Ageadjusted rates for cervical cancer mortality for the 12

All references to Native Americans in this chapter refer to "American Indians." 
Indian Health Service (IHS) areas from 1990 through 1992 were approximately twice that of the U.S. rate for all races (5.9 per 100,000 vs. 2.7 per 100,000). In the Nashville IHS service area, which includes North Carolina, cervical cancer mortality rates were 4.4 per 100,000 from 1990 through 1992, approximately 63 percent higher than the rate for all races in the United States (2). In North Carolina, standardized mortality ratios of cervical cancer mortality for Native American women were estimated to be 2.27 from 1968 through 1972 and 2.30 from 1978 through 1982; these rates suggest that cervical cancer mortality remains a consistent burden for this population (3).

Despite this apparent excess in cervical cancer mortality among Native American women, there is limited information available about the risk factors for cervical cancer for these women. Even less information is available about the effectiveness of culturally sensitive cervical cancer prevention programs among Native American women, particularly in North Carolina. To the authors' knowledge, prior to 1990, no education program had specifically targeted cervical cancer prevention for Native American women in North Carolina. With proper screening and avoidance of known risk factors, cervical cancer is a largely preventable disease. Therefore, effective educational interventions could provide tremendous public health benefit to women in these communities. This report clescribes the efforts of the North Carolina Native American Cervical Cancer Prevention Project (NCP) of the Wake Forest University school of Medicine (WFUSM) in developing and implementing an individualized, community-based cervical cancer education project for Native American women among the Lumbee tribe in Robeson County and the Eastern Band Cherokee in western North Carolina. The overall goal of the project was to reduce mortality from cervical cancer by increasing the proportion of women who obtained Pap tests on an annual basis. Results from the project are presented along with lessons learned by the research team.

\section{METHOOS}

\section{Description of the Target Population}

The 1990 census indicated that approximately 80,000 residents of North Carolina reported race as Native
American, representing 1.2 percent of the total State population. According to the North Carolina Commission on Indian Affairs, there are eight organized tribal groups in the State. The two largest tribes, the Eastern Band Cherokee and the Lumbee, represent about one-half of the Native American population in the State. Therefore, the research team selected two tribes as target populations for the implementation of the North Carolina project.

The Eastern Band Cherokee is a federally recognized tribe whose primary homeland is the Cherokee Indian Reservation in western North Carolina. The reservation includes tracts of land in five rural counties-Swain, Jackson, Graham, Haywood, and Cherokee-in the Appalachian Mountains in western North Carolina. The tribe includes approximately 7,000 persons, 1,400 of whom are women over age 20 . Members of the tribe receive healthcare from IHS at the Cherokee Indian Hospital in the township of Cherokee as well as from healthcare providers on contract with the tribe.

The Lumbee are a State-recognized tribe whose primary homeland is Robeson County in rural southeastern North Carolina. Approximately 40,000 Lumbees live in Robeson County, and about 10,000 are women over age 20 . The Lumbee do not have a reservation, and healthcare is provided to tribe members through traditional means: private healthcare providers; Robeson Healthcare Corporation, a nonprofit health corporation that operates on a slicing-scale schedule; and the county health department staff (the first rural public health department in the Nation is in Robeson County). Robeson County is unique in that its population is approximately one-third Native American, one-third African American, and one-third Caucasian.

\section{Community Analysis}

To gain additional insight into the people involved in the project, the research team conducted a community analysis for the areas where members of both tribes resided. The conmunity analysis involved collecting data from Federal, State, and local agencies; conducting focus groups; and holding interviews with local "gatekeepers" and additional healthcare providers serving Lumbee and Cherokee women. The results of these efforts have been published (4). 
Among both the Lumbee and Eastern Cherokee tribes, indicators suggest that the populations are generally low income. Data from the 1990 census indicate that overall annual per capita income for Native Americans in both communities is below the State average, and low education and employment remain concerns. Since the 1980s, female-headed families in Native American communities in North Carolina have rapidly increased. Most indicators were similar for both tribes, with the exception of a much larger population of women age 18 and older among the Lumbees versus the Eastern Cherokee and a somewhat higher median level of formal education (years completed) among the Eastern Cherokee compared to the Lumbee.

Reliable data describing chronic disease risk factors are not available for Native Americans in North Carolina. Little health-related research has been conducted among Native Americans in the State, and the State-based Behavioral Risk Factor Surveillance System does not include sufficient numbers of Native Americans to describe the populations adequately.

The research team conducted three focus groups among both tribes as part of a community analysis. These focus groups were directed at determining the communities' views toward health and disease, specifically cancer; their level of satisfaction with healthcare providers; and their perceptions related to cancer treatment and prevention. The focus groups revealed a common theme of the importance of family. Health was important to these women because of the role they played as providers for their immediate and extended families. The women had largely pessimistic views toward cancer: they saw cancer as a single, deadly disease that healthcare providers were generally ineffective in preventing or treating. Early detection of cancer was not scen as positive because such knowledge would only increase the time one suffered before succumbing.

The research team conducted interviews with key contacts among both populations. As part of the community analysis, interviews were conducted with business leaders, skilled and unskilled workers, teachers, and tribal and municipal officials. Results of the interviews suggested a limited knowledge of cervical cancer, skepticism toward effective treatment of cancer, and fear of harm resulting from can- cer treatment. Native American customs were not seen as a barrier to delivering cervical cancer education messages, but a strong emphasis was placed on having health educators who are acceptable to women in the community.

We also interviewed healthcare providers in both communities to gain further insight into the provision of and attitudes toward cancer prevention and treatment. The interviews revealed that providers accepted Pap tests and followup care as standard medical care. Providers also identified specific barriers to providing care, which included being in a rural area where patients had to travel extensively outside their community to receive adequate followup care.

\section{Description of the Research Team}

The direction of NCP was a joint effort of faculty in the Department of Family and Community Medicine, the Department of Public Health Sciences, and the Comprehensive Cancer Center of WFUSM. Members of the project team had previous experience with successful cervical cancer education projects in predominantly minority communities (5-8). One member of the research team centrally managed the project. Another member of the research team, a Lumbee Indian and a native of Robeson County, added credibility to the efforts of the research team by providing insights related to the target population.

The location of the two tribes in relation to project headquarters at WFUSM presented a unique challenge to the project investigators. The Eastern Band Cherokee Reservation is approximately 250 miles, and Robeson County is approximately 150 miles, from the Wake Forest Medical Center in Winston-Salem, where the project team was located during the development and implementation of the project. (Figure 1 shows the locations of the study populations in North Carolina.)

To facilitate data collection efforts, the research team hired local Native American women in both communities to serve as interviewers and educators. Educators were called "project guides" (to connote a guiding influence for project participants in learning about cervical cancer). Directly responsible to the research team, project guides received extensive training at the project headquarters at WFUSM and 


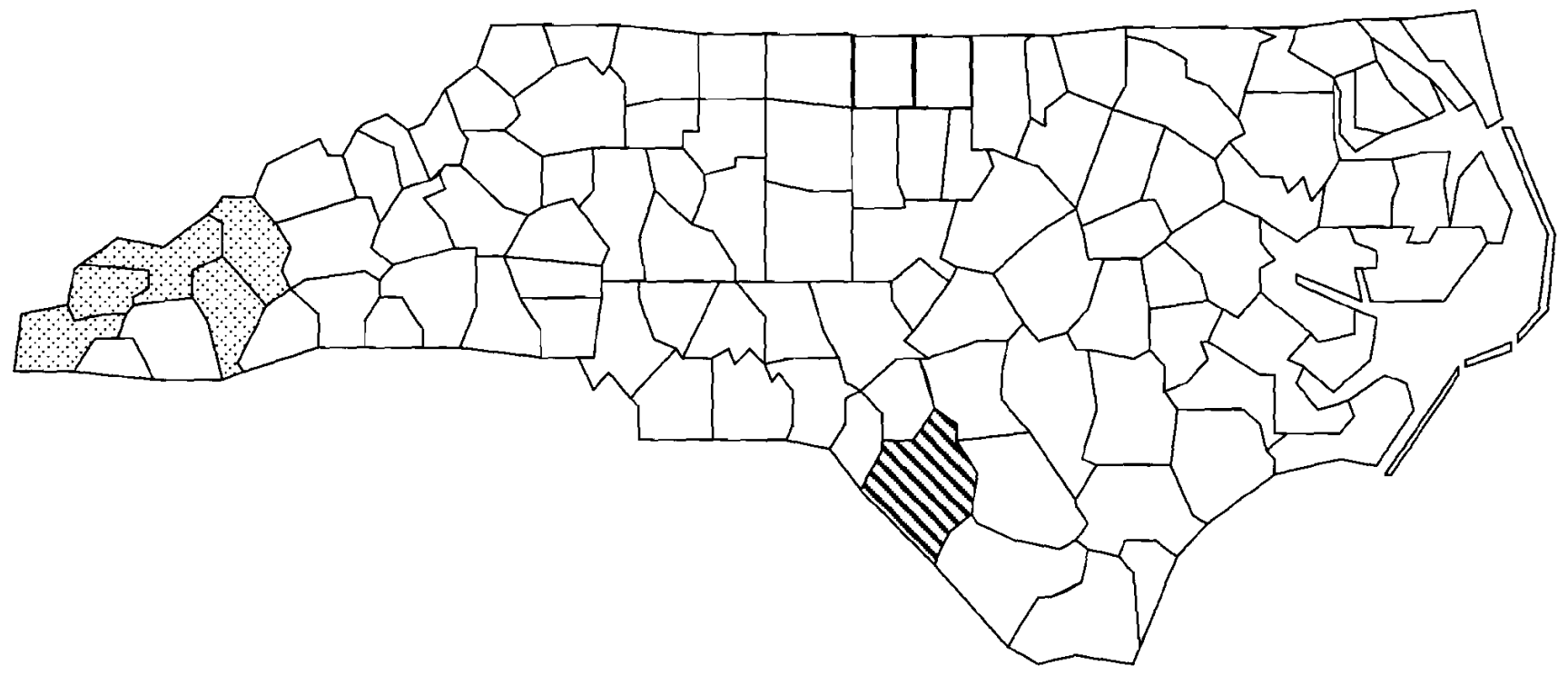

Eastern Band Cherokee Population

$\Delta$ Lumbee Population

additional training through observing participants directly in the field. The motivation for employing local Native American women for the project is described in another publication (4).

\section{Description of the Intervention}

The project instituted an individualized, communitybased approach to educate women about cervical cancer and the need for participation in cervical cancer screening. Prior to implementation of the project, the research team presented the design and purpose of NCP to the Lumbee and Cherokee tribal councils that approved them.

The development of the NCP intervention has been described in previous publications $(4,9)$. The intervention developed for NCP was an individualized health education program based on the health belief model (10), social learning theory (11), and self-efficacy (12). Two additional models included the PRECEDE (predisposing, reinforcing, and enabling causes in educational diagnosis and evaluation) model and the minority health communication model (MHCM). The health belief model indicates that perceived susceptibility to disease or injury influences action toward preventive behavjors. Social learning theory presumes that learning occurs as a process of observational modeling, which can be divided into four categories: attention, retention, motor reproduction, and motivational processes. Self-efficacy is the belief in the ability to behave in ways necessary to produce identified outcomes. Additionally, the PRECEDE model was used to identify barriers to obtaining Pap tests and followup care (13). To ensure a focus specifically on issues pertaining to Native Americans, MHCM was used (14). MHCM is divided into five phases of com- 
munication development: consulting with "experts" who are knowledgeable of the target population, consulting with community representatives, setting goals, developing communication strategies, and evaluating strategies.

The primary focus of the intervention was to provide education basic to prevention of cervical cancer. Major themes of the intervention included (1) cervical cancer can be cured if detected early; (2) the Pap test is an effective tool for early detection of cervical cancer; and (3) women need Pap tests throughout their adult lives. The intervention consisted of three components: (1) a 10-minute videotape developed by the research team, shown by the project guides in the homes of participating Native American women using a portable videocassette

\section{FIGURE 2: Copy of Printed Education Materials}

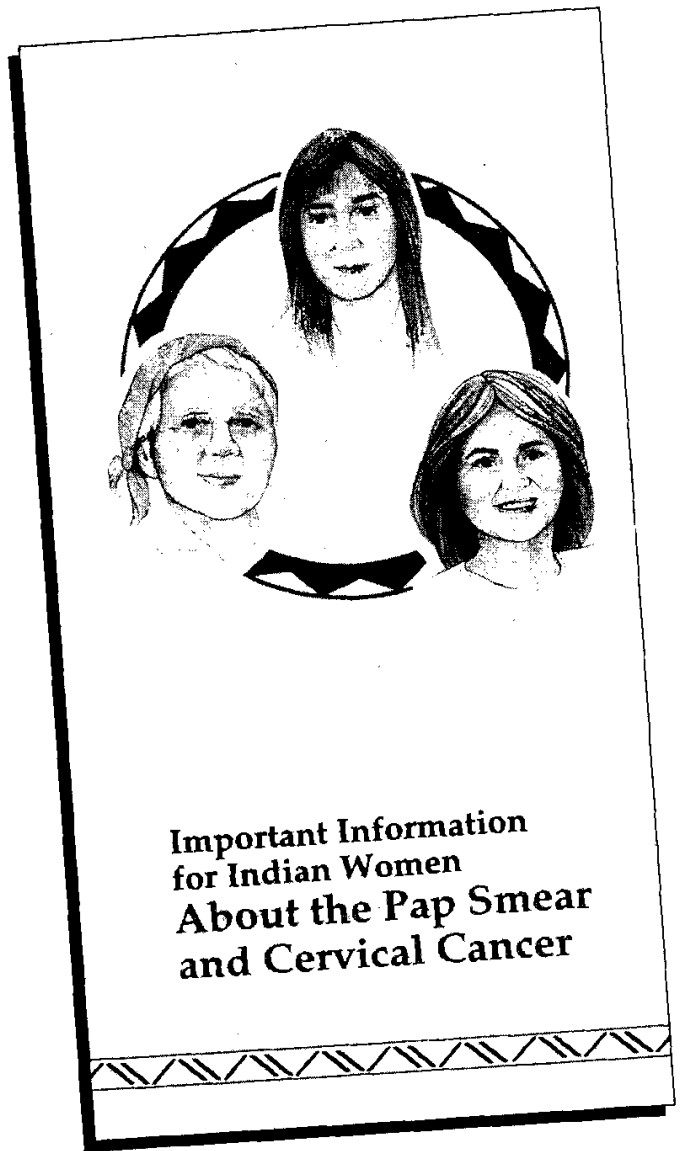

player; (2) printed educational materials developed by the research team to augment the message delivered in the video; and (3) a health risk appraisal specifically designed for Native Americans, "Finding the Way: Health Risk Appraisal for Native Americans," (15) adapted from a health risk appraisal developed by the Centers for Disease Control and Prevention. The research team pretested extensively the project video and educational materials for cultural and educational appropriateness with the project staff, focus groups, professional reviewers, physicians, nurses, and laboratory technicians. Additionally, the research team presented educational materials to tribal officials for approval. (As shown in Figure 2, the images used for the educational materials were selected to be easily identified as specific to Native American women.)

In delivering the intervention, the project guides conducted two home visits. The first visit involved administering the health risk appraisal, showing the video, and reviewing the educational materials. The second visit involved reviewing the health risk appraisal, reinforcing the message of cervical cancer prevention, and answering questions pertaining to the woman's schedule for obtaining a Pap test or followup care for abnormal results of a recent Pap test. A single project guide was employed in each study population to provide the intervention. It was decided that having one project guide deliver all of the intervention would provide greater consistency than having more than one individual involved. The research team recruited and trained women who were enrolled tribal members to be the project guides in each study population.

\section{EVALUATION}

The Solomon Four-Group Design (16) was used to test the effectiveness of the intervention (Table 1). This design allows pretesting of intervention effects. The design compares groups receiving pretesting before the intervention with groups not receiving pretesting. The comparison of results permits estimation of the extent to which the pretest influences the intervention. Women in each community were randomly assigned to one of four groups: (1) received pretest, intervention, and posttest; (2) received pretest and posttest but no intervention; (3) received intervention and posttest but no pretest; and (4) received posttest only. This method differentiated the 
potential effect of the pretest versus the intervention alone. The initial plan was to assign 250 women to each of the four groups, with a total of 1,000 women from both tribes participating in the project. These sample sizes were selected to detect a difference of 10 percentage points $(\mathrm{p}<0.05$, power $=0.80)$ between women in the intervention and control groups reporting Pap tests within the past year.

A 96-item questionnaire assessed knowledge, intentions, and behaviors pertaining to cervical cancer at the pretest and posttest phase. (The questionnaire was developed in conjunction with three other NCI-funded projects under the Avoidable Mortality From Cancer in Native American Populations Initiative.) The questionnaire was designed to be culturally sensitive in the gathering of general demographic information as well as in collecting information pertaining to health status, health beliefs, health behaviors, Native American culture, and cervical cancer risk for Native American women. Local Native American women who were recruited were trained to administer the questionnaire face to face in participants' homes. The time required for administration averaged 30 minutes.

Knowledge of cervical cancer was determined by asking if women knew that (1) the Pap test can detect cervical cancer; (2) early detection of cervical cancer increases the chance for prevention; and (3) getting followup medical care may be needed to treat cervical cancer. We assessed the degree of behavioral intention by asking women whether they felt they would get a Pap test in the next year. We assessed behavior by asking whether women had had a Pap test in the past year. Effectiveness of the intervention was determined through assessment of the differences in knowledge, intentions, and behavior among the four study groups on the posttest.

\section{DATA COLLECTION}

The uniqueness of the two tribes and the difference in their population sizes and structure required the use of different sampling strategies for each community. To recruit Eastern Cherokee women, faculty in the Department of Geography at Western Carolina University in Cullowee, NC, assisted in carrying out a complete census of all households on the Eastern Cherokee Reservation. Of the 2,223 homes listed on the census, 1,279 had at least one eligible woman in the home. From door-to-door recruitment, a total of 1,020 women (78.7 percent) from these homes agreed to participate in the project.

Lumbee women were recruited through random selection of names from the tribal enrollment database as the sampling frame. A total of 5,200 names were initially selected to obtain 1,000 women for participation in the study. The research team manually reviewed the sample to remove those names in which eligibility requirements were not met: a resident of a county other than Robeson, age under 18 years, duplicate entry, male, or deceased. A total of 1,114 eligible women were contacted by telephone; 983 ( 88 percent) agreed to participate in the project.

\section{RESULTS}

The research team analyzed the primary outcomes of knowledge, behavioral intentions, and behavior pertaining to cervical cancer for women in both tribes. Differences in knowledge between women who did and did not receive the intervention were established by a significant increase in the number of women who answered the knowledge assessment items correctly. Improved behavioral intentions were verified by a significant increase in the number of women in the intervention groups who

\section{TABLE I: Solomon Four-Group Research Design}

\begin{tabular}{cccc} 
Group & Pretest & Intervention & Posttest \\
1 & $\mathrm{O}$ & $\mathrm{X}$ & $\mathrm{O}$ \\
2 & $\mathrm{O}$ & $\mathrm{X}$ & $\mathrm{O}$ \\
3 & & & 0 \\
4 & & & 0 \\
\hline
\end{tabular}


indicated that they planned to get a Pap test in the upcoming year. Behavior change was assessed through determination of the increase in the proportion of women in the intervention groups who reported that they had a Pap test in the previous year. Exposure to the pretest also was assessed as having a potential impact on increases in knowledge, intentions, and behaviors. Results have been published for Cherokee (17) and Lumbee (18) women.

Among Cherokee women, 81.8 percent $(n=815)$ who began in the project also completed the posttest interview. The number of women enrolled and retained in each group in each study population is shown in Table 2 . Approximately 73 percent of all women who received the intervention reported having had a Pap test in the past year, compared to 64 percent of all women who did not receive the intervention (adjusted odds ratio $[\mathrm{OR}]=2.06,95 \%$ confidence interval $[\mathrm{CI}]=1.14-3.72)$. Self-reported Pap test rates among intervention participants were higher for women who received the pretest $(71.0$ percent) and who did not receive the pretest $(76.0$ percent) compared to their nonintervention counterparts 65.1 percent and 62.5 percent, respectively; chi-square $\mathrm{p}=0.008$ and 0.007 , respectively). Women who received the intervention were more likely to answer all knowledge test questions correctly at the posttest (81.3 percent), compared to those who did not receive the intervention $(76.0$ percent) (adjusted $\mathrm{OR}=2.18,95 \% \mathrm{CI} 1.08-4.39$ ) Although no differences were found among women who received the pretest, among women who did not receive the pretest, those who participated in the intervention were more likely to answer all the test items correctly (86.9 percent), compared to women who did not receive the intervention ( 76.0 percent) (chi-square $\mathrm{p}=0.012$ ). No differences were observed in intention to obtain a Pap test, according to either pretest or intervention participation.

Among Lumbee women, 87.2 percent $(n=854)$ of women completed the pretest and posttest interview. There was no significant intervention effect on the number of women who reported having reccived a Pap test in the past year. Among women who received the pretest, 74.6 percent of intervention participants reported having had a Pap test, versus 69.1 percent of control participants $(O R=1.3$,
95\% CI $=0.83-2.08$ ) (Note: Control participants were women randomized to groups 2 or 4 , as shown in Table 2). Similarly, among women who did not receive the pretest, 74.0 percent versus 66.8 percent of intervention and control participants reported having Pap tests in the past year. For knowledge, an intervention effect was observed only among women who had not received the pretest $(\mathrm{OR}=$ $1.75,95 \% \mathrm{CI}=1.04-2.95$ ). Conversely, an intervention effect for intention to obtain a Pap test was seen only among women who had received the pretest: 44.9 percent of intervention participants versus 33.2 percent of controls (OR $=1.64,95 \% \mathrm{CI}=1.08-2.49)$.

Logistic regression analysis revealed that knowledgc of the cervical cancer questions at the posttest was associated with formal education beyond the eighth gracle, age 45 to 54 years, identifying with the Native American culture, having an annual physical examination, and receiving the cervical cancer intervention. Intention to get a Pap test in the next year was associated with marital status (less likely among widowed, divorced, and separated women than among married women), formal education beyond the eighth grade, having an annual physical examination, a history of abnormal pap test results, and receiving the pretest and education program (significant interaction between pretest and education involvement). Self-reported Pap tests were associated with age (lower among women age 35 to 54 than women age 24 and younger), having an annual physical examination, and receiving the educational intervention.

\section{DISCUSSION}

Although the increases were not dramatic, NCP was generally successful in improving rates of selfreported screening for cervical cancer among Native American women. The strengths of the program appear to be linked to three primary factors: (1) the employment of local Native American women for intervention and clata collection; (2) the cultural appropriateness of the health education intervention; and (3) the nature of the intervention.

The lay health educator model involves the recruitment and training of lay individuals for roles in health education at the local level. This model has been used with varying degrees of success for several years in many projects (19). The most success- 
TABLE 2: Number of Women Enrolled and Retained by Group and Study Population

\section{Lumbee}

\begin{tabular}{ccccc} 
Group & Enrolled & Pretest & Intervention & Posttest \\
\hline 1 & 255 & 252 & 211 & 211 \\
2 & 226 & 226 & & 216 \\
3 & 266 & & 220 & 220 \\
4 & 232 & & & 207 \\
Total & 979 & 478 & 431 & 854
\end{tabular}

\section{Cherokee}

\begin{tabular}{ccccc} 
Group & Enrolled & Pretest & Intervention & Posttest \\
\hline 1 & 263 & 263 & 263 & 210 \\
2 & 277 & 277 & & 238 \\
3 & 218 & & 218 & 175 \\
4 & 238 & & & 192 \\
Total & 996 & 540 & 481 & 815 \\
\hline
\end{tabular}

ful use of lay health educators has been in providing outreach education. NCP is another example of the success of the lay health educator model. For Native American populations, the lay health educator approach seems particularly appropriate because of the complexities of the cultures involved. Community analyses conducted during NCP revealed that the Cherokee and Lumbee cultures are distinct from other ethnic cultures among the population of North Carolina as well as quite different from one another. Furthermore, the community analyses indicated that many differences are subtle. As a result, it seemed much more feasible to recruit, hire, and train Native American women who were part of these cultures themselves to conduct the education program than to train non-Natives about the Lumbee and Eastern Cherokee culture. Also, it was unclear whether Native American women would accept non-Natives as lay health educators, regardless of the training that could be provided by the research team. In essence, it was unlikely that training would make non-Native lay health educators acceptable to the target populations; however, Native American women who were already accepted could be trained to provide the health education intervention.

A similar situation was found for data collection. Recruitment, enrollment, and administration of the questionnaire required extensive communication with Eastern Cherokee and Lumbee men and women. The community analyses discovered that individuals who were not intimately acquainted with the target populations had difficulty communicating effectively. For example, "outsiders" who did not recognize prominent surnames in the population did not realize that inquiry about kinship could be used to develop rapport; thus, they were relatively unsuc cessful in their dealings with the target population. When local Native Americans were used. success in recruitment and administration increased dramatically. To a larger extent, the success of the project rested on the recruitment and training of Native Americans at the local level, supported by the project staff at WFUSM.

The behavioral results from the study and the proportion of women reporting Pap tests were somewhat surprising. During the course of the project, it 
became clear that the barriers to obtaining Pap tests were quite different for Eastern Cherokee and Lumbee women. Eastern Cherokee women were able to obtain services from the IHS facility at no cost, and the primary barriers that they faced were transportation and waiting time in the clinic. Lumbee women, on the other hand, had to identify a healthcare provider, seek services, and grapple with financial issues. Among Eastern Cherokee women, 73 percent who reccived the intervention reported having had a Pap test on the posttest, compared to about 74 percent of the Lumbee women. It was expected that more Cherokee women would have reported Pap tests because they could receive them at no cost. It is possible that this finding may reflect differences in the two cultures in attitudes toward healthcare providers and about healthcare in general or differences with respect to other factors not readily apparent. Additional research is needed to improve understanding of this issue.

Cultural appropriateness was clearly a key factor in the success of the intervention. By carefully analyzing the Native American culture, the investigators were able to integrate features into the intervention that facilitated its introduction to the study populations. Educational materials all featured Native American women and depicted situations that were both familiar and specific to the tribe and section of the United States. Again, the employment of Native American women to deliver the intervention increased cultural sensitivity by demonstrating to women that the intervention was especially for them.

The nature of the intervention itself also contributed to the results. The intervention was individualized and was driven by social learning theory. The intervention addressed the specific needs of women, the barriers that they had to overcome to obtain Pap tests, and the resources that they possessed and needed. In addition, one of the most prominent features used in the intervention was modeling of the desired behavior (going to a healthcare provider for a Pap test). The combination of attending to individual needs and demonstrating the specific behavior that was needed (to prevent premature death from cervical cancer) provided a powerful inducement to the women in the study.

In conclusion, NCP, a 5-year community-based cervical cancer prevention project, showed modest improvement in increasing the knowledge, intentions, and behaviors of Native American women to reduce cervical cancer risk. This project provided an opportunity to gain valuable insight into the effectiveness of a culturally sensitive lay health education program for Native American women of two distinct tribes in North Carolina. These data add to the current body of literature on the usefulness of the lay health educator in ethnic minority communities and provide an opportunity to examine the model in a previously ignored population at high risk for death from cervical cancer.

\section{REFERENCES}

1. Public Health Service. Indian Health Service. Trends in Indian health. Rockville, MD: U.S. Department of Health and Human Services; Public Health Service; Office of Planning, Evaluation, and Legislation; Division of Program Statistics, 1995.

2. Public Health Service. Indian Health Service. Regional differences in Indian health. Rockville, MD: U.S. Department of Health and Human Services; Public Health Service; Office of Planning, Evaluation, and Legislation; Division of Program Statistics, 1995.

3. Horner RD. Cancer mortality in Native Americans in North Carolina. Am J Public Health 1990;80:940-4.

4. Dignan $M$, Sharp $P$, Blinson $K$, Michielutte $R$, Konen J, Bell R, et al. Development of a cervical cancer education program for Native American women in North Carolina. J Cancer Educ 1995;9:235-42.

5. Michielutte R, Dignan MB, Wells IHB, Young LD, Jackson DS, Sharp PC. Development of a community cancer education program: the Forsyth County, NC, Cervical Cancer Prevention Project. Public Health Rep 1989;104:542-51.

6. Dignan M, Beal PF, Michielutte R, Sharp PC, Daniels LA, Young LD. Development of a direct education workshop for cervical cancer prevention in high risk women: the Forsyth County Project. J Cancer Educ 1990;5:217-23. 
7. Dignan MB, Michielutte R, Sharp P, Bahnson I, Young $L$, Beal $P$. The role of focus groups in health education for cervical cancer among minority women. I Community Ilealth 1990; 15:369-75.

8. Dignan M, Bahnsun I, Sharp P, Beal P, Smith M, Michielutte $R$, et al. Implementation of mass media community health education: the Forsyth County Cervical Cancer Prevention Project. Health Educ Res 1991;6:259-66.

9. Dignan M, Michielutte R, Blinson K, Sharp P, Wells HB, sands E. Cervical cancer prevention: an individualized approach. Alaska Med $1993 ; 35: 279-84$.

10. Rosenstock, IM. The health belief model and preventive health behavior. Health Educ Monogr 1974;2:354-86.

11. Bandura A. Social learning theory. Englewood Cliffs, NJ: Prentice-Hall, 1977.

12. Strecher VJ, DeVellis BM, Becker MH, Rosenstock IM. 'The role of self-efficacy in achieving health behavior change. Health Educ Q $1986 ; 13: 73-92$.

13. Green LW. Health education planning: a diagnostic approach. Palo Alto, CA: Mayfield, 1980.

14. Alcalay R. Rationale and guidelines for developing a minority health communication model. Unpublished manuscript for National Cancer Institute, 1980.

15. Welty TK. Finding the way: Indian-specific HRA released. IHS Primary Care Provider 1989; $14: 64-5$.

16. Campbell DT, Stanley JC. Experimental and quasi-experimental designs for research. Chicago: Rand McNally, 1963.

17. Dignan M, Michielutte R, Blinson K, Wells HB, Case I.D, Sharp P, et al. Effectiveness of health education to increase screening for cervical cancer among eastern-band Cherokee Indian women in North Carolina. I Natl Cancer Inst 1996;88:1670-6.

18. Dignan MB, Michielutte $R$, Wells HB, Sharp $P$, Blinson $K$, Case $R$, et al. Health education to increase screening for cervical cancer among Lumbee Indian women in North Carolina. Health Educ Res (in press).
19. Eng E, Parker E, Harlan C. Lay health advisor intervention strategies: a continuum from natural helping to paraprofessional helping. Health Educ Behav 1997:24:413-7.

\section{ACKNOWLEDGMENTS}

This project, "North Carolina Native American Cervical Cancer Prevention Project," was funded by the National Cancer Institute (Grant No. U01CA52256).

\section{AUTHORS}

Ronny A. Bell, Ph.D., M.S.

Section on Epidemiology

Department of Public Health Sciences

Wake Forest University School of Medicine

Medical Center Boulevard

Winston-Salem, NC 27157

Tel: (336) 716-9736

Fax: (336) 716-5425

E-mail: rbellarc phs.wfubmc.ectu

\section{Mark B. Dignan, Ph.D., M.P.H.}

AMC Cancer Research Center

1600 Pierce Street

Denver, CO 80214

Tel: (303) 23)-3363

Fax: (303) 233-1863

\section{Robert Michielutte, Ph.D.}

Department of Family and Community Medicine Wake Forest University School of Medicine Medical Center Boulevard

Winston-Salem, NC 27157

Tel: (336) 716 2241

Fax: (336) $716-3206$

E-mail: rmichiel@wfubmc.edu

\section{Penny Sharp, Ed.D.}

Department of Family and Community Medicine Wake Forest Lniversity School of Medicine Medical Center Boulevard

Winston-Salem, NC 27157

Tel: (336) 716-2129

Fax: (336) $716-3206$

E-mail: psharp@wfubmc.edu 


\section{Karen Blinson}

Department of Cardiology

Wake Forest University School of Medicine Medical Center Boulevard

Winston-Salem, NC 27157

Tel: (336) 716-6513

Fax: (336) 716-7500
John G. Spangler, M.D., M.P.H.

Department of Family and Community Medicine Wake Forest University School of Medicine Medical Center Boulevard

Winston-Salem, NC 27157

Tel: (336) 716-2248

Fax: (336) $716-3206$

E-mail: jspangle@wfubmc.edu 


\title{
Chapter 4 \\ Effects of a Culturally Tailored Intervention To Increase Breast and Cervical Cancer Screening in Native Hawaiians
}

Richard O. Banner, Carolyn Cook Gotay, Rachelle Enos, Doris Segal Matsunaga, Nancy Hedlund, Brian F. Issell, Ho'oipo DeCambra

\begin{abstract}
T his chapter describes the impact of a health education intervention to increase cervical and breast cancer screening among Native Hawaiians. The project drew on many aspects of the culture of the target population, both in the content and process of the group education and support intervention itself (known as a "kokua group") and in the development of a questionnaire to measure knowledge, attitudes, and behaviors of participants. A total of 340 women completed the questionnaire both before and after the kokua group. The women showed positive gains in knowledge after kokua group participation. With respect to behavioral intentions, we found significant changes for both Pap tests and mammograms: Women moved along the continuum of behavioral change toward the objective of getting regular Pap tests or mammograms. Increases in intentions to obtain the screening tests were likely influenced by gains in knowledge during the kokua group and also by increases in confidence to negotiate successfully in the health care system.
\end{abstract}

\section{INTRODUCTION}

The traditional Hawaiian value of kokua is defined as a mutual willingness to help, to help without an expectation of return and without having to be asked. This value provided a foundation for an intervention designed to increase breast and cervical cancer screening in a Native Hawaiian community. The intervention-referred to as a kokua group-consisted of education and support delivered by trained health educators in a group setting. The need for interventions such as kokua groups was based on cancer statistics, which indicated that Native Hawaiians are more than three times as likely as Hawaiian women of Japanese ancestry to die of breast cancer (1). This disparity is attributable, in part, to the fact that Native Hawaiian patients are diagnosed at a more advanced stage of disease than patients of other ethnicities (2). The overall objec- tive of the research project was to increase the use of cervical and breast screening in the community in an effort to contribute to reduction of disproportionate cancer mortality in this population.

The kokua group curriculum, like the project as a whole, was conducted through the cooperative efforts of community researchers, the project staff, and scientific researchers. The Wai anae Coast Comprehensive Health Center, a federally funded community health center, spearheaded the planning and conduct of the research. Community representatives, health professionals, and scientific researchers worked collaboratively to develop, implement, and evaluate the research project, which was known in the community as the Wai anae Cancer Research Project $(3,4)$.

This project attempted to address weaknesses of existing health promotion programs, which fre- 
quently have been poorly matched to the values, priorities, and preferred communication styles of minority communities (5). The curriculum incorporated traditional Hawaiian values such as aloba (love) and lokabi (harmony), in addition to kokua. The intervention focused on the group, as opposed to the individual, since the kokua groups built on preexisting social bonds among community members and stressed the importance of sharing knowledge with other women who did not participate in the intervention. Furthermore, the curriculum was designed to be flexible and to be delivered in the traditional "talk story" fashion. Rather than employing didactic presentation by the group leader, the sessions encouraged the sharing of individual experiences and feelings. This report provides a description of the development of a tool to measure the impact of the kokua groups, as well as an analysis of the effects of participation on women who took part. A one-group pretest-posttest design was used to examine changes in participants' knowledge, attitudes, and behavioral intentions. The target population was a community of about 45,000 , of whom approximately 18,000 were Native Hawaiians.

\section{METHODS}

\section{Overview of the Kokua Group Procedures}

The kokua groups were organized by bo okele (navigators), Native Hawaiian paraprofessional health educators from the community. These women served as the project staff, and the research team trained them in group facilitation techniques and provided them with current information on cancer and other health-related topics. The navigators' responsibilities were to recruit women to host the groups (Peer Group Leaders [PGLs]), conduct the groups, and follow up when women needed extra encouragement for screening or assistance for abnormal test results. The PGLs were women who were recognized as family or community leaders among their peers. Each PGL hosted one or more kokua group, inviting friends, family, and/or coworkers to attend. In most cases, the kokua groups were conducted in the PGLs' homes, although other locations (including the health center, project offices, and the beach) were used when necessary.

\section{Questionnaire To Measure Knowledge, Attitudes, and Behaviors}

\section{Content}

The research team developed a questionnaire primarily to evaluate the kokua group intervention. It was designed to measure short-term changes in participants' knowledge, attitudes, and behavioral intent regarding breast and cervical cancer screening. Participants completed the questionnaire before and after the kokua group. The self-administered questionnaire included questions about (1) demographic information; (2) social support for cancer screening; (3) knowledge, attitudes, behavior, and behavioral intent regarding cancer screening and treatment; and (4) awareness of and confidence in using health resources. The questionnaire also assessed knowledge of Hawaiian concepts and practices and satisfaction with the kokua group.

\section{Questionnaire Development}

During the initial pilot phase, some participants accepted and completed the questionnaires; however, some women voiced negative reactions, saying that the questionnaire reminded them of welfare forms or schoolwork. Some of the strongest negative reactions came from groups at a transitional "village" for homeless families. Navigators believed the questionnaire was generating resistance and making it harder for them to conduct the kokua group.

The project staff, along with community and scientific researchers, struggled with seemingly incompatible agendas of community and scientific standards. They went back to the drawing board and reconsidered other approaches. The research team redesigned the questionnaire to be more culturally acceptable while including items similar to those used in other screening studies.

The redesigned questionnaire was presented as a booklet entitled Ka Lei O Ike (KLOI) (the Garland of Knowledge), rather than as a standard form resembling a test. The KLOI booklet incorporated Hawaiian concepts, language, and images, starting with the metaphor of making a lei (garland). At the bottom of each page was a lei in the making. As respondents completed the questions on each page, another plant ingredient was added to the lei. When the final question was completed, the booklet pre- 
sented the following statement: "La haku ia oe $\mathrm{ika}$ lei o ike Mahalo nui loa." (You have completed the lei of knowledge. Thank you very much.) According to Hawaiian cultural expert Mililani Allen, "The concept of the lei was chosen because of the philosophy behind creating a lei, using the best materials and an attitude of love and caring so the le $i$ will be beautiful. It was also chosen because leis are a symbol of beloved persons in Hawaiian poetry."

The response options in the KLOI questionnaire included the Hawaiian language. Respondents checked 'Ae (yes), 'A'ole (no), or Paba (perhaps) to answer some questions. To provide a tie with traditional medicine, the back page had a listing of all plant ingredients used in the lei, along with their traditional uses. That page could be torn off by the respondent and taken home, providing the respondent with a concrete symbol of her participation.

The research team pretested the KLOI in two kokua groups where it was greeted enthusiastically, and virtually no refusals or negative feedback about the questionnaire were received during the remainder of the project.

\section{Administration of the KLOI}

The first administration of the KLOI took place after the bo'olauna (introductions of kokua group members) and a clescription of the research project. The introductions often were lengthy and involved sharing of health-related experiences. This part of the curriculum was vital to the effectiveness of the kokua group, creating the feeling of safety and comfort required for participants to open up and share experiences and information with each other and the project. Thus, the navigator administered the KLOI between 20 and 45 minutes after the formal start of the kokua group. While there was some risk that participants' answers may have been affected by the introductions, we believed adhering to cultural etiquette was critical. The navigator enhanced the acceptability of the KLOI by emphasizing that (1) it was a product developed by women in the community, (2) it would help the project team know whether they were doing a good job, (3) all answers were confidential, and (4) by filling out the questionnaire, the women would be contributing to the community research project and the health of Hawaiian women. For participants who were less comfortable with forms and questionnaires, the navigator read each question aloud, pausing for participants to write the answers in their booklet. Another staff member was sometimes present to offer assistance to individuals such as a nonreader, an elderly woman, or someone who forgot her glasses.

\section{Oversight of the Project}

The research team designed the structure of the project to maintain community ownership of the project. The Waianae Coast Comprehensive Health Center was the direct recipient of the grant funding, and its medical director served as the principal investigator, with co-principal investigators representing the community and the local cancer research center. The Steering Committee for the project was composed of these three individuals plus two additional community representatives and two additional scientists.

The research team chose community residents for a Community Advisory Committee on the basis of their involvement in community health activities, their knowledge of Hawaiian culture, and their understanding of the community. Kupuna (elders) were asked to participate because they are considered the keepers of cultural knowledge and wisdom. Community Advisory Committee members participated in a number of activities, depending on the needs of the project at any given time. Their volunteer involvement spanned 7 years: 2 years in proposal development and 5 years of project planning, implementation, and evaluation. In addition, professional project staff members, with experience in public health and community organizing, were essential to building and maintaining involvement and interest of these community consultants and maintaining liaison with the members of the Steering Committee and with the navigators. Three members of the health center staff were consistently involved over the entire course of the project. The stability of both staff and community involvement was a critical contribution to the project's success.

\section{RESULTS}

\section{Participation}

The KLOI proved to be culturally acceptable to most kokua group participants. Of the 500 kokua group 
participants, 470 contributed data by completing a KIOI before the group. The project goal of enrolling 500 participants in kokua groups was based on the primary study hypothesis: that women who participated in kokua groups would diffuse information about cancer screening to other women in the community, resulting in a community-wide change. The analysis of the impact of the intervention on the community is reported elsewhere $(\sigma)$. It was estimated that 500 participants in the intervention would result in measurable permeation within the community. A total of 340 women completed the questionnaire both before and after the kokua group. In virtually all cases, the absence of a post-KLOI was attributed to women who did not return for or complete the second group session; there was no evidence of any objection to completing the instrument.

\section{Description of Kokua Group Participants}

Table 1 shows a demographic profile of the 340 women who completed both questionnaires. The figures indicate that the kokua groups included a diverse group of women. A majority of the participants were under age 40 , were of Hawaiian ancestry, and had completed high school or higher schooling. Almost one-quarter of the women reported yearly family incomes of less than $\$ 10,000$, but almost all the women ( 96 percent) had some form of health insurance.

Before the kokua group, two-thirds of participants said that family members supported them in getting breast cancer screening and that they knew where to get information about cancer if they needed it. Somewhat more than half of the women followed the age-specific guidelines for mammograms and Pap smear tests: Women were considered adherent if they had had a Pap smear in the past year and if they had had a mammogram in the past 2 years for ages 40 to 49 or in the past year for ages 50 and older. (Kokua group recommendations that women age 40 and older obtain mammograms were the same throughout the period of the study, consistent with State policies for reimbursement of mammograms by health insurers.)

\section{Changes in Knowledge, Attitudes, and Behavioral Intentions}

The navigators asked the participants a series of questions about five risk factors for cervical and breast cancers. As shown in Table 2, the participants initially had little knowledge about these risk factors. The only item for which more than one-third of participants answered correctly pertained to familial breast cancer. After kokua group participation, the women showed substantial gains in knowledge $\left(\mathrm{X}^{2}\right.$ $<.001$ by McNemar's test for all items).

Participants were also asked four questions related to attitudes about cancer. Table 2 shows that the women's attitudes toward cancer showed positive change in some areas and no change or negative change in others. After participating in a kokua group, significantly more participants agreed that cancer of the cervix can be cured if found early and that cancer treatment was worth getting if it would help them to live longer ( $\mathrm{X}^{2}<.001$ by McNemar's test). However, no change was seen between pretest and posttest in agreement with these statements: "I'm too busy taking care of my family to get Pap smears and breast exams," and "If I had cancer, I would want to know."

With respect to behavioral intentions, participants were asked to choose a statement that best described their plans to obtain screening in the future (Table 3). These statements were constructed in concordance with the "Stages of Change" model, which has heen used to describe the process that individuals go through in adopting health-related behaviors (7). Before the kokua group, with respect to having a Pap test, 22 percent of the women said they "were not even thinking about it," "need to think about it," or "had one before but were not thinking about getting another." The post-kokua group indicated that only 8 percent of women responded similarly. Twenty-nine percent of the women reported that they were planning to make an appointment (compared to 19 percent pre-kokua group), and 64 percent said they were in adherence (compared to 59 percent pre-kokua group). This contrast was significant $(\mathrm{z}=-5.45, \mathrm{p}<.001$ by Wilcoxon signed rank test).

A similar trend was scen with mammograms for women age 40 or older (Table 3). Before the kokua group, 16 percent of the women said they "were not even thinking about it," "need to think about it," or "had one before but were not thinking about getting another"; afterwards, 8 percent responded in these categories. The proportion of women who said they were planning to make an appointment and that 


\section{TABLE I: Description of Kokua Group Participants ${ }^{a}$}

$\begin{array}{lll}\text { Age }^{\mathbf{b}} & \text { Under } 40 & 55 \% \\ & 40 \text { to } 59 & 33 \\ & 60+ & 12 \\ \text { Ethnicity }^{\mathbf{b}} & & 57 \% \\ & \text { Hawaiian/part Hawaiian } & 43 \\ & \text { Not Hawaiian } & 12 \% \\ \text { Formal Western Education }^{\mathbf{b}} & \text { Less than High School } & 49 \\ & \text { HS graduate or GED } & 39 \\ & \text { Education past HS } & 23 \% \\ \text { Family Annual Income }^{\mathbf{b}} & <\$ 10,000 & 32 \\ & \$ 10-25,000 & 15 \\ & \$ 25-35,000 & 31\end{array}$

Medical Insurance ${ }^{b}$

Social Support

Screening Behavior ${ }^{c}$
None $\quad 4 \%$

Medicaid/Medicare/SHIP ${ }^{d}$

Private $\quad 62$

Other 9

Family encourages screening $\quad 68 \%$

Know where to get cancer information 66

Have people to talk to about feelings about cancer 74

Ever had a Pap smear $\quad 83 \%$

Pap smear within past year 64

Ever had a mammogramb

Had mammogram according to guidelines ${ }^{b}$

a Based on women who completed questionnaires before and after kokua group participation $(n=340$; responses are lower on individual questions due to nonresponse). Numbers represent percentage in each category.

b Numbers represent percentage of total sample.

c Numbers represent percentage responding affirmatively to each question. Women 40 years and older only: $n=208$.

¿ State Health Insurance Plan.

they were getting mammograms also regularly increased after the kokua group. This contrast was significant $(z=-2.44, p=.015$ by Wilcoxon signed rank test). 
TAELE 2: Knowedge and Attitudes Before and After Kokua Group Participation

Variable

Percentage of Yes Responses

\section{Knowledge}

Hawaiian women more likely to die from breast cancer.

A woman is more likcly to get breast cancer if . . .

... her mother or sister had it.

... she eats a diet high in fat.

A woman is more likely to get cervical cancer if . . .

... she began having sex early and had many partners.

31

92

... she smokes cigarettes.

87

\section{Attitudes and Beliefs}

If cervical cancer is found early, it can be cured.

Cancer treatment . . . worth getting if it helps me live longer.

61

I'm too busy taking care of family to get a Pap/breast exam.

67

67

If I had cancer, I would want to know.

67

"Based on uomen who completed questionnaires before and after kokua group participation ( $n=340 ;$ responses are lower on individual questions due to nonresponse). Numbers represent percentage of women wbo answered "yes" to each question. The remaining women said "no" or "perbaps."

" McNemar chi-square values were significant $(p<.001$ ) for first seven questions; chi-square values were 160.2, 48.49, $176.4,183.0,295.2,50.3$, and 63.7, respectively. Responses on the last tuo questions did not differ significantly.

\section{Changes in Healthcare Confidence}

To determine how the kokua groups may have affected behavioral intentions, we compared the women's confidence in their ability to obtain healthcare before and after the kokua group in two groups: those who had a history of cancer screening and those who did not. Women who had received a Pap test within the past year were compared with women who had not, and women age 40 or older who had ever had a mammogram were compared with those who had never had one.

The women were asked to indicate their agrcement with statements about five aspects of healthcare, all of which were addressed in the kokua group curriculum. They responded "yes," "no," or "perhaps" to statements such as, "I will be able to ask the doctor or nurse any questions I have." Table 4, which compares changes in conficlence before and after kokua group participation, shows that before the kokua group, the women who had not had screening were less likely to endorse all items; that is, they were less likely to feel that they could ask questions, that they could talk about their feelings, that they would be treated with respect, that they could complain, and that they would be able to pay for the services. The same trends were observed for Pap testing and for mammography.

After the kokua group, there were considerable gains in confictence in all areas and among both groups of women. Virtually all women felt confident that they would be able to ask questions, express their feelings, be treated with respect, and complain. Responses were virtually identical in the two groups. With respect to being able to pay for care, there were large gains in both groups, although the women who had not used scrcening in the past 
TABLE 3: Changes in Behavioral Intent Before and After Kokua Group Participation ${ }^{a}$

Variable

Percentage of Yes Responses

Variable

Pap Smears $(n=336)$

Getting pap smears regularly

Planning to make an appointment

Had one before but not thinking about getting another

Need to think about getting one

Have not even thought about getting one
$59 \%$

19

Before After

10

8

4

$64 \%$

29

5

2

1

\section{Mammography $(n=148)^{c}$}

Getting mammograms regularly

$60 \quad 63$

Planning to make an appointment

Had one before but not thinking about getting another

Need to think about getting one

$24 \quad 30$

$3 \quad 6$

Have not even thought about getting one

7

6

a Based on women who completed questionnaires before and after kokua group participation. Numbers represent percentage in each category.

b Wilcoxon signed rank tests indicated significant differences before and after group participation for mammography $(z=-2.44, p=.015)$ and Pap smears $(z=-5.45, p<.001)$, comparing women getting tests regularly, women planning to make an appointment, and women in the other three response categories combined.

Women 40 years and older only.

were still somewhat less likely to believe they could pay for care. An analysis of health insurance coverage in the overall sample of kokua group participants $(n=470)$ indicated that women who did not have regular Pap tests were four times as likely $(8$ percent vs. 2 percent) to be without health insurance as women who received annual Pap tests $\left(\mathrm{X}^{2}(1)=8.24, \mathrm{p}<.01\right)$.

\section{DISCUSSION}

This project developed and used a health education intervention to increase cancer screening that drew on many aspects of the culture of the target population to be successful. The intervention included Hawaiian cultural themes in its content and presentation. While the importance of cultural tailoring was obvious from the beginning, the need to adapt assessment strategies did not become apparent until pilot- testing. The research team expended considerable time and effort in designing a questionnaire that would be acceptable to kokua group participants.

The research team found the questionnaire, the $\mathrm{Ka} \mathrm{Lei} \mathrm{O}$ Tke, to be an acceptable and useful instrument to use before and after the kokua groups. The questionnaire incorporated local art and jargon, and it communicated an understanding and respect for traditional Hawaiian culture and practice. In fact, the KLOI became more than an evaluation method. It became a teaching tool for the navigators, who found that the initial administration of the KLOI sparked questions, whereas the second administration after the kokua group offered a useful review of key messages. The KLOI reinforced the messages about the vital link between health and Hawaiian values, and it demonstrated the theme of reciprocity, becoming the first "gift" to and from the project. 
TABLE 4: Changes in Confidence in Health Care Before and After Kokua Group Participation: Women Who Are Receiving Screening vs. Women Who Are Not

Pap Test $(N=340)^{a}$

Mammogram $(N=148)^{b}$

Variable

Annual Pap (\%)

$(N=173)$

$>1 \underset{(N . P a p(\%)}{(N=164)}$

Mam ever (\%)

$(N=109)$

No $\operatorname{Mam}(\%)$

$(N=31)$

Will Be Able To Ask Questions

$\begin{array}{llllr}\text { Before } & 91 \% & 86 \% & 91 \% & 79 \% \\ \text { After } & 99 & 96 & 99 & 100\end{array}$

Will Be Able To Discuss True Feelings

$\begin{array}{lllll}\text { Before } & 88 & 74 & 88 & 69 \\ \text { After } & 97 & 94 & 98 & 97\end{array}$

Will Be Treated With Respect

$\begin{array}{lllll}\text { Before } & 91 & 67 & 86 & 73 \\ \text { After } & 95 & 93 & 95 & 94\end{array}$

Will Be Able To Complain

$\begin{array}{lllll}\text { Before } & 87 & 78 & 89 & 82 \\ \text { After } & 96 & 98 & 99 & 95\end{array}$

Will Be Able To Pay

$\begin{array}{lllll}\text { Before } & 71 & 57 & 74 & 55 \\ \text { After } & 85 & 79 & 92 & 78\end{array}$

a Based on women who completed questionnaires before and after kokua group participation ( $n=340$; responses are lower on individual questions due to nonresponse). Numbers represent percentage of women who answered "yes" to each question. The remaining women said "no" or "perbaps."

b Women 40 years and older only.

According to Community Advisory Committee members, income levels and the extent of formal Western education of kokua group participants were fairly comparable to that of the community. The percentage of kokua group participants with no health insurance ( 4 percent) is comparable to estimates for the State of Hawaii as a whole. The rate is probably higher in the Wai anae Coast area, although no estimates are available specific to this area.

The project made special efforts to reach Native Hawaiian women age 40 and older for whom mes- sages about early detection of breast cancer are most appropriate. Comparative data indicate that the project was successful in reaching these target populations. While no definitive figure is available, it is estimated that Native Hawaiians constitute between 38 percent and 54 percent $(8)$ of the Wai anae Coast population. Fifty-seven percent of kokua group participants were Hawaiian or part-Hawaiian. Furthermore, kokua groups included a higher percentage of women over 40 than the very young Wai anae Coast population: While 20 percent of the Wai anae Coast population 
are estimated to be over 40 years of age (9), 45 percent of kokua group participants were 40 or older.

The percentages of participants reporting mammogram screening and annual Pap smears were quite close to the rates found in the community-wide baseline telephone survey (3). However, 18 percent of kokua group participants reported they had never had a Pap smear, whereas the comparable percentage in the baseline community-wide survey was only 6 percent (3). This finding indicates that the kokua groups reached women in particular need of education and advice about screening. Thus, the kokua groups did not only "preach to the choir" of women who already understood the importance of screcning, they actually reached a group who did not.

It should be mentioned, however, that only 340 out of 470 ( 72 percent) of the women who completed the first $\mathrm{Ka}$ Lei O Tke completed a second questionnaire. According to the navigators, this occurred generally because they were absent from the second session of the kokua group. An analysis of demographic and behavioral characteristics of women who completed both questionnaires compared with those who did not revealed a number of differences. Women who attended only one session were older $\left(\mathrm{X}^{2}(2)=8.9, \mathrm{p}=.012\right)$ and had less formal Western education $\left(\mathrm{X}^{2}(2)=25.2, \mathrm{p}<.001\right)$ than women who attended both sessions. They were also less likely to have ever had a Pap smear ( 77 percent vs. 83 percent, $\mathrm{X}^{2}(1)=5.2, \mathrm{p}=.023$ ) or a mammogram $\left(63\right.$ percent vs. 76 percent, $\mathrm{X}^{2}(1)=6.3, \mathrm{p}=$ .012). The differences indicate that the findings about changes attributable to group participation should be restricted to women who are willing to complete a health intervention such as the kokua group curriculum. It may be that different approaches to screening programs (e.g., briefer interventions, actual visits to a clinic) are required if we are to reach older women with limited educational resources and those who have had little or no previous experience with screening.

Most women came into the group reporting high levels of social support: More than two-thirds indicated that their family members encouraged them to get screening, and three-quarters said that they had people to talk to regarding their feelings about cancer. This finding may reflect the importance of social ties in Hawaiian culture. These perceptions may also have been influenced by the intervention-by hav- ing been invited to the kokua group by someone in their social network; therefore, they may not be a true preintervention measurement.

The KLOI results indicated that the kokua group had a strong impact on knowledge, attitudes, and behaviors. With respect to knowledge, there were increases in the number of correct responses for all questions. Attitudinal items showed more variation. The pattern of positive changes in some items but not in others implies that participants tended to respond honestly, not giving only "socially desirable" answers. Community investigators and the project staff interpreted these data as reflecting realistic ambiguity and fears and social obligations, but not necessarily barriers to actual participation in screening. For example, women were heard to remark, "Yes, l'm too busy taking care of my family to get this screening, but I'm going to do it anyway," and "I wouldn't want to know I had cancer, but if I knew I'd get it treated."

With respect to behavioral intentions, significant changes were found for both Pap tests and mammograms: Women moved along the continuum of behavioral change toward the objective of getting regular Pap tests or mammograms. Increases in intentions to obtain the screening tests were likely influenced by gains in knowledge during the kokua group and by increases in confidence about negotiating successfully in the healthcare system. This was dramatically demonstrated in the contrast between women who were receiving regular screcning before the kokua group and those who were not. Those getting regular screening came into the kokua groups with more confidence that they could find health information and communicate with medical providers. Those not getting regular screening gained in consumer confidence during the kokua group, ending with the same level of confidence as the initially more adherent women. For example, women who had never had a mammogram were less likely to think they would be able to ask a doctor questions before the kokua group, but just as likely to think they would be able to ask questions after participating in a kokua group. The same was true for questions concerning "talking to the doctor about my true feelings and concerns" and "being treated with respect." The same pattern held for Pap tests: The lower levels of confidence about interactions with the healthcare system among women who 
were not adhering to Pap screening guidelines at the pretest had disappeared by the time of the posttest.

How long this enthusiasm was maintained and whether it translated ultimately into short- or longterm screening adherence are not known. However, the kokua groups appeared to create a sense of empowerment among participants. Gains in knowledge and positive behavior intentions were demonstrated, even among women who had never before participated in cancer screening. The social support among the attendees and cultural values that were incorporated into the kokua group curriculum and the $\mathrm{Ka}$ Lei O Ike questionnaire contributed to a greater willingness to participate in cancer screening and enhanced self-confidence in dealing with healthcare systems.

In conclusion, the project tested the effects of a culturally tailored intervention called a kokua group in increasing breast and cervical screening in Native Hawaiian women. The research team developed, implemented, and analyzed the project with the full participation of the community. Kokua group participants demonstrated significant positive changes in knowledge, attitudes, and behavioral intentions after the group sessions, indicating the effectiveness of this intervention in encouraging cancer screening among women who took part. The culturally tailored approach. anchored in the mores of the community in which it is based, offers the prospect of reduced mortality from breast and cervical cancers among Native Hawaiian women.

\section{REFERENCES}

1. Hawai i Tumor Registry data, 1995.

2. LeMarchand L, Kolonel L. Cancer: epidemiology and prevention. The health of Native Hawaiians: a selective report on health status and health care in the 1980s. In: Wegner E, editor. Social process in Hawaii. Vol. 32. Honolulu: University of Hawaii Press, 1989:134-48.

3. Matsunaga DS, Enos R, Gotay CC, Banner RO, DeCambra $\mathrm{H}$, Hammond OW, et al. Participatory research in a Native Hawaiian community: the Wailanae Cancer Research Project. Cancer 1996;78(7 Suppl):1582-6.

4. Banner RO, DeCambra H, Fnos R, Gotay C, Hammond $O W$, Hedlund $N$, et al. A breast and cervical cancer project in a Native Hawaiian community: the Wai anae Cancer Research Project. Prev Med 1995;24:447-53.

5. Balshem M. Cancer, control, and causality: talking about cancer in a working class community. Am Ethnol 1991:18:152-70.

6. Gotay CC, Banner RO, Matsunaga DS, Hedlund $N$, Enos R, Issell BF, et al. The impact of a culturally appropriate intervention to increase breast and cervical screening in Native Hawaiian women. Unpublished manuscript, Cancer Research Center Hawaii and Liniversity of Hawaii, Honolulu, 1998.

7. Prochaska JO, velicer WF, Rossi JS, Goldstein MG, Marcus BH, Rakowski W, et al. Stages of changes and decisional balance for 12 problem behaviors. Health Psychol 1994;13:39-46.

8. U.S. Bureau of the Census, 1990. Census of the population: general population characteristics. Washington, DC: U.S. Department of Commerce, 1992.

9. Department of Health. Health Surveillance Survey, State of Itawaii. Honolulu: State of Hawaii, Department of Health, 1989.

\section{ACKNOWLEDGMENTS}

Special appreciation is extended to Judy Cocquio, Nita Ilaban, Nalani Tavares. Rell Sunn, Mililani Allen, and members of the Community Advisory Committee of the Wai anae Coast Cancer Research Project, as well as to the women who gave so generously of their time to take part in this project. We also are grateful for the scientific contributions of JoAnn Tsark and Ormond Hammond and for the assistance of Jeffrey D. Stern and Miles Muraoka in data analysis. This research was supported by a grant from the National Cancer Institute (U01CA52237).

\section{AUTHORS}

\section{Richard O. Banner, M.D.}

Wai anae Coast Comprehensive Health Center

Wai anae, HI 96792

Tel: (808) 696-1599

Fax: (808) 696-1533

E-mail: rio.banner@mcsx.com 


\section{Carolyn Cook Gotay, Ph.D.}

University of Hawai i

Cancer Research Center of Hawai $i$

1236 Lauhala Street

Honolulu, HI 96813

Tel: (808) 586-2975

Fax: (808) 586-3016

E-mail: cgotay@crch.hawaii.edu

\section{Rachelle Enos, M.P.H.}

Wai anae Coast Comprehensive Health Center

Wai anae, HI 96792

Tel: (808) 696-1599

Fax: (808) 696-1533

\section{Doris Segal Matsunaga, M.P.H.}

Wai anae Coast Comprehensive Health Center

Wai anae, HI 96792

Tel: (808) 696-1599

Fax: (808) 696-1533

E-mail: 75132.3074@compuserve.com

\section{Nancy Hedlund, Ph.D.}

University of Hawai $i$

Cancer Research Center of Hawaì $i$

1236 Lauhala Street

Honolulu, HI 96813

Tel: (808) 586-2975

Fax: (808) 586-3016

E-mail: hedlundn@juno.com

\section{Brian F. Issell, M.D.}

University of Hawai i

Cancer Research Center of Hawaì $\mathrm{i}$

1236 Lauhala Street

Honolulu, HI 96813

Tel: (808) 586-2975

Fax: (808) 586-3016

E-mail: brian@crch.hawaii.edu

\section{Ho'oipo DeCambra}

Wai anae Coast Comprehensive Health Center Wai anae, HI 96792

Tel: (808) 696-1599

Fax: (808) 696-1533 


\title{
Chapter 5 \\ Enhancing Tobacco Control Policies in Northwest Indian Tribes
}

\author{
Edward Lichtenstein and Kerri Lopez
}

\section{ABSTRACT}

consultation intervention to improve policies for control of tobacco use in Northwest
Indian tribes was developed and evaluated. The intervention included tribal representa-
tives attending a kickoff workshop, distribution of tobacco policy workbooks, followup visits to the tribes, and telephone consultation. Policy status and strength were assessed through telephone interviews with key tribal contacts and through review of policies approved by tribal councils. Two independent rounds of intervention led to significant pre- to postimprovements in tobacco use policies. The project was a collaborative effort between research/academic institutions and the Northwest Portland Area Indian Health Board (NPAIHB). Much of the project's success was attributable to the role of the Board in both planning and implementing the project. The policy change approach takes advantage of the structure and political independence of Indian tribes and may be applicable to reduction of other risk factors. The project used a qualitative approach to report its findings.

\section{INTRODUCTION}

Diseases related to smoking are a major cause of death among Native Amcrican people (1). Although smoking has generally declined over the past 20 years in the United States, smoking rates among Indian people, particularly women (2), remain high (3-4). Among Northwest Indians, rates of both smoking and smokeless tobacco use are at least 50 percent higher than those of the region's general population (5). Health problems are a concern not only for smokers; nonsmokers exposed to tobacco smoke also can be affected (6-8), making tobacco control among Indian people even more important.

One strategy to reduce exposure to tobacco smoke is to help individuals change their behavior; one example of this is the use of smoking cessation programs (9). A different approach is to change the environment around individuals to alter individual smoking behavior and to help protect individuals from tobacco smoke (10). Adopting a strong tobacco control policy (11) is an example of this approach. We developed and evaluated a program to enhance the tobacco control policies of Northwest Indian tribes. This paper describes how the work was done, summarizes the major findings, and discusses their implications. More technical detail about the project has been published elsewhere (12-17).

In recent years, the number and strength of policies to restrict tobacco use have increased in settings outside tribal communities $(18,19)$. These policies can reduce environmental smoke exposure, enhance participation in smoking cessation programs, and establish nonuse of tobacco as the norm, making it less likely that young people will use tobacco. Indian Health Service facilities became smoke-free in 1987 (20), but little information is available about smoking policies in other Indian 
contexts. In 1989, NPAIHB, along with researchers at the Oregon Research Institute (ORI) and the Oregon State University (OSU) Department of Anthropology, started the Tribal Tobacco Policy Project. NPAIHB was established in 1972 to provide consulting and educational services to the federally recognized tribes in the Pacific Northwest (Washington, Idaho, and Oregon). All tribes participating in the project have elected representatives on the Board.

At the first meeting, researchers and tribal representatives focused on an educational project aimed at tobacco cessation. As ORI and OSU scientists learned more about the geographic location and political organization of the Indian tribes, they found problems with this type of approach. Many of the Northwest tribes were small and far away from the paricipating organizations, which would make individual smoking cessation programs difficult to manage. Also, for research purposes, it would be desirable to randomly assign half of the individual smokers within a tribe to a cessation program and to leave the other half out; this would be difficult both culturally and practically. Instead, it made sense to try a program encouraging tribes to adopt strong tobacco control policies. Each tribe was politically and structurally autonomous, with control over settings, such as buildings, and tribal employees, making such a program possible and attractive. Tribes that adopted new tobacco use policies or strengthened established policies could heighten awareness of tobacco as a public health issue and pave the way for later educational programs. We hypothesized that a tobacco policy consulting program, spurred and supported by the NPAIHB staff, would significantly improve tribal policies on tobacco use.

All three organizations-NPAIHB, ORI, and OSU-worked to develop the proposal that led to funding for the project. NPAIHB passed a resolution approving the concept of the project, a required procedure for Board projects, and obtained letters of support from many of the tribes. Research design was a key issue. The ORI researchers, experienced with scientific procedures, wanted to randomly assign half the tribes to receive the tobacco policy consulting help and the remaining half to receive no consulting advice. The NPAIHB staff, obliged to serve the tribes represented by the Board, wanted to provide the consulting help to all tribes. A compro- mise presented a good solution: Half the tribes would receive the consulting help immediately, and the other half were assigned to a waiting list to receive consulting later. This provided a scientific evaluation of the program and enabled us to provide services to all interested tribes served by the NPAIHB. With this understanding, tribes were cooperative and agreed to be randomly assigned to either immediate consulting or a waiting list to meet research gorals. This plan had the added advantage of allowing us to revise the consulting program on the basis of experience with the first set of tribes.

\section{METHODS}

Thirty-nine autonomous Indian tribes in the Pacific Northwest participated in the project. The tribes varied greatly in size, culture (coastal, Great Basin, or plateau), and economy. Near the beginning of the project, we learned that 25 tribes (64 percent) received income from tobacco and 22 tribes $(56$ percent) had either lenient smoking policies or no such policies. Tobacco use policies in these tribes were assessed three times: at the beginning of the program in the spring of 1991, hefore the first group of tribes received consulting help; from January through March 1993, before the second group of tribes received consulting on their tobacco policies; and finally, in July through September 1994.

\section{Outcome and Policy Measurement}

To assess tribal policies on tobacco use, a standard ized telephone interview was done with two key members of each tribe who knew about tribal tobacco policies and health promotion activities. Key tribal contacts included health directors and general managers. Telephone interviews were used to save travel time and costs. Key contacts were used, rather than regular tribal members, because our focus was on the policy itself, not on how it was perceived by tribal members. The telephone interviewer was an Indian staff member of NPAIHB who was not connected with the consulting program. Details of the interview, procedures for resolving differences between the two representatives, and agreement among contacts have been presented elsewhere (13,14).

Policies were classified as smoke-free, moderately restrictive, or lenient/no policy within each of three areas common to all tribes: tribal council 
meetings, tribal work areas, and private offices. Lenient policies were scoted zero, moderately restrictive policies were scored one, and smoke-free policies were scored two within each area. A total score was computed as the sum of the three area scores for each tribe. In addition, tribes were designated as either being smoke-free or not smoke-free in all three areas.

Records were kept, including a checklist completed by the program staff, to provide information on tobacco use policies and the approximate date they were passed or revised. The checklist also documented how the policy consultation was done and listed the number of visits and telephone calls to each tribe.

\section{Obtaining and Maintaining Tribal Cooperation}

After Federal funding was secured, several steps were taken to obtain cooperation from the tribes. The project was described in NPAIHB's quarterly newsletter, discussed at quarterly Board meetings, and outlined in letters to key tribal contacts, including chairs of health committees and general managets. The project staff worked hard to schedule presentations about the project at all 39 tribal council meetings. Presentations included an overview of the project and its rationale. These efforts increased tribes' awareness of the project and tobacco policy issues and set the stage for the consulting work to come.

Indian professional staff members helped to develop the tobacco policy consulting programs. They carried out the program and kept records to help evaluate its success. For example, NPAIHB field directors helped to write the Tribal Tobacco Policy Workbook, and an Indian contractor contributed the artwork. The consulting and evaluation staff always identified themselves as NPAIHB employees when dealing with tribal members. This bolstered perceptions that the project was at least partly "Indianowned." The researchers assumed that our Indian colleagues would know best how to work with the tribes in a culturally sensitive way. It was important for the NPAIHB staff to carcfully distinguish between traditional use of tobacco and recreational, habitual misuse. Project implementation and evaluation details were negotiated at regular meetings, which rotated among the three participating settings.

\section{Consultation Process}

The consulting plan was originally designed by NPAIHB and research institution staff members, then revised in light of experience with the first group of tribes (14). The plan included some standard procedures, but it gave individual tribes flexibility to decide how to strengthen their tobacco control policies. Each tribe received a tobacco policy workbook that explained the importance of tobacco use policies; gave guidelines for choosing, developing, and implementing a policy; and included some examples of policies. Tribes were invited to send representatives to a kickoff meeting that was held concurrently with a quarterly Board meeting of NPAIHB (at which all tribes were represented). There was a half-day workshop with an overview of the project, a summary of the health risks of smoking and exposure to "second-hand" smoke, a viewing of the video On the Air, and an introduction to the Tribal Tobacco Policy Workbook.

After the workshop, the consulting program began with visits to the tribe by a project staff member, telephone calls, and intermittent contact by mail. Because the tribes differed in size and decisionmaking styles, we were flexible about the number of visits or contacts. With larger tribes, the project staff often worked with a tribal health committee. With smaller tribes, the staff usually worked directly with tribal councils. Visits were timed so that the project staff could meet with a health committee or tribal council.

When meeting with tribal groups, the project staff typically began by briefly describing the value of a tobacco use policy, which may protect youth and nonsmokers from tobacco smoke. To bring the tobacco and health issues closer to home, tribal members were asked to share their views about tobacco use, such as youth smoking and chewing. When a health committee or tribal group was interested in developing or revising a policy, the staff often showed sample policies that could be tailored to create a policy that the tribe's members would accept. The most sensitive issues were usually the strength of the policy (the range of settings where smoking would not be allowed) and enforcement of the policy (who was responsible for enforcing it and how people who violated the policy would be punished). One staff member 
sometimes used her laptop computer to modify a policy on the spot after discussion with tribal representatives; she then left a printed copy for the tribal council to consider.

As the project staff came across tribal members at quarterly Board meetings or in other Board projects, they often asked about the tobacco policy and made suggestions. Tribes that wanted information on tobacco cessation were given Indianspecific materials, such as self-help pamphlets featuring Indian models and topics developed by another Indian organization. Project staff members distributed antitobacco posters when visiting the tribes.

Our goal was to have each tribal council approve a new or improved tobacco use policy. Tribes could choose not to consider or approve a policy, or to retain the current policy.

\section{RESULTS}

\section{Implementation}

Table 1 summarizes the features of the tobacco policy consulting program. Seventy-five percent of the tribes sent representatives to the kickoff meeting. On average, project staff members visited tribes twice. Many phone calls were made as part of the program. During these usually brief calls, the staff supported progress and helped solve problems that hampered policy development.

\section{Policy Outcomes}

Figure 1 shows significant tobacco policy changes for both the tribes that received consulting help first and those that received consulting help later. Policies were stronger, and more tribes were smokefree in all three common areas by the end of the program. The number of tribes having smoke-free policies in all three common areas more than doubled in both groups of tribes.

Program records, especially checklists kept by project staff, documented tobacco use policies approved by tribal councils and their approximate dates of approval. This information is displayed in Figure 2. Several tribes enacted policies before the start of this project, but many more enacted policies cluring the consulting process, which took place from 1991 to 1992 for the first group of tribes and from 1993 to 1994 for the second group.

\section{DISCUSSION}

A consulting program using a specially developed workbook significantly increased the number of Northwest Indian tribes with strong tobacco control policies. Improvement in tobacco use policies was consistent across both groups of tribes, reflected both in interviews with key tribal representatives and in program records of enacted policies. The program was equally successful with the first and second groups of tribes even with a change of field directors, which illustrates the effectiveness of the program.

\section{TABLE 1: Activities for Intervention Tribes: Proportion or Median}

Percentage
of Tribes
Represented
at Kickoff
Meeting

Number of Planning Meetings (Average)

$\begin{array}{cc} & \text { Number of } \\ \text { Number of } & \text { Other } \\ \text { Phone Calls } & \text { Contacts } \\ \text { (Average) } & \text { (Average) }\end{array}$

Weeks of Consulting Help (Average)

\section{First group-}

20 Tribes

$75 \%$

2

9.5

1.3

45

\section{Second group-}

19 Tribes

$74 \%$

2

6.0

$N A^{b}$

36

" Brief meetings at other Board events that included mention of tobacco policy.

"Information not recorded during this phase of project. 
FIGURE I: Telephone Interview Tobacco Policy Scores Before and After Tribes Received Consultation

\section{Tobacco Policy Strength (Range $=0$ to 6 )}

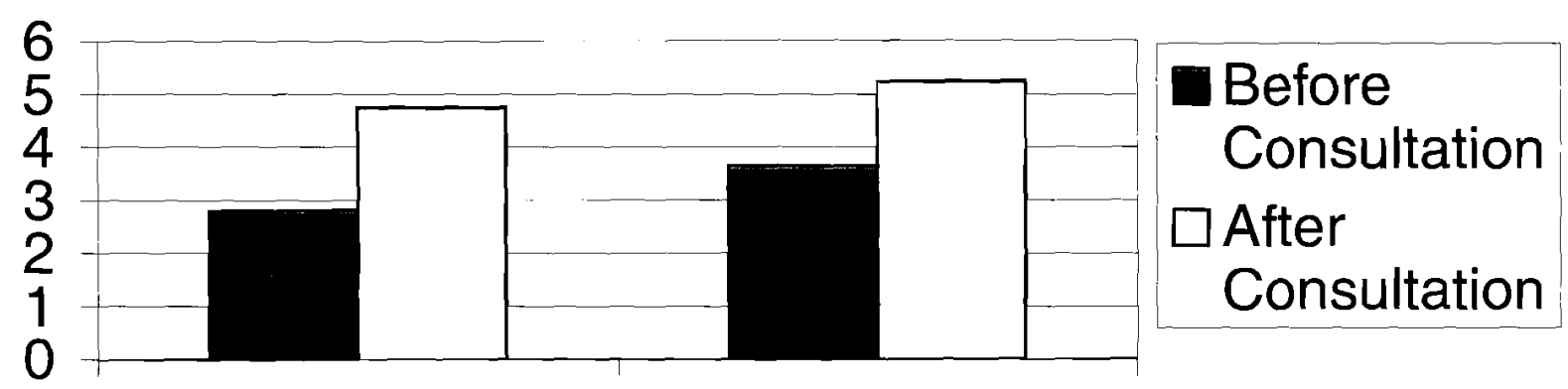

First Group of Second Group of Tribes Tribes

\section{\% Tribes Smoke-free in All Three Primary Areas}

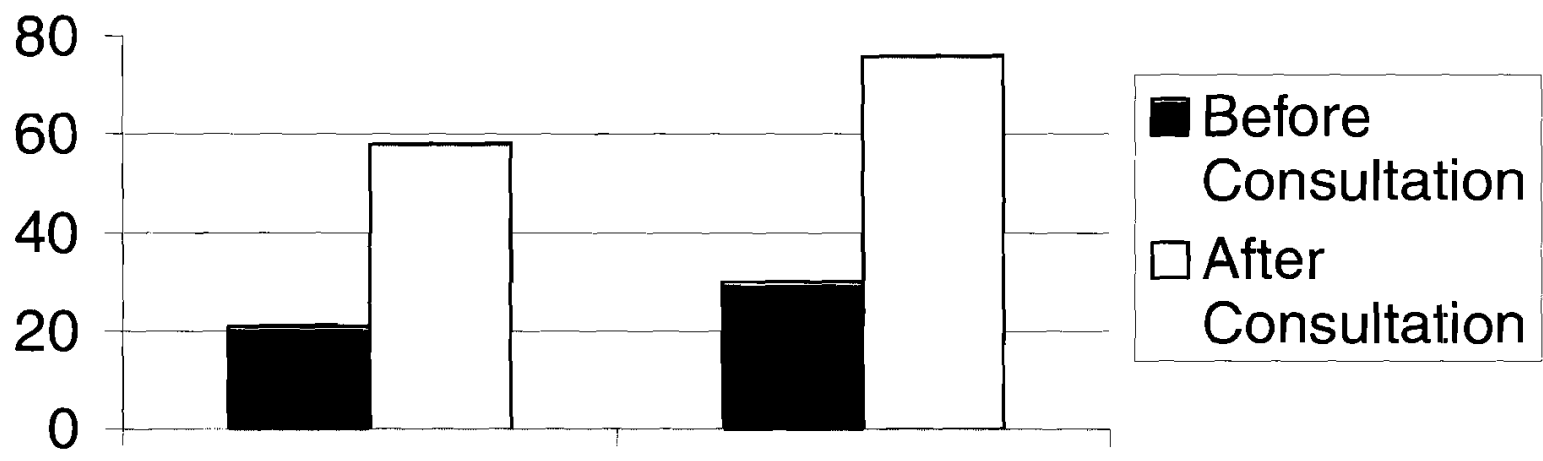

First Group of Second Group of Tribes Tribes

The consulting process was also fairly consistent across both groups of tribes. In both, about three-fourths of the tribes sent a representative to an orientation meeting, and the staff recorded an average of two visits or face-to-face meetings with each tribe. Consulting with the second group of tribes took somewhat longer (an average of 45 wecks rather than 36 weeks) and involved more phone conversations ( 9.5 rather than 6.0 , on average) because of a collective decision to try more options before ending the program with any tribe. 
FGURE 2: Tribal Counci-Approved Yobacco Policies, by Year, for the Two Groups of Tribes

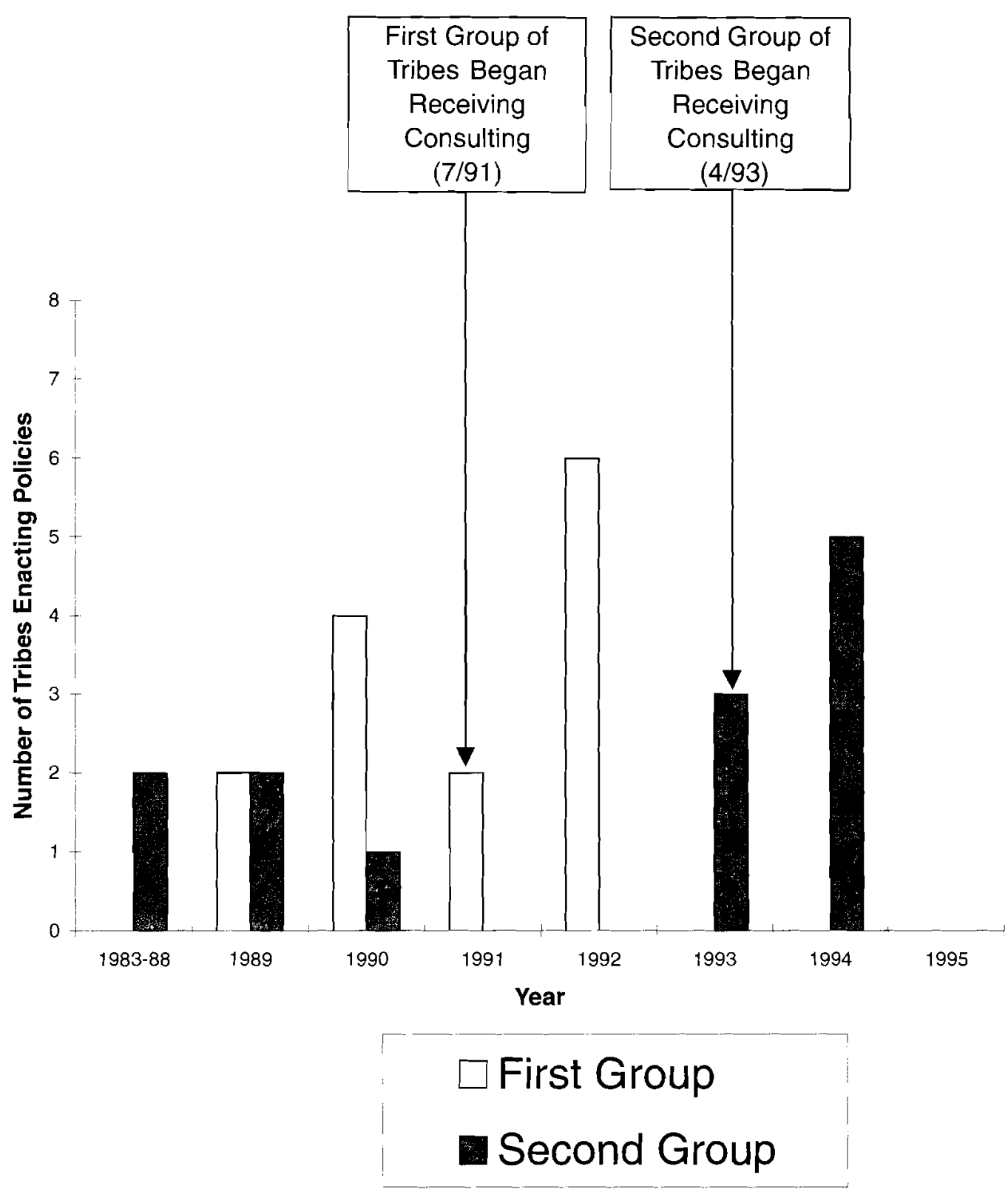

Tribes that received the consulting help first generally maintained their policy gains. While not surprising, this finding highlights one of the advantages of programs aimed at policies: once made, policy changes tend to stay in place. Despite gen- erally positive results, neither group of tribes changed policies on tobacco use in bingo halls and casinos or on reduced youth access to tobacco. In future work with Indian settings, youth access to tobacco, in particular, should be explored. 


\section{Cooperation With and Consequences of Tobacco Use Policies}

Whether the enhanced tobacco use policies will be followed is an important question. We recorded observations of tobacco use in six tribes from each of the two groups of tribes. These records (21) clearly suggest both good compliance with the policies and decreasing acceptance of recreational tobacco use. The observer's field notes reasonably matched information collected in the telephone interviews. The field observations indicated that tobacco was controlled most in tribal government offices, less in public businesses such as restaurants, and least in bingo parlors and gambling casinos.

Another important question is whether policy changes will lead to reduced tobacco use. The project staff sensed greater interest in tobacco cessation than in policy changes among the tribes. The project field directors received many requests to help tribes provide cessation aid to tribal members. Eight of the nine tribes in Oregon sent delegates to a workshop on cessation training. All 39 tribes asked for some kind of assistance, such as support for taking part in the Great American Smokeout, during the course of the project.

\section{Lessons Learned}

The policy project succeeded in the face of some serious roadblocks for the project staff; for example, the importance of tobacco sales income for many tribes, tobacco use by influential tribal members, and the importance of other health and economic issues requiring tribal attention (such as AIDS, health care services, alcoholism, and unemployment). While NPAIHB viewed tobacco control as important for Indian health, it was generally not so for the individual tribes. One aim of the project was to increase awareness of the health hazards of tobacco, cspecially "secondhand" tobacco smoke. Key to success with a tribe was finding a local ally to promote the program. Such allies varied widely across tribes and included a member of the tribe's health committee, a tribal employee, a public health nurse, a community health representative, and a child welfare worker. The child welfare worker enlisted a youth group to help make the successful presentation to the tribal council. Mobilizing Indian youth for tobacco control activities appears to be a useful strategy.
NPAIHB's connections to the tribes were critical to the success of the project. Although NPAIHB passed a resolution supporting the project, and 19 tribes gave letters of support for the grant proposal, the staff still worked hard to gain the cooperation of each tribe through official channels, such as tribal health committees or tribal councils, and through key individuals within each tribe. The 39 tribes that participated in this project varied in size, organization, economic standing, and ways of dealing with health issues.

The three organizations worked well together. Service-oriented NPAIHB and the academically oriented ORI and OSU researchers received recognition beyond the goals of the project. NPAIIIB came to be known in Indian country as expert in tobacco use issues, and its staff has been invited to give talks at numerous national and regional conferences and to give policy workshops and presentations at several Indian settings. The project's workbook has been adapted by Alaskan Native groups and by the California Rural Indian Health Board. ORI and OSU researchers authored papers that were published in refereed journals in their respective ficlds $(12,14,15)$. We have now revised the workbook for generic use in Indian settings, and we are exploring ways to spread the consulting program to Indian settings throughout the United States. The policy approach, which makes use of tribal independence and tribal health committees and councils, could be adapted to address other health problems in Indian country. These might include accident prevention, such as seat belt use policies or prevention of alcohol abuse. Modifying tobacco use policies to limit children's access to tobacco also deserves attention.

\section{REFERENCES}

1. Burhansstipanov L, Dresser C. Documentation of the cancer research noeds of American Indians and Alaska Natives. Native American Monograph No. 1, NIH Publication No. 93-3603. Bethesda, MD: U.S. Department of Health and Human Services, 1993.

2. Sugarman JR, Warren CW, Oge L, Helgerson SD. Using the Behavioral Risk Factor Surveillance System to monitor year 2000 objectives among American Indians. Public Health Rep 1992; 107:449-56. 
3. U.S. Department of Health and Human Services. Reducing the health consequences of smoking: 25 years of progress. A report of the Surgeon General. U.S. Department of Health and Human Services, Public Health Service, Centers for Disease Control, Center for Chronic Disease Prevention and Health Promotion, Office on Smoking and Health. DHHS Publication No. (CDC)89-8411. Washington, DC: U.S. Government Printing Office, 1989.

4. Warner KE. Smoking and health: a 25-year perspective. Am J Public Health 1989;79:141-3.

5. Hall RL, Dexter D. Use of smokeless tobacco among Indian youth in three areas of Washington: Neah Bay, Colville, and Ferndale. Portland, OR: Northwest Portland Area Indian Health Board, 1988.

6. Glantz SA, Parmley WW. Passive smoking and heart disease: epidemiology, physiology, and biochemistry. Circulation 1991:83:1-12.

7. U.S. Department of Health and Human Services. The health consequences of involuntary smoking: a report of the Surgeon General. Public Health Service, Centers for Disease Control, DHHS Publication No. (CDC)87-8398. Washington, DC: U.S. Government Printing Office, 1986.

8. Environmental Protection Agency and National Cancer Institute. Respiratory health effects of passive smoking: lung cancer and other disorders. Smoking and Tobacco Control Monograph 4. NIH Publication No. 93-3605. Washington, DC: U.S. Department of Health and Human Services, Public Health Service, National Institutes of Health, U.S. Environmental Protection Agency, 1993.

9. Lichtenstein E, Glasgow RE. Smoking cessation: what have we learned over the past decade? J Consult Clin Psychol 1992;60:518-27.

10. U.S. Department of Health and Human Services. Strategies to control tobacco use in the Inited States: a blueprint for public health action in the 1990's. Smoking and Tobacco Control Monograph No. 1. NIH Publication No. 92-3316. Rockville, MD: U.S. Department of Health and Human Services, Public Health Service, National Cancer Institute, 1991.
11. Davis RM. Tobacco policy research comes of age. Tob Control 1995; 4:6-9.

12. Glasgow RE, Lichtenstein E, Hall R, Meyers GB, Wilder D, McRae SG. Indoor smoking policies of Indian tribes in the northwestern United States. Tob Control 1993;2:35-7.

13. Glasgow RE, Lichtenstein E, Wilder D, Hall R, Gilbert-McRae S, Liberty B. The Tribal Tobacco Policy Project: working with Northwest Indian tribes on smoking policies. Prev Med 1995;21:434-40.

14. Lichtenstein E, Glasgow R, Lopez K, Hall R, Gilbert-McRae S, Meyers GB. Promoting tobacco control policies in Northwest Indian tribes. Am J Public Health 1995;85:991-4.

15. Hall R, Lichtenstein E, Burhansstipanov L, Davis SM, Hodge F, Schinke $S$, et al. Tobacco use policies and practices in diverse Indian settings. Am Indian Culture Res J 1995;19:165-80.

16. Lichtenstein E, Lopez K, Glasgow RE, GilbertMcRae S, Hall R. Effectiveness of a consultative intervention to promote tobacco control policies in Northwest Indian tribes: integrating experimental evaluation and service delivery. Am J Community Psychol 1996:24:639-55.

17. Glasgow RE, Boles SM, Lichtenstein E, Strycker LA. Tobacco policy rating form: a tool for evaluating worksite and tribal smoking control policies. Tob Control 1996;5:286-91.

18. Glasgow RE, Sorensen G, Corbett K. Worksite smoking control activities: prevalence and related worksite characteristics from the COMMIT study, 1990. Prev Med 1992;21:688-700.

19. Rigotti NA, Pashos CL. No-smoking laws in the United States: an analysis of State and city actions to limit smoking in public places and workplaces. JAMA 1991;266:3162-7.

20. Welty TK, Tanaka ES, Leonard B, Rhoades ER, Hurlburt WB, Fairbanks L. Indian Health Service facilities become smoke-free. JAMA 1987;258:185.

21. Hall RL, Viles CA, Lichtenstein F, Glasgow RE, Lopez KL. Rapid assessment procechures to describe tobacco practices at sites managed by Indian tribes. Tob Control 1995;4:156-61. 


\section{ACKNOWLEDGMENTS}

The work summarized here was supported by National Cancer Institute Grant No. U01CA52230.

We thank G. Bruce Meyers and Bryson Liberty for help with the policy intervention; Gary Brown and Lynn Delorme for conducting the telephone interviews; and Doni Wilder, executive director of NPAIHB, for her advice and support. Co-investigators Russell Glasgow and Robcrtad Hall (OSU) and project coordinator Suzanne McRae were critical to the project's success. Thanks are also due to Shawn Boles and Lisa Strycker for data management and data analyses and Christine Cody for help with manuscript preparation. Lisa Strycker also provided invaluable help in improving the clarity of the paper.

\section{AUTHORS}

\section{Edward Lichtenstein, Ph.D.}

Research Scientist

Oregon Research Institute

1715 Franklin Boulevard

Eugene, OR 97403

Tel: (541) 484-2123

Fax: (541) 484-1108

E-mail: ed@ori.org

\section{Kerri Lopez}

Health Resources Coordinator

Northwest Portland Area Indian Health Board

Suite 335

520 SW Harrison

Portland, OR 97201

Tel: (503) 228-4185

Fax: (503) 228-8182

E-mail: kerri@npaihb.org 


\section{Chapter 6 \\ "It's Your Life-It's Our Future" Stop Smoking Project}

Felicia Schanche Hodge, Larri Fredericks, and Patricia Kipnis

\section{ABSTRACT}

A

team of American Indian researchers used culturally sensitive methods, focus groups, and research data to replicate a National Cancer Institute $(\mathrm{NCl})$ smoking cessation program. This "quit-smoking" program was adapted to the Indian culture in 18 northern California Indian health clinics participating in the project.

The results from the study showed that American Indian adults had extremely high smoking rates, and that 40 percent of American Indian adults were knowledgeable about the harmful effects of smoking and held lenient attitudes toward smoking. Knowledge about the harmful effects of smoking had little effect on smoking behavior. After examination of these and other findings, smoking cessation messages related to three topics: cultural identity, responsibility to family and tribe, and respect for tobacco products were identified as the most culturally sensitive and appropriate to use with this population.

These findings guided the revision of a quit-smoking program that was implemented in four urban and seven rural/reservation clinics in northern California. An evaluation of the program indicated that it had a strong impact on adult Indians' smoking cessation.

\section{INTRODUCTION}

Programs to help people quit smoking have existed for many years in the United States. These programs, however, have had little impact on the American Indian population. Cigarette smoking by adults in the U.S. general population decreased considerably between 1965 and 1985: 52 percent of men and 34 percent of women smoked in 1965, compared with 33 percent of men and 28 percent of women in 1985 (1). Among minority groups and particularly American Indians, smoking rates continue to increase. Smoking rates among American Indians vary by geographic region and by urban or rural residence. In the Southwest, smoking rates are among the lowest at 13 percent (2); in the Northern Plains States, Indian smoking rates are reported to be greater than 50 percent (3). The current smoking rate among the U.S. general population is 21 percent for adults (4).

The 1990 census reports that 1.9 million American Indians and Alaska Natives reside on reservations that are located primarily in 25 States. According to the 1990 census figures, 242,000 American Indians and Alaska Natives live in California $(2,5)$, the second largest concentration of Indians in the country. This population doubled in size between 1970 and 1985 (6) and has increased by 20.3 percent since the 1980 U.S. census. Indians in California are widely 
scattered and highly mobile; they frequently move back and forth between their rural reservations of origin and major urban areas. Eighty-five reservations and rancherias are scattered throughout the State. The two largest reservations, Hoopa and Round Valley, are located in northern California. About 30 percent of California's Indian population reside in rural counties, and the remaining 70 percent are concentrated in five major urban sites: San Francisco, Oakland, Los Angeles, Sacramento, and San Jose (7). Also, many Indians have relocated to California from other parts of the country.

Studies have shown that smoking rates decrease when physicians advise their patients not to smoke ( 8 11). For this reason, smoking cessation advice from physicians and health providers, along with a motivational videotape and followup by a community health representative (CHR), played an important part in "It's Your Life-It's Our Future," a smoking cessation program designed specifically for American Indians in California. This type of project entails understanding the culture and the values of the target population. To adapt existing smoking cessation programs and make them acceptable, available, and accessible, the smoking cessation materials and messages must be understood and accepted by the Indian population. This chapter describes the "It's Your Life-It's Our Future" program and its effect on the smoking status of American Indians in California.

\section{METHODS}

The Center for American Indian Research and Education (CAIRE) is dedicated to improving the health and social welfare of American Indians and Alaska Natives. Governed by an all-Indian advisory board, CAIRE is staffed primarily by Indian professionals. In 1989, the tribal leaders in California were polled to identify priority areas for research. Smoking cessation was identified as the number one area in need of attention.

From this identified need, the "It's Your Life--It's Our Future" smoking cessation project was funded by the NCI in 1990. The program was based in 18 American Indian outpatient clinics (4 urban and 14 rural reservations) in northern California. The project consisted of five phases: (1) working with the
Indian community to identify needs and barriers related to smoking cessation, (2) designing or revising quit-smoking materials, (3) conducting the prevalence survey, (4) implementing the program, and (5) evaluating the program. The principal investigator used these steps to gather support from the Indian communities, to provide the most practical and efficient smoking cessation methods, and to ensure that the project was culturally appropriate and accessible to the community.

\section{Working With the Indian Community}

The urban and rural Indian health clinics that have evolved outside of the Indian Health Service (IHS) system are the centers of community life for American Indians in northern California. These clinics are managed and controlled by all-Indian boards of directors. Because they are the most common facility used by Indians and the central points of contact for this population, these clinics were selected to access the American Indian population recruited into the study.

At the beginning of the project, the principal investigator notified the health boards of all 18 Indian clinics about the project. CAIRE staff met with the clinic health boards to provide information on the project. The most frequently asked questions were the following: how the randomization of sites took place, whether the clinic could be an intervention instead of a control site, what was the availability of the smoking cessation personnel, and how the gathered information would be kept conficlential. participants received information on the importance of confidentiality, participant consent forms regarding the required protection of human subjects, and a description of project tasks and the general rescarch design.

To accomplish the smoking cessation goal, the project used local artwork, worked with clinic staff, and received guidance from local Indian advisors who recommended appropriate artwork, the use of wording, and titles. Indian staff helped identify focus group members and helped administer the questionnaires to participants. Project staff made the findings of the prevalence survey related to smoking and smoking behaviors into a booklet and mailed one copy to all 18 clinics for their use. 


\section{DEVELOPING THE SMOKING CESSATION PROGRAM}

To identify culturally appropriate messages for the smoking cessation intervention project, the authors used targeted focus groups and a questionnaire to gather information on Indian smokers and nonsmokers. This information served as a framework for the development of kcy culturally sensitive messages to increase knowledge and change smoking behaviors in the targeted Indian communities.

Project staff conducted three sets of focus groups (10 to 15 participants in each set) in American Indian communities in urban and rural California to determine beliefs, barriers, and acceptable methods of smoking cessation. Criteria for focus group participation included American Indian ethnicity (smokers, ex-smokers, and nonsmokers), age 18 and older, living in the target communities, and clinic users.

Trained American Indian focus group leaders conducted the 2-hour sessions on key topic categories. Initial contacts for focus group participants were made at the local health clinics. A "snowball" method of contact was adopted: respondents were asked to provide names of other individuals in the community who would fit the study criteria. Smokers and nonsmokers were intervicwed separately in community meeting rooms. Identification as American Indian was self-reported.

Information from the focus group was gathered by the focus group leader in regard to their healthcare beliefs and behaviors, use of formal and informal sources of healthcare, use of traditional healers, traditional use of tobacco, barriers to smoking cessation, and preferred methods of smoking cessation.

Focus groups were used again later to design and pretest the media materials. These materials, a stopsmoking guide and a motivational film, became important parts of the project intervention. Other areas of interest included media preferences, communication styles, presentation of materials, literacy levels, and cultural acceptability.

The final series of eight focus groups (four groups with American Indian adult community members and four groups with adult American Indian health professionals from the Indian clinics) pretested the project's media materials. These focus groups varied in size from 4 to 10 participants. The communication strategies (smoking cessation guide, video, and CHR outreach) were pretested in each of the focus groups. The materials were assessed for cultural acceptability, relevance and message appeal, level of comprehension, accuracy of information, and degree of credibility among the target audience.

After examination of these and other findings, researchers identified smoking cessation messages targeting three topics as the most culturally appropriate to use with this population:

- cultural identity,

- responsibility to family and tribe, and

- respect for tobacco products for ritual use.

\section{CONDUCTING THE PREVALENCE SURVEY}

The principal investigator used information gathered from these focus groups to develop a culturally appropriate survey instrument for use with the project. This survey was administered to 1,369 adult Indians in northern California. Fighteen American Indian clinics participated. Depending on the size of the clinic, 30 to 120 adult Indians age 18 and older were asked to complete a self-administered questionnaire when registering for a medical or dental appointment. The prevalence survey was administered over a 14-month period. Respondents were provided information on the project, asked to read and sign a participation consent form, and told that participation in the project was voluntary and not related to their receiving services at the clinic. Also, they were told that they could refuse to answer any question they did not want to answer. Ninety percent, or 1,369 of those asked, agreed to participate in the project.

The study collected data on demographics, smoking behaviors, readiness to quit smoking, and a number of other characteristics involving knowledge and attitudes about smoking. It also included a social support scale. Participants were classified as current smokers, former smokers, or people who had never smoked. Current smokers were defined as those who reported smoking at the time of the survey. Former smokers reported not smoking at the time of the survey but having smoked at least 100 cigarettes in their lifetime. Respondents who neither smoked at the time of the survey nor had smoked 100 cigarettes were classified as having never smoked. 
We rated the readiness of current smokers to quit smoking on the Prochaska Scale (12) according to three categories: the precontemplative stage (not interested in quitting), the contemplative stage (thinking of quitting within the next 6 months), and the active stage (willing to quit within 30 days). We used a Likert scale to measure individuals' knowledge of the health risks associated with smoking and chewing tobacco and to identify smoking-related behaviors and attitucles. A series of questions addressed the number of close relatives and friends whom respondents felt they could rely on for help or encouragement, and participants were asked about their social support network. The social support network could include others besides close relatives or friends in the context of trying to quit smoking, that is, the clinic community health worker or the clinic physician. A participant receives emotional and social support from a network and is sometimes estranged from relatives/friends or unable to utilize family/friends for this purpose. Other questions determined the number of hassles (stressful experiences) participants encountered during the previous 3 months.

\section{PREVALENCE SURVEY FINDINGS}

The survey results showed that American Indian adults in participating northern California clinics had an extremely high smoking rate of 40 percent $(95 \% \mathrm{Cl}$ $=37.3,42.6$ ). Of the 1,369 adult Indians surveyed, 47 percent of adult males $(95 \% \mathrm{CI}=42.3,51.6)$ and 37 percent of females $(95 \% \mathrm{CI}=33.7,40.3)$ were current smokers. Two of the four urban sites had extremely high smoking rates ( 56 and 40 percent). 'The survey examined the relationship between smoking and selected demographics. Divorced (45 percent, $95 \% \mathrm{Cl}$ $=38.9,51.0$ ) and single ( 44 percent, $95 \% \mathrm{Cl}=38.9$, 49.1) participants had a higher smoking rate than did those who were married ( 36 percent, $95 \% \mathrm{CI}=32.0$, 39.9 ) or widowed ( 23 percent, 95\% CI $=13.0,33.0$ ). The unemployed and welfare recipients had a 56 percent smoking rate $(95 \% \mathrm{Cl}=48.8,63.2)$, much higher than the 40 percent rate of the entire sample. Individuals with less than a high school education were more likely to be current smokers than were those who had completed high school.

Usban participants (men in particular) were more likely than rural participants to be current smokers.
Forty-three percent of rural Indian men were currently smoking compared to 57 percent of urban Indian males. Urban Indians were more likely than Indians living in rural areas to be unemployed or on welfare. Urban Indians also were more likely to experience stressful events such as being victims of theft or living in unsafe neighborhoods. Urban Indian smokers were more motivated to quit smoking than were Indians living in rural areas. Twentynine percent of American Indian smokers in urban areas were ready to quit smoking within a month, compared to 20 percent of Indian smokers in rural areas

Key prevalence survey findings are listed below:

- The highest percentage of adult smokers was among members of the Sioux tribe (62 percent, $95 \% \mathrm{CI}=50,74$ ). The following smoking rates were found among native California tribes: Maidu ( 46 percent, 95\% Cl = 34, 57); Pit River (39) percent, $95 \% \mathrm{CI}=25,54)$; Pomo ( 38 percent, $95 \% \mathrm{CI}=30,47$ ); and Hupa (37 percent, $95 \% \mathrm{CI}$ $=26,48$ ).

- The number of cigaretues smoked was statistically correlated with having alcohol-related problems. For example, heavy smokers were more likely to be heavy drinkers.

- Male smokers reported smoking, on the average, more than women did, smoking an average of 18 cigarettes per day. Indian men reported having started smoking at a younger age than did women. Indian men also were more likely to have alcohol problems than were Indian women.

- Indian women were more likely to report smoking for weight control than were Indian men. Also, a higher percentage of former smokers than current smokers or nonsmokers suffered from being overweight, particularly women

- Fourteen percent of Indian males and 2 percent of Indian females used smokeless tobacco, compared with 5.2 percent of males and .01 percent of females in the general population.

- Evidence indicates that alcohol and tobacco use begins at a very early age. Northern California Indians report that, on the average, they began to smoke cigarettes at age 15 . Some began smoking as young as age 10 . 
- Smokers were as knowledgeable about the harmful effects of smoking as nonsmokers were. However, their attitudes differed significantly from former smokers and nonsmokers.

\section{EVALUATING THE PROGRAM}

The "It's Your Life--It's Our Future" smoking cessation program consisted of three important parts: (1) the identification of the clinic client as a smoker, (2) the delivery of a stop-smoking message from the physician to the smoker, and (3) a followup visit from the CHR who provided educational material and support to help the smoker stop smoking and remain a nonsmoker. The researchers instructed the clinics on the NCI approach to maintaining office records and followup techniques. Clinic staff were responsible for placing a smoking sticker on each smoker's clinic record, inserting a clinic visit reminder sheet in each file, and instituting followup recordkeeping. The CHR showed a motivational film (It's Your Life) to the smoker and provided a selfhelp guide, both of which were developed specifically for American Indian smokers. For those desiring to quit smoking, the clinic physician provided smoking cessation assistance in the form of nicotine gum, nicotine transdermal patches, and counseling. The self-help guide highlighted information on various techniques to quit smoking, such as physical activities and removing ashtrays from the home. The CHR provided two additional followup visits to the smokers, generally in their homes, or if requested, at the clinic.

Fourteen Indian health clinics in northern California participated in a randomized community trial to evaluate the program. The remaining four clinics did not participate because they were small rural clinics with extremely low numbers of patients. Seven clinics were randomly selected to participate in the "It's Your Life-It's Our Future" smoking cessation program. The other seven clinic sites provided regular medical care to their patients.

Smokers who agreed to participate in the study completed a baseline questionnaire on their demographic characteristics, smoking habits and history, willingness to quit, and their knowledge, attitudes, and practices related to smoking. A household was considered part of the study when one member enrolled in the program. A total of 1,369 smokers participated in the prevalence study. Most smokers ( 86 percent) were age 18 to 50,57 percent were women, and 29 percent were unemployed.

The researchers contacted and surveyed study participants at control and intervention clinics twice after the baseline period: (1) 6 to 8 months after the initial contact, and (2) 18 months after the initial contact. Seventy-five percent of baseline participants completed a questionnaire at the 6-month followup, and 62 percent completed a questionnaire at the 18 month followup. For statistical purposes, individuals who dropped out of the program before its completion were assumed to be smokers.

\section{QUIT-SMOKING PROGRAM FINDINGS}

The program was evaluated both at the 6-month followup and the 18-month followup.

\section{6-Month Followup}

Smokers were considered to have quit if they had not smoked for at least a month prior to the 6-month followup. The percent of smokers who quit smoking at the clinics, following the "It's Your Life-It's Our Future" program, was 6.8 percent $(95 \% \mathrm{CI}=5.0$, 8.9), compared to 3.4 percent $(95 \% \mathrm{Cl}=2.1,4.9)$ for clinics providing standard care. The difference between the two groups was significant $(p=.016)$. When controlling for other factors that were related to smoking cessation, such as motivation to quit, number of cigarettes smoked, and relatives who smoke, the difference was still significant.

Smokers were more likely to quit if they were younger, wcre female, did not have a smoker in the house, smoked less than 12 cigarettes a day, did not smoke within 30 minutes of waking up, and were highly motivated to quit.

For individuals who were not interested in quitting at baseline, the program effect was 3.8 percent (difference between participating clinics and clinics providing standard care, $95 \% \mathrm{CI}=2.3,5.4$ and $\mathrm{p}=$ .062). For individuals who were thinking about quitting within the next 6 months at baseline, the program effect was 4.5 percent $(95 \% \mathrm{CI}=2.6,6.9$ and $\mathrm{p}=125$ ). For individuals who were ready to quit at baseline, the intervention effect was 7.2 percent $(95 \% \mathrm{CI}=4.5,10.2$ and $\mathrm{p}=.11)$. After 6 months, the program had a greater effect among those who were interested in quitting at baseline than among those 
who were not. The program had a greater effect on light smokers than on heavier smokers.

\section{8-Month Followup}

The percent of smokers who had not smoked for at least a year before the 18-month followup in clinics following the "It's Your Life-It's Our Future" program was 5.7 percent $(95 \% \mathrm{CI}=4.1,7.7)$, compared to 3.1 percent $(95 \% \mathrm{CI}=1.9,4.6)$ for clinics providing standard care $(p=.07)$. The effect of the program. according to motivation to quit at baseline (or first questionnaire), was similar to that found in the 6-month followup questionnaire. The program was more effective for individuals who were more motivated to quit at the beginning of the program and had a greater effect on lighter than on heavier smokers.

Thirty percent of smokers at clinics promoting the program had attempted to quit during the study, compared to 20 percent at clinics providing standard care. Smokers who did not quit at the end of the program were more interested in quitting smoking in the next 6 months in clinics participating in the program versus smokers attending clinics providing standard care.

The percentage of families with members who attempted to quit smoking during the study in clinics providing standard care was 6.8 percent, compared to 11.1 percent at clinics participating in the program $(\mathrm{p}=.046)$. The percentage of families with members who quit smoking during the study in clinics providing standard care was 2.9 percent, compared to 5.4 percent at clinics participating in the program $(p=.19)$.

\section{DISCUSSION}

This study is significant because it documents that smoking is a very serious problem in American Indian communities in California. The results of the prevalence survey showed an extremely high smoking rate of 40 percent. Urban clinic sites had a higher smoking rate than rural clinic sites. Indian men had the highest smoking rates; Indian women's smoking rates, although lower than Indian men in California, were higher than the smoking rate of California's general population-20 percent. The high incidence of smoking and its effects on the health of American Indians has been well documented by other studies (13-15). The Indian Health
Service has reported that two out of every five Indian deaths in the United States are due to smoking (3). In California, Indian women die of lung cancer at four times the rate of non-Indian women (16). Other health problems associated with smoking cigarettes, such as heart disease, stroke, and other cancer deaths, are also increasing in Indian communities.

The "It's Your Life-It's Our Future" project provided valuable statistics and program information for planning and instituting smoking cessation programs that were effective and culturally appropriate for American Indians. At the beginning stages of the program, community and clinic professionals were invited to offer input into the project planning through focus groups. Direction provided by focus groups yielded valuable insight into how to access the Indian population and target smoking cessation effectively. Clinic providers were instrumental in getting the "quit" message to their Indian clients and referring them into the program. The Indian CHRs were also valuable in providing followup and encouragement to participants following the "quitsmoking" program. The implemented program was successful and culturally sensitive to the American Indian population. The results of the project show that 30 percent of the smokers at the clinics promoting the program attempted to quit smoking during the study.

\section{CONCLUSION}

The "It's Your Life-It's Our Future" smoking cessation project has succeeded in two ways. First, it provides important information for Indian leaders and health planners because it documents that smoking is a very serious problem in American Indian communities; second, it serves as a model for implementing a culturally sensitive smoking cessation program. Studies have shown that American Indians have some of the highest smoking rates in the Nation. This is particularly true in northern and western states. In California, we have documented some of the highest rates of smoking: for example, 40 percent of adult Indians in northern California smoke cigarettes, compared to 25.5 percent (17) of adult smokers in the U.S. white population and 20 percent of adult smokers in California's general population. 
Attention must be given to the creation of smoking cessation programs designed specifically for American Indians. Tailoring programs to the American Indian population requires an understanding of the culture and an ability to work from within so that education intervention programs are acceptable and accessible to this population. The "It's Your Life-It's Our Future" smoking cessation project is a model that is both effective and culturally appropriate for American Indians.

\section{REFERENCES}

1. Batinder R. Tobacco, the jurist's viewpoint. In: Slama K, editor. Tobacco and health. New York: Plenum Press, 1995:77-90.

2. The National Cancer Institute. Addendum for the Native American Indian Women's Cancer Initiative. Public Health Service, National Institutes of Health, 1992.

3. Oregon Research Institute. Northwest Portland Area Indian Health. Tribal tobacco policy workbook. Portland: Oregon State University, 1996.

4. Bennett T. Unpublished findings from A Study of California Indian Health Needs. Report Mandated by Congress, Public Law 100-713. San Francisco: University of California Press, 1992.

5. U.S. Bureau of the Census. Census of the population: general population characteristics. Washington, DC: U.S. Department of Commerce, 1992.

6. Hodge F. Analysis of American Indian health care needs in northern California. Report to the Sierra Foundation. Berkeley: University of California, 1989.

7. Health Status and Health Care Needs of American Indians in California. Report to Congress on Indian Health Service. Public Law 100-713, Section 709. 1991.

8. Cummings S, Coates $T$, Richard R, Hansen B, Zahnd E, Vander Martin MS, et al. Training physicians in smoking cessation: a randomized trial of the "Quit for Life" program. Ann Intern Med 1989;110:640-7.

9. Russell M, Wilson C, Taylor C, Baker CD. Fffects of general practitioners' advice against smoking. Br Med J 1979;2:231-5.
10. Wilson D, Wood G, Johnson N, Sicurella J. Randomized clinical trial of supportive followup for cigarette smokers in a family practice. Can Med Assoc J 1982;126:127-9.

11. Jamrozik K, Vessey M, Fowler G, Wald N, Parker $G$, Van Vunakis H. Controlled trial of three different antismoking interventions in general practice. Br Med J (Clin Res Ed) 1984;288:14991503.

12. Prochaska J, Velicer W, DiClemente C, Fara J. Measuring processes of change: applications to cessation of smoking. J Consult Clinical Psychol 1988;56:520-8.

13. Klatsky AL, Siegelaub AB, Landy C, Friedman GD. Racial patterns of alcoholic beverage use. Alcohol Clin Exp Res 1983;7:372-7.

14. U.S. Department of Health and Human Services. The health consequences of smoking: cancer, a report of the Surgeon (ieneral. DHHS Publication No. 88-8406. Washington, DC: Superintendent of Documents, U.S. Government Printing Office, 1982.

15. Bobo JK, Gilchrist LD. Urging the alcohol client to quit smoking cigarettes. Addict Behav 1983; 8:297-305.

16. Northern Arizona University, Institute for Human Development, Arizona University Affiliated Program. Facts and figures on American Indians and Alaska Natives. American Indian Rehabilitation Newsletter: Newsletter for American Indian Rehabilitation Research and Training Center 1994;10:1.

17. U.S. Department of Health and Human Services, Public Health Service, Centers for Disease Control and Prevention (CDC). Frequent smoking among adults-United States, 1991. Mor Mortal Wkly Rep 1993;42:230-3.

\section{ACKNOWLEDGMENTS}

Funding for this study, Smoking Cessation for American Indians, was provided by Grant No. U01CA52279 from the National Cancer Institute. 


\section{AUTHORS}

Felicia Schanche Hodge, Dr.P.H.

Director

Center for American Indian Research and Education 1918 University Avenue, Suite 2A

Berkeley, CA 94704

Tel: (510) 843-8661

Fax: (510) 843-8611

E-mail: feliciash@aol.com

\section{Larri Fredericks, Ph.D.}

Research Associate

Center for American Indian Research and Education 1918 Inniversity Avenue, Suite 2A

Berkeley, CA 94704

Tel: (510) 843-8661

Fax: (510) 843-8611

E-mail: larrif1@aol.com

\section{Patricia Kipnis, Ph.D.}

Statistician

Kaiser Permanente Medical Center 280 West MacArthur Boulevard

Oakland, CA 94612 


\title{
Chapter 7 \\ Pathways to Health: A Cancer Prevention Project for Native American Schoolchildren and Their Families
}

\author{
Sally M. Davis, Leslie Cunningham-Sabo, and Lori C. Lambert
}

\section{ABSTRACT}

$\mathrm{T}$

he Pathways to Health project is a school-based program of cancer prevention and health promotion activities for fifth- and seventh-grade American Indian students. The curriculum was implemented in 12 elementary and middle schools located in rural communities in New Mexico. The 5-year program was designed to be culturally relevant for rural American Indian children in the Southwest by incorporating American Indian traditions and values into lessons and activities. The curriculum objectives related to promoting a diet low in fat and high in fiber, fruits, and vegetables as well as avoiding nonceremonial cigarette smoking and use of smokeless tobacco products. The project activities included parents and grandparents. Participating schools were randomly assigned to one of three conditions: cancer prevention curriculum, cancer prevention curriculum augmented with a family intervention, or control (delayed intervention). Qualitative data collected from focus groups and individual interviews indicated wide acceptance and use of the program. Changes in self-reported use of tobacco and in attitudes toward tobacco indicate positive effects of the intervention. Additional analyses of data regarding nutrition and other aspects of the curriculum will be reported in the future.

\section{INTRODUCTION}

Many things people do in their day-to-day life influence how long they will live. For example, about 30 percent of all cancer deaths are caused by tobacco use (1). About 50 percent of cancer incidence is related to the foods we eat $(2,3)$. Both the National Cancer Institute (NCI) and the American Cancer Society (ACS) encourage us to eat fewcr fatty foods, to eat more foods with fiber, and not to use tobacco products (4.5). Another important step in having a healthy life and preventing cancer is keeping a healthy weight. All of these behaviors are related to the choices we make in our everyday life--whether or not we smoke or chew tobacco, what foods we choose to eat and how we prepare them, and how well we control our weight.

In the past, strong cultural traditions and living in rural areas away from the influences of urban life protected many Southwestern American Indian people from some risk factors associated with cancer. However, this protection is disappearing, and many Native people are adopting Western lifestyles that often hring unhealthy habits (6). These lifestyle changes are likely to lead to an increase in cancer risk factors and disease among American Indians in the Southwest.

Death from lung cancer among Native groups living in Arizona and New Mexico is less likely than 
among (other U.S. tribes (7). Southwestern tribes typically have a smaller proportion of people who are heavy smokers, which may account for these differences. However, prevalence of diseases related to tobacco use may increase as more Southwestern Indians adopt Western ways (8). Recent research shows that tobacco use among Indian youths in the Southwest is increasing (9). In recent years, more and more teenagers and young adults have started using smokeless tobacco (10-13). Although mainly boys and young men use spit tobacco in most cultures, young American Indian women are becoming more likely to use smokeless tobacco products (14-17).

The number of cancers related to diet among American Indians remains generally lower than that in the general U.S. population (18). Iowever, the risk of these cancers as well as diabetes and cardiovascular disease may be increasing as American Indians select more foods higher in fat and lower in fiber and obesity rates continue to increase (19). Recent studies of American Indian children in Arizona and New Mexico document a significant rate of obesity (20-22). Like children elsewhere in the United States (23), American Indian children's food intake is high in fat, with approximatcly 35 percent of daily calories coming from fat (22).

Schools can help young people develop healthful lifestyle behaviors (24). Cultural beliefs and practices can have a very strong influence on decisions made in daily living, including those choices related to personal health (25). For American Indians, desired health habits must be modeled and reinforced within their own cultural context to succeed in bringing about favorable lifestyle changes (26).

In response to growing concern among conmunity members, familics, school employees, healthcare providers, and health educators, a group of health promotion and disease prevention workers from Native American communitics in northwestern New Mexico and the University of New Mexico developed, implemented, and evaluated Pathways to Health, a cancer prevention curriculum specifically for American Indian children and their families. The specific aims of this project were threefold:

1. Develop, implement, and evaluate a multidisciplinary cancer prevention program for use with fifth- and seventh-grade children and their families for several American Indian tribes in the Southwest. Specific tasks are described below.
- Design a program to pronote the avoidance of smoking and the use of smokeless tobacco and to promote a low-fat, high-fiber diet.

- Design, implement, and evaluate the effectiveness of a health promotion curriculum in terms of changed health behaviors and attitudes and increased knowledge regarding tobacco use and diet.

- Implement intergenerational activities designed to reinforce the curriculum in culturally relevant ways.

- Develop and evaluate culturally appropriate audiovisual and written educational materials on cancer, tobacco, and healthful diet.

- Compare the effectiveness of the curriculum as taught to students in the fifth grade to the effectiveness of the curriculum taught to students in the seventh grade.

- Compare the effectiveness of the curriculum as taught only at school to the effectiveness of the curriculum supplemented by a family intervention.

2. Design, implement, and evaluate with qualitative measures a training program that effectively incorporates school-based staff to ensure programmatic continuation.

3. Provide demographic, normative, and physical measurement data on the characteristics of this population with respect to risk factors for future cancer in adulthood.

This chapter describes the development and implementation of the Pathways to Health project. Preliminary pretest and posttest data from the survey on tobacco use and attitudes are presented. Additional analysis of the effectiveness of the Pathways to Health project will be presented in the future.

\section{METHODS}

\section{Population}

A total of 1,589 students predominantly from two tribes in the Southwest were pretested in the 3 school years between fall 1992 and spring 1995. Of these, 825 ( 51.9 percent) were boys and 764 ( 48.1 percent) were girls, 815 (51.2 percent) were in the fifth grade and 774 (48.7 percent) were in the seventh grade, 
and 1,494 (94 percent) were American Indian. The 97 students that reported other ethnic backgrounds were predominantly non-Hispanic white and Hispanic white. All schools were located in rural areas and were located on or adjacent to Indian reservations serving predominantly Indian children.

Students who were in the fifth grade in the 19921993 school year (year 1) also participated in the program as seventh graders during the 1994-1995 school year (year 3). All other students participated only when they were in either the fifth or seventh grade. Tobacco results presented in this chapter include data only from years 1 and 2 of the project; therefore, the results do not include data from students who received the curriculum twice. In future analyses, students who received the curriculum both in the fifth and seventh grades will comprise an additional comparison group to cvaluate the effectiveness of receiving the curriculum twice versus once.

\section{Research Design}

The Pathways to Health project used a pretest and posttest design, with schools randomly assigned to one of the following groups: curriculum only; curriculum plus family; or control (delayed intervention). The curriculum-only schools received the cancer prevention curriculum taught by classroom teachers. The curriculum-plus-family schools received the same cancer prevention curriculum along with a family intervention component. The family component was developed and piloted in year 1 and added in years 2 and 3 of the study. The four control (delayed intervention) schools received an alternative health education curriculum initially and the Pathways to Health curriculum in the final year of the project (year 3). The control schools received an alternative curriculum at the request of the communities because it was unacceptable for schools to participate in pretesting and posttesting without receiving a benefit in return. The number of schools and students participating in each intervention group for the 3 years of the project are presented in Table 1 . Each year, all participating students (including those attending control schools) completed a series of surveys before and after the program was implemented to assess their knowledge, attitudes, and reported behaviors related to the curriculum.

\section{Process}

Beginning in the fall of 1990 , the project received funding for 5 years (Table 2). The process of developing, implementing, and evaluating Pathways to Health followed a participatory research model (27). This model recognizes the benefits of partnership between those with the scientific and technical knowledge and those with the equally valuable personal and cultural knowledge of the problems the research is seeking to resolve.

The research team spent the first year developing the classroom curriculum and identifying participating schools. The primary objectives of the first year of the project were to establish and renew relationships with community and school personnel; to identify and modify, where appropriate, existing programs, materials, and instruments; to network with Indian Health Service and tribal public health staff; to recruit schools to participate; to test the feasibility of various components of the program; to train staff; to determine the environmental barriers to the project; and to assess the availability of low-fat and high-fiber foods in the participating communities.

The focus of the second year was on pilot-testing and revising the curriculum. Community membersincluding teachers, parents, grandparents, students, school administrators, and project staff originally from the participating communities-contributed to developing the Pathways to Health curriculum and survey instruments. The qualitative and quantitative methods used included focus groups, direct observation, informal interviews, store surveys, and the use of process evaluation data from previous studies in the same schools and communities. In the last 3 years of the project, Pathways to Health was implemented in 12 American Indian schools in rural New Mexico.

\section{Curriculum}

Regular classroom teachers learned the curriculum at a 2-day teacher training program. The Pathways to Health team visited each school on a regular basis to provide technical assistance with the curriculum activities. The teachers received an easy-to-use teacher guide, student workbooks, and other materials needed to teach the curriculum.

The curriculum was developed specifically for fifthand seventh-grade students because children at these ages are already making many decisions about their 


\section{TABLE 1: Number of Schools and Students Participating in the Pathways to Health Project ${ }^{\mathrm{a}}$}

From 1992 to 1995

\begin{tabular}{lccc} 
Year 1 & Curriculum only & Curriculum plus family & Control \\
\hline Fifth grade & 5 schools & b & 1 school \\
& 213 students & b & 23 students \\
Seventh grade & 5 schools & $\mathrm{b}$ & 2 schools \\
& 184 students & $\mathrm{b}$ & 154 students
\end{tabular}

\begin{tabular}{|c|c|c|c|}
\hline Year 2 & Curriculum only & Curriculum plus family & Control \\
\hline \multirow[t]{2}{*}{ Fifth grade } & 4 schools & 2 schools & 1 school \\
\hline & 193 students & 69 students & 30 students \\
\hline \multirow[t]{2}{*}{ Seventh grade } & 3 schools & 3 schools & 2 schools \\
\hline & 122 students & 69 students & 132 students \\
\hline
\end{tabular}

Year 3

Fifth grade

Seventh grade
Curriculum only

5 schools

178 students

4 schools

232 students
Curriculum plus family

Control
2 schools

65 students

4 schools

136 students

"A total of 1,782 students were enrolled in participating schools. Due to absences during pretesting and posttesting, not all students who participated in the curriculum were tested.

${ }^{b}$ In year 1 of the project, the family component was being developed and was not added until year 2.

- Control scbools received an alternate curriculum in years 1 and 2. In year 3, the control schools received the Pathways to Health curriculum; therefore, year 3 bad no control group.

health and encounter peer pressure and other social influences. During the 2 years that the curriculum was being developed, pilot-tested, and revised, the project team held separate focus groups with American Indian teachers, parents, and school administrators from participating schools. The project sought their advice on key cultural concepts and appropriate methods for use in their schools and communities. The following cultural beliefs and values were recommended and used to design the curriculum: learning through observation and practice (28); learning from storytelling (28); learning with examples (metaphorically) (29,30); holistic learning (31); learning by trial and error (32); learning cooperatively (33); and learn- 


\section{TABLE 2: Timeline of Pathways to Health Project Implemented in Native American Communities in Rural New Mexico}

Fall 1990 to Spring 1991

Fall 1991 to Spring 1992

Fall 1992 to Spring 1993-

Year 1

Fall 1993 to Spring 1994-

Year 2

Fall 1994 to Spring 1995-

Year 3
Develop curriculum

Randomly assign schools

Pilot-test and revise curriculum

Begin curriculum-only vs. control interventions

Develop family curriculum

Continue curriculum-only vs. control

interventions and add curriculum-plus-family intervention

Control schools receive curriculum-only

intervention; others schools continue as previously assigned ing through reflection (34). The Patbways to Health curriculum incorporates these cultural concepts into 16 lessons that use activities and storytelling. The familiar trickster character of Coyote is used to teach children how to avoid being "tricked" by their peers and others who try to get them to do unhealthy and risky things. The lessons feature a health promotion curriculum to prevent tobacco use and encourage children and their families to eat foods with less fat and more fiber. Pathways to Health includes lessons on peer pressure, classroom visits from grandparents, storytelling, family activities, and training for teachers and educational assistants.

Following a model developed and used with the Checkerboard Cardiovascular Curriculum (35) and the Southwest Cardiovascular Curriculum (36)two earlier projects developed for American Indian children living in the same areas--the lessons include activities that involve grandparents. Elders from the local communities are included as teachers in the curriculum to teach the children about traditional American Indian ways that recognize the importance of staying healthy. These presentations by the elders give valuable information to the children about their own culture and the ways of their ancestors (37). Students also interview their parents and grandparents about "the old ways," when they grew much of the food they ate, were more physically active, and saved tobacco for sacred uses.

At the base of Pathways to Health is social learning theory (SLT) (38), which promotes selfefficacy and emphasizes the interactions between an individual's attributes and his or her environment and health-related behaviors. For example, the project teaches children not only the importance of selecting and preparing a diet low in fat and high in fiber and avoiding tobacco, but also promotes an environment that supports children in making positive behavior changes. The lessons showcase positive role models and give children opportunities to practice saying no to tobacco. The project creates peer support for selecting lower fat foods and expands children's skills, experience, and confidence in reading food labels and selecting healthful foods.

The curriculum development team developed written materials and a videotape, Life in Balance, as part of the curriculum and the family activities. In the videotape, six American Indian adults serve as role models and describe their personal journeys to reach a balance in their lives through healthful choices. The curriculum development team took extreme care in creating curriculum materials, such as the teacher guide, student workbook, and family activities, to make them culturally appropriate. 


\section{Family Component}

The Pathways to Health family-based activities were developed during the 1992-1993 school year (Table 2). Project staff held focus groups with parents to find out what they thought would work; subsequently, lessons and activities were developed based on this input. The team designed the family materials using some of the techniques described by Doak and co-workers (39). In the fall of 1993, the 5week family intervention began with a kickoff party at the participating schools, followed by 3 weeks of take-home activity packets for the students to complete with one or more family members. The final activity, a wrap-up party-again held at schoolinvited students and family members to celebrate the program. Students received the classroom curriculum concurrently with the family intervention.

\section{Educators' Training}

School staff members were included in all aspects of the Pathways to Health program to make sure that it was accepted in the school and that it was likely to be continued after the funding ended. Teachers and teacher aides attended a 2-day educators' training, held at the University of New Mexico, before teaching the curriculum. They learned why the program was important as well as the content of and methods for teaching the curriculum. The research team demonstrated sample lessons, and elders from participating communities presented stories and information about healthful traditional foods to the teachers. The teachers and teacher aides could receive university credit for completing the training and teaching the curriculum.

\section{Instruments}

To collect descriptive information and evaluate the health promotion curriculum, Pathways to Health staff developed, pilot-tested, and further refined evaluation instruments. These measures included questions from each of three domains: (1) attitudes about health, food, tobacco, and social influences; (2) knowledge of cancer, tobacco, nutrition, and resisting social pressures; and (3) behavior regarding tobacco habits and frequency of eating selected food items. Development and content of instruments are described in detail elsewhere (40-42). Only data from questions pertaining to tobacco use and attitudes are included in this chapter.
Project staff administered all instruments in the classroom before and after the curriculum took place. All students present on the day of testing completed the instruments. Project staff read questions aloud twice to fifth-grade students and once to seventh-grade students to help poor readers understand the questions. Project staff received training to conduct measurements using standard procedures, and classroom teachers did not participate in pretesting or posttesting students.

\section{Process Evaluation}

The research team collected descriptive data from classroom teachers regarding use of and attitudes about the curriculum and teacher training. Teachers completed an evaluation of each lesson and an interview with project staff at the end of each school year. During the final year of the project, an outside interviewer met with each teacher to determine the level at which he or she was using the curriculum. Gene Hall, developer of the levels of use classification system (43), trained and certified the interviewer, who was not a member of the project staff. The research team measured participation of families in take-home activities and attendance at the kickoff and wrap-up parties to assess the acceptance of the family intervention.

\section{Data Analysis}

This chapter presents data on tobacco use and intention to use. In this preliminary analysis, we compared students who received an intervention (curriculum only or curriculum plus family) with students in the control group (delayed intervention).

To assess differences between the pretest and posttest, we assigned students' pretest answers to categories determined by their posttest answers. For example, students who reported at pretest "don't use cigarettes" were matched with their posttest responses (don't use, sometimes use, regular use) to create the new combined categories: don't use/don't use; don't use/sometimes use; and don't use/regular use. The research team made comparisons of pretest and posllest categories between control and intervention students using chi-square tests of significance. For all analyses, individuals were the unit of analysis and the alpha level was set at 0.05 . 
The research team is analyzing additional data from the Pathways to Health study, which will be presented in the future. Statistical analyses of the comparison of student pretest and posttest results between curriculum-only schools and curriculumplus-family schools are in progress.

\section{RESULTS}

\section{Process Evaluation}

\section{Teacber Feedback}

In general, evaluations of lessons were positive, although some activities were more popular with a particular grade level (for example, fifth graders were more involved in performing the wellness rap than seventh graders). Tasting different foods worked well in most classes, as did all of the hands-on activities. Most teachers found the presentations by the elders to be an extremely positive experience for both the students and the elders who participated. The major concern was wishing they had more time to spend on the curriculum during the school day.

Comments from teachers during interviews with project staff included the following: The students enjoyed the hands-on nature of the curriculum; they enjoyed tasting new foods; and teachers found the use of the sponge lungs to demonstrate the effect of tobacco smoke on lungs and the graphic pictures of diseased oral tissue from chewing tobacco to be useful teaching strategies. The teachers appreciated the cultural aspects of the curriculum, and comments on the grandparent/elder activities were always positive.

Results from teacher interviews with the person certified in the levels of use classification system (who was not a member of the project staff) (43) showed the curriculum was well utilized. Of the 17 interviewed teachers, 8 reported "routine" use, defined as stabilized use with few if any changes made in ongoing teaching of the curriculum. Six other teachers had moved to the later stage of "refined" use, where the teacher varies the use to increase the impact on students. Two teachers were at the "mechanical" level, in which the effort is on the short-term, day-to-day use of the curriculum. Only one teacher was classified as a "past" user, who no longer taught the curriculum.

\section{Educators' Training}

The teacher and teacher aide responses to the training program were very positive. Table 3 presents teachers' evaluations of the training, representing the responses of 57 participants at 3 years of trainings. Many of their written comments praised the program: "The curriculum would be marvelous for all grade levels"; "All children need this curriculum!"; "Thanks to everyone responsible for this valuable training that will help us and our community!"; "Very good hands-on training"; and "One of the best I've attended as far as introduction to a new curriculum, plus it provides the most materials."

\section{Family Component}

More families participated in the kickoff party, wrapup party, and completion of take-home assignments than most teachers anticipated. Kickoff party attendance of families ranged from 27 percent in one school to 86 percent in another school. Participation rates were similar for the wrap-up party event. Given the extreme distance from home to school typical in some communities, and that the school with the lowest participation rate is a boarding school, this range of results was not surprising. Often, more than one family member attended these events with the student. Participants rated the various "learning stations" at the kickoff party very highly, with 87 percent giving the event an overall rating of "great." The most frequent responses to "Do you think you are going to do anything different to stay healthy?" were eat less fat, eat healthier foods, and exercise more.

Take-home assignments included students' setting healthy food choice and physical activity goals with their families and reading the "Nutrition Facts" label on packaged foods at the grocery store to identify foods lower in fat and higher in dietary fiber. Participation in take-home assignments ranged from 27 percent in one seventh-grade class to 69 percent in one fifth-grade class. A higher percentage of students completed these worksheets if the teacher was actively involved and reminded them to do this homework. The incentives provided by the project (such as sports water bottles and colorful pens and pencils) were well received, especially by the fifth graders and their families. Fifth graders also were more likely than seventh graders to turn in completed worksheets with evi- 
TABLE 3: Evaluation of Educators' Training by 57 Participants in the Pathways to Health Project, 1992 to 1995

Yes No Somewhat

1. Overall, was the workshop beneficial to

your understanding of the Patbways to

Health curriculum?

56

0

1

2. Prior to the workshop, was the information

about location, time, agenda, parking, etc,

presented in an understandable format and

timely manner?

43

5

8

3. Were the instructors well prepared for the presentations and knowledgeable about

their topics?

55

0

2

dence of family participation. The older students responded that they had too much homework and had little time to complete the Pathways assignments (which held a lower priority because they did not affect their grade). Often, they did not share the project worksheets with their parents.

\section{Changes in Self-Reported Tobacco Use and Intention To Use}

\section{Smokeless Tobacco}

No significant differences were found hetween fifthgrade intervention and control students for questions on smokeless tobacco use. Approximately 92 percent of fifth-grade intervention and control students reported they were not current users of smokeless tobacco at both pretest and posttest. Nearly 65 percent of both groups indicated they would not use tobacco even if their best friend offered it to them. At the posttest, 68 percent of fifth graders were sure they would never use smokeless tobacco, 7 percent thought they would use smokeless tobacco someday, and 25 percent were unsure. This distribution was not significantly different from responses at the pretest. Rates of reported use showed no trends from pretest to posttest, with 2 percent of students ( 7 divided by 395 ) reporting regular use of smokeless tobacco at the pretest and 3 percent (10 divided by 395 ) at the posttest. Similarly, 3 percent ( 15 divided by 430 ) of students reported use of smokeless tobacco within the last 24 hours at the pretest, compared with 3 percent ( 14 divided by 430) at the posttest.

Self-reports of smokeless tobacco use and intention to use among the seventh-grade students (Tables 4 and 5) show differences between the intervention and control groups. Among those who were not current users at pretest, nearly 92 percent of intervention students remained nonusers at posttest, compared with 82 percent in the control group ("don't use/don't use," Table 4). Approximately 5 percent of the intervention nonusers became users by posttest, compared with more than 9 percent in the control group ("don't use/sometimes or regular use," Table 4).

In addition, a significant difference existed between the seventh-grade intervention and control students in expected use if a best friend offered smokeless tobacco (Table 5). Fewer of the control students who said at pretest that they would not use 
TABLE 4: Self-Reported Smokeless Tobacco Use Among Seventh Graders Who Participated in the Pathways to Health Project, 1992 to 1995

\begin{tabular}{|c|c|c|c|c|}
\hline $\begin{array}{l}\text { Responses at } \\
\text { Uretest and Postte }\end{array}$ & & $\begin{array}{c}\text { Intervention } \\
N(\%)\end{array}$ & & $\begin{array}{l}\text { Control } \\
N(\%)\end{array}$ \\
\hline Current use $^{c}$ & 379) & & & \\
\hline Pretest & Posttest & & & \\
\hline Don't use & Don't use & $191(91.83)$ & 141 & $(82.46)$ \\
\hline & Sometimes use & $7 \quad(3.37)$ & 13 & $(7.60)$ \\
\hline & Regular use & $4 \quad(1.92)$ & 3 & $(1.75)$ \\
\hline Sometimes use & Don't use & $0 \quad(0.00)$ & 3 & $(1.75)$ \\
\hline & Sometimes use & $3(1.44)$ & 2 & $(1.17)$ \\
\hline & Regular use & $1 \quad(0.48)$ & 3 & $(1.75)$ \\
\hline Regular use & Don't use & $0 \quad(0.00)$ & 1 & $(0.58)$ \\
\hline & Sometimes use & $2(0.96)$ & 1 & $(0.58)$ \\
\hline & Regular use & $0 \quad(0.00)$ & 4 & $(2.34)$ \\
\hline
\end{tabular}

Chewed within 24 hours? $(N=403)$

\begin{tabular}{|c|c|c|c|}
\hline Pretest & Posttest & & \\
\hline \multirow[t]{2}{*}{ Yes } & Yes & $(0.90)$ & $5 \quad(2.76)$ \\
\hline & No & $3 \quad(1.35)$ & $(2.76)$ \\
\hline \multirow[t]{2}{*}{ No } & Yes & $6 \quad(2.70)$ & $9(4.97)$ \\
\hline & No & $211(95.05)$ & $162(89.50)$ \\
\hline
\end{tabular}

Are you a regular user? $(N=402)$

\begin{tabular}{|c|c|c|c|}
\hline Pretest & Posttest & & \\
\hline \multirow[t]{3}{*}{ Yes } & Yes & $1(0.45)$ & $2(1.11)$ \\
\hline & No & $4 \quad(1.80)$ & 2 (1.11) \\
\hline & Unsure & $0 \quad(0.00)$ & $1 \quad(0.56)$ \\
\hline \multirow[t]{3}{*}{ No } & Yes & $1(0.45)$ & $5 \quad(2.78)$ \\
\hline & No & $188(84.68)$ & $148(82.22)$ \\
\hline & Unsure & $7 \quad(3.15)$ & 11 (6.11) \\
\hline \multirow[t]{3}{*}{ Unsure } & Yes & (1.35) & $1(0.56)$ \\
\hline & No & $14 \quad(6.31)$ & $7 \quad(3.89)$ \\
\hline & Unsure & $4(1.80)$ & $3(1.67)$ \\
\hline
\end{tabular}

"These data are based on preliminary analysis and include data only from years 1 and 2.

b Fach p value is the result of a chi-square test of significance for all pretest/posttest responses to the question. NS denotes the test was not statistically significant at alpha $=0.05$.

" "Don't use" is defined as "never chewed or dipped," "used only once," or "used more than once but quit." "Sometimes use" is defined as "sometimes, but not every week." "Regular use" is defined as "at least once very week," or "at least once every day." 
TABLE 5: Self-Reported Intention to Use Smokeless Tobacco by Seventh Graders Who Participated in the Pathways to Health Project, 1992 to 1995

\begin{tabular}{|c|c|c|c|c|c|}
\hline $\begin{array}{l}\text { Responses } \\
\text { Pretest an }\end{array}$ & & $\begin{array}{r}\text { Inter } \\
\Lambda\end{array}$ & $\begin{array}{l}\text { ention } \\
(\%)\end{array}$ & & $\begin{array}{l}\text { ontrol } \\
V(0 ; 5)\end{array}$ \\
\hline If best & ered, wo & 401) & & & \\
\hline Pretest & Posttest & & & & \\
\hline Yes & Yes & 4 & $(1.80)$ & 7 & $(3.91)$ \\
\hline & No & 5 & $(2.25)$ & 0 & $(0.00)$ \\
\hline & Unsure & 4 & $(1.80)$ & 2 & (1.12) \\
\hline No & Yes & 1 & $(0.45)$ & 8 & $(4.47)$ \\
\hline & No & 128 & (57.66) & 77 & $(43.02)$ \\
\hline & Unsure & & $13.06)$ & 32 & (17.88) \\
\hline Unsure & Yes & 7 & $(3.15)$ & 9 & $(5.03)$ \\
\hline & No & 22 & $(9.91)$ & 16 & $(8.94)$ \\
\hline & Unsure & 22 & $(9.91)$ & 28 & $(15.64)$ \\
\hline
\end{tabular}

Do you think you will use?d $(\mathrm{N}=403)$

0.016

Pretest

Yes

No

Unsure

\section{Posttest}

Yes

No

Unsure

Yes

No

Unsure

Yes

$9 \quad(4.05)$

3 (1.35)

4 (1.08)

$4 \quad(1.80)$

$106(47.75)$

$27(12.16)$

11 (4.95)

$34(15.32)$

$24(10.81)$
$P$ Value

a These data are based on preliminary analysis and include dala only from years 1 and 2.

"Each p value is the result of a chi-square test of significance for all pretest/posttest responses to the question.

$N S$ denotes the test was not statistically significant at alpha $=0.05$.

"Unsure" is defined as "maybe" or "don t knou"."

"Yes" is defined as "I already chew or dip" or "I'm sure I will cheu' or dip someday."

"Unsure" is defined as "probably yes" or "probably no."

"No" is defined as "I'm sure I nerier will use.

smokeless tobacco under this circumstance again reported "no" at posttest. Also, fewer of the intervention students changed from "no" to "yes," and more of the control students remained unsure.
Overall, few seventh-grade students thought consistently that they would ever use smokeless tobacco ("yes/yes," Table 5), but a larger proportion of control students continued to think they would use 
or were unsure at posttest. Nearly half ( 48 percent) of the intervention students remained sure they would never use smokeless tobacco, compared with 38 percent in the control group.

The proportion of students who reported use of smokeless tobacco increased from pretest to posttest for both the intervention and control groups (intervention group: pretest 3 percent, posttest 8 percent: control group: pretest 8 percent, posttest 15 percent). However, the increase in use from pretest to posttest was significantly greater for control students. This trend was not observed among fifth graders.

\section{Cigarette Smoking}

No statistically significant differences in pretest/ posttest change categories were found among fifth graders' self-reports of smoking and intention to smoke, so these data are not presented in a table format. More than three-fourths (83 percent; 326 divided by 392) of the students in the intervention and control groups reported not smoking at both pretest and posttest, and more than 88 percent ( 377 divided by 428 ) indicated they had not smoked within 24 hours of either test.

A greater percentage of fifth-grade intervention students were certain they would not smoke if their best friend offered them a cigarette ( 4 4 percent of intervention vs. 30 percent of control students), although the results were not statistically significant $(p=0.11)$, and similar results were reported to the question of whether they thought they would ever smoke (31 percent of intervention students answered "no" vs. 14 percent of control students; $p$ $=0.15$ ). Overall, significantly more fifth graders reported smoking sometimes or regularly at the posttest compared with the pretest $(p<0.001)$. Six percent were current smokers at the pretest and 15 percent at the posttest. This trend was observed in both intervention and control groups and in response to potential use if a best friend offered.

Among seventh graders, there were several significant differences from pretest to posttest in recent smoking behavior and likelihood of smoking in the future; unfortunately, these differences were in an unexpected direction. The intervention students were more likely to have reported smoking within 24 hours of each test (7able 6). In addition, they were more likely to think they will smoke cigarettes eventually, with nearly 25 percent answering "yes" at both pretest and postlest, and 15 percent changing from "unsure" on the pretest to "yes" on the postlest (Table 7). Twenty-one percent of intervention students and 15 percent of control students began to smoke or experiment with smoking between the pretest and posttest (Table 6, "Current use").

Increases in smoking frequency from pretest to posttest were significant for both groups $(\mathrm{p}<0.001$ ). Seventeen percent (63 divided by 365 ) of all seventh-grade students were current smokers (defined as sometimes use or regular use) at pretest and nearly one-third ( 32 percent or 117 divided by 365 ) of students smoked sometimes or regularly at posttest. Increases from pretest to posttest in intention to smoke if a best friend offered also were noted in seventh graders $(\mathrm{p}<0.001)$. At pretest, 14 percent said they would smoke if a best friend offered and 39 percent were unsure, compared with 23 percent and 45 percent, respectively, at posttest.

Future analyses will explore potential differences in grade, gender, tribe, and intervention group in relation to reported tobacco use behavior.

\section{DISCUSSION}

Current cigarette use was approximately twice that of smokeless tobacco use in fifth graders (approximately 14 percent vs. 7 percent at posttest). Seventhgrade intervention and control groups also reported greater cigarette use. The self-reported prevalence of tobacco use is consistent with data from other studies of Native American youth $(9,16,17,40,44)$.

of concern, seventh-grade intervention students reported higher levels of cigarette use than control group students at posttest (38 percent "regular" or "sometimes" use vs. 25 percent). Because schools were the unit of randomization, the intervention group may have included schools with stronger environmental factors for initiating smoking or higher prevalences of smoking within those communities. Also, by seventh grade, some American Indian boys have begun to participate in ceremonial activities that include traditional tobacco use. It remains to be clarified if this higher reported use among intervention students is due 10 a misclassification of ceremonial tobacco use with "store-bought" cigarette use.

Self-reported use of cigarettes and smokeless tobacco was higher among seventh graders when compared with fifth graders. In addition, strong trends exist in increased self-reported tobacco use 
TABLE 6: Self-Reported Smoking Behavior Among Seventh Graders Who Participated in the Pathways to Heaith Project, 1992 to 1995

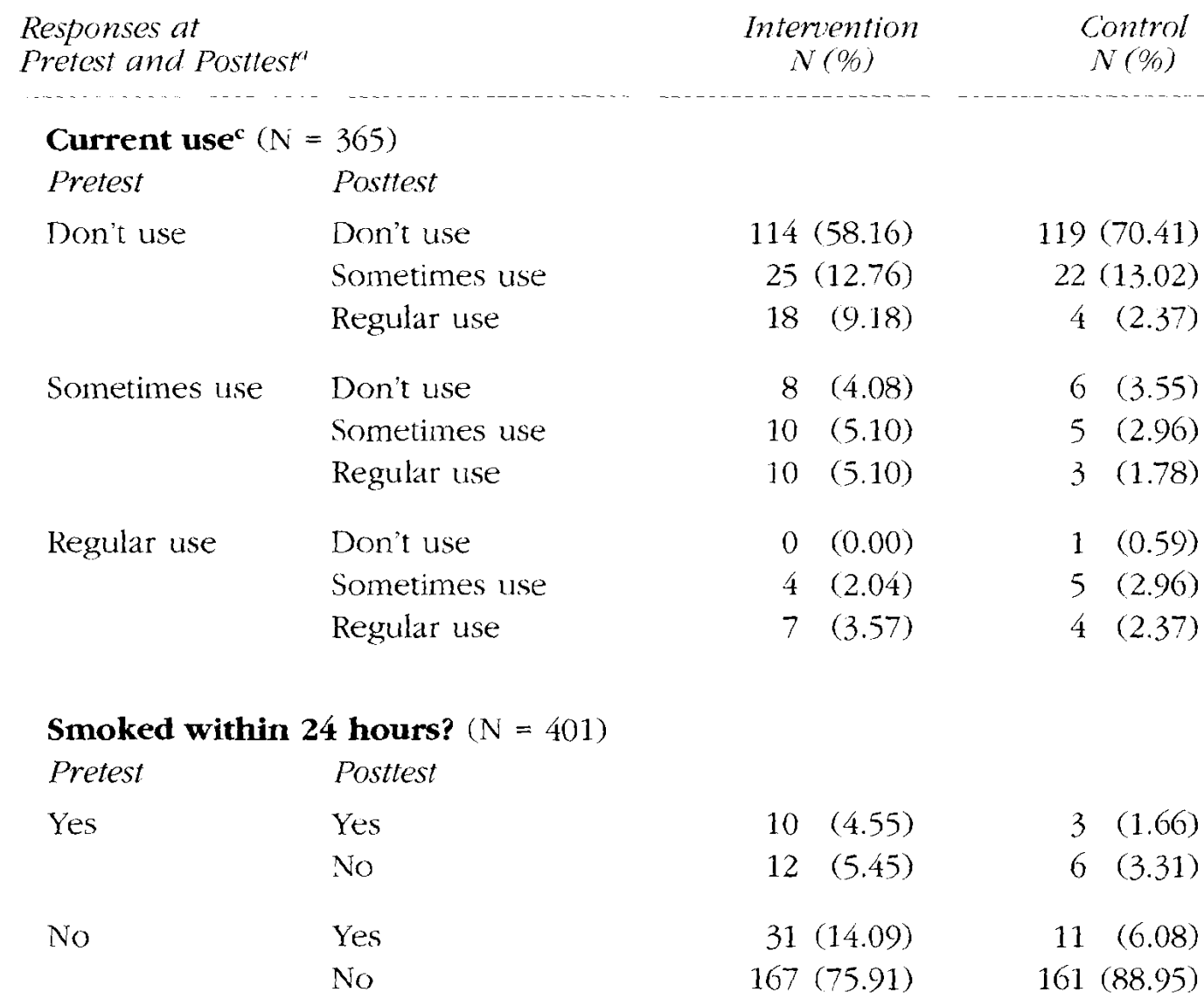

Are you a regular smoker? $(\mathrm{N}=402)$

NS

Pretest

Yes

No $\quad \begin{aligned} & \text { Yes } \\ & \text { No } \\ & \text { Unsure }\end{aligned}$

Unsure
Posttest

Yes

No

Unsure

Unsure

Yes

No

Unsure
$5 \quad(2.26)$

$4 \quad(1.81)$

5 (2.26)

$5 \quad(2.26)$

$126(57.01)$

$31(14.03)$

$7 \quad(3.17)$

$20 \quad(9.05)$

18 (8.14)
0.008

NS 
TABLE 7: Self-Reported Intention to Smoke Among Seventh Graders Who Participated in the Pathways to Health Project, 1992 to 1995

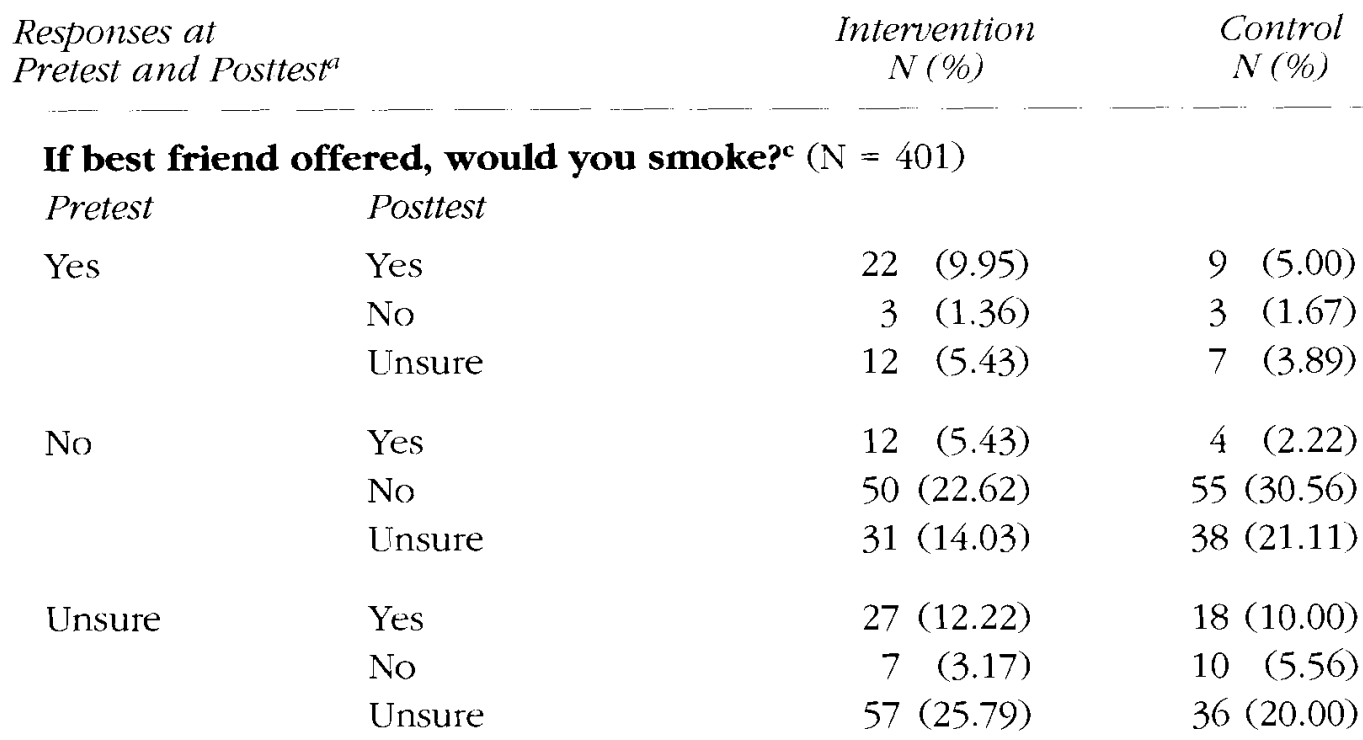

Do you think you will smoke?d $(\mathrm{N}=399)$

0.038

Pretest

Posttest

Yes

Yes

$54(24.55)$

$28(15.64)$

No

$7 \quad(3.18)$

2 (1.12)

Unsure

$15 \quad(6.82)$

16 (8.94)

No

Yes

$15 \quad(6.82)$

$17 \quad(9.50)$

No

29 (13.18)

36 (20.11)

Unsure

25 (11.36)

20 (11.17)

Unsure

Yes

$33(15.00)$

$14 \quad(7.82)$

No

$15 \quad(6.82)$

$17 \quad(9.50)$

Unsure

27 (12.27)

29 (16.20)

"These data are based on preliminary analysis and include data only from years 1 and 2.

${ }^{n}$ Each p value is the result of a chi-square test of significance for all pretest/posttest responses to the question. NS denotes the test was not statistically significant at alpha $=0.05$.

"Unsure" is defined as "maybe" or "don't know."

" "Yes" is defined as "I already smoke" or "I'm sure I uill smoke someday."

"Lnsure" is defined as "probably yes" or "probably no."

"No" is defined as "I'm sure I never will smoke."

and intention to use from pretest to posttest, particularly in the seventh grade. This suggests that children in these grade levels (age 10 to 14 ) may be in a critical transitioning period when experimentation with tobacco products is common. Future health education efforts focused intensively on these 
grades could be extremely useful in reducing behavioral intentions to start tobacco use.

Potential limitations exist for getting accurate self-reports of tobacco use. Underreporting of use by students may have occurred because of their concern that teachers or parents may learn of their answers. During the completion of these surveys, the project staff assured students of the privacy of their responses. Another concern was the accuracy of students' reports or recall of past use of tobacco products. Because the questions were worded in terms familiar to the students and the surveys were administered by the program staff rather than teachers, we believe the results are reasonably accurate.

Lessons learned by this research team are described below:

- Research and service activities in an American Indian community, and especially in a school, must be conducted according to the time schedule of the community. Because of the cross-cultural aspects of research, the intense community interest in all outside-sponsored activities, and the small size and rural nature of reservation communities, research takes considerably longer in an Indian community than in an otherwise similar non-Indian community. The important involvement of the community makes precise prediction of timeframes very difficult.

- Low-fat and high-fiber foods, including fruits and vegetables, may not be available or affordable. The variety of healthful commodity foods distributed by the Federal Government also may be limited. Therefore, planning a healthful diet may be extremely difficult.

- A participatory approach allowed ample opportunities for participating communities to contribute to the creation of a program that supported healthier lifestyles reflecting those in the tribes' traditional cultures. This approach was also realistic economically and geographically. The rich cultural heritage of American Indians, which includes many nutritious low-fat and highfiber foods and healthy lifestyle practices, contributed important concepts to Pathways to Health. Incorporating traditional approaches to learning new behaviors also added value to the curriculum as well as acceptance by the participating American Indian communities.

- It is critical to include a diverse group of community members to advise and consult regarding the appropriateness of using traditional stories, foods, and activities. In many Indian communities, some beliefs and customs are not to be shared in a public venue.

- The prerogatives of the tribal government and local laws and customs must be respected. For example, some tribes have their own research review boards and their own approval processes for informed consent and for manuscript and abstract review. These must be strictly followed.

One of the purposes of Pathways to Health and other research activities that followed was to encourage and support communication between university health educators and American Indian tribes in this region on cancer prevention and control issues. Results from a survey of tribes in New Mexico indicate a strong interest in primary prevention efforts, cancer prevention and control training, barriers to cancer research and treatment, and cancer risk factors for American Indians (45). Therefore, the process of the development, implementation, and evaluation of the Pathways to Health project may be useful to other health promotion researchers in their quest for developing programs most applicable to their audience. The program was very positively received by the communities and schools in which it was implemented. The families that participated in the family component of Pathways to Health were extremely positive in their comments about the project. The need for continued cancer prevention efforts with tribes of the Southwest is apparent. Future cancer prevention and health promotion efforts should extend beyond the schools to incorporate the extended family and the community in intervention activities.

\section{REFERENCES}

1. Cancer control objectives for the nation: 19852000. Natl Cancer Inst Monogr 1986;2:1-93.

2. Committee on Diet and Health, Food, and Nutrition Board, Commission on Life Sciences, National Research Council. Diet and health: implications for reducing chronic disease risk. Washington, DC: National Academy Press, 1989. 
3. Wynder EL, Gori GB. Contribution of the environment to cancer incidence: an epidemiologic exercise. J Natl Cancer Inst 1977;58:825-32.

4. Butrum RR, Clifford CK, Lanza E. NCI dietary guidelines: rationale. Am J Clin Nutr 1988;48(3 Suppl):888-95.

5. Nixon DW. Nutrition and cancer: American Cancer Society guidelines, programs, and initiatives. CA Cancer J Clin 1990;40:71-5.

6. Welty TK. Health implications of obesity in American Indians and Alaska Natives. Am J Clin Nutr 1991;53(6 Suppl):1616S-20S.

7. Valway SE, editor. Cancer mortality among Native Americans in the United States: regional differences in Indian health, 1984-1988, and trends over time, 1968-1987. Washington, DC: U.S. Department of Health and Human Services, Public Health Service, Indian Health Service, 1991.

8. U.S. Department of Health and Human Services. Report of the Secretary's Task Force on Black and Minority Health. Volume I: Executive summary. Washington, DC: U.S. Government Printing Office, 1986.

9. Harris MB, Ford VL. Tobacco use in a fifth-grade Southwestern sample. J Early Adolesc 1988;8:8396.

10. Centers for Disease Control. Preventing tobacco use among young people: a report of the Surgeon General. Executive summary. Mor Mortal Wkly Rep 1994;43:1-10.

11. Glover ED, O'Brien K, Holbert D. Prevalence of smokeless tobacco use in Pitt County, North Carolina. Int J Addict 1987;22:557-65.

12. Boyd G. Use of smokeless tobacco among children and adolescents in the United States. Prev Med 1987;16:402-21.

13. Tomar SL, Giovino GA. Incidence and predictors of smokeless tobacco use among U.S. youth. Am J Public Health 1998;88:20-6.

14. Riley WT, Barenie JT, Mabe PA, Myers DR. Smokeless tobacco use in adolescent females: prevalence and psychosocial factors among racial/ethnic groups. J Behav Med 1990;13:20720.
15. Schinke SP, Gilchrist LD, Schilling RF, Walker RD, Locklear VS, Kitajima E. Smokeless tobacco use among Native Americans and other adolescents in the Northwest. Am J Public Health 1986;78:1051-2.

16. Schinke SP, Schilling RF, Gilchrist LD, Ashby MR, Kitajima E. Native youth and smokeless tobacco: prevalence rates, gender differences, and descriptive characteristics. Natl Cancer Inst Monogr 1989;8:39-42.

17. Hall RL, Dexter D. Smokeless tobacco use and attitudes toward smokeless tobacco among Native Americans and other adolescents in the Northwest. Am J Public Health 1988;78:1586-8.

18. Public Health Service. Indian Health Service. Regional differences in Indian health. Rockville, MD: U.S. Department of Health and Human Services, Public Health Service, 1990.

19. Story M, Strauss KF, Zephier E, Broussard BA. Nutritional concerns in American Indian and Alaska Native children: transitions and future directions. J Am Diet Assoc 1998;98:170-6.

20. Broussard BA, Johnson A, Himes JH, Story M, Fichtner R, Hauck F, et al. Prevalence of obesity in American Indians and Alaska Natives. Am J Clin Nutr 1991;53(6 Suppl):1535S-42S.

21. Heyward VH, Harris MB. Physical characteristics related to coronary heart disease risk factors: comparison of Hispanics and Navajo, Acoma, and Laguna Indians in New Mexico. Am J Health Promot 1988;3:25-32.

22. Sugarman IR, White LL, Gilbert TJ. Evidence for a secular change in obesity, height, and weight among Navajo Indian schoolchildren. Am J Clin Nutr 1990;52:960-6.

23. Kimm SY, Gergen PJ, Malloy M, Dresser C, Carroll M. Dietary patterns of U.S. children: implications for disease prevention. Prev Med 1990;19:432-42.

24. Allensworth DD, Kolbe LJ. The comprehensive school health program: exploring an expanded concept. J Sch Health 1987;57:409-12.

25. Jackson MY, Broussard BA. Cultural challenges in nutrition education among American Indians. Diabetes Educ 1987;13:47-50. 
26. Hughes CK, Aluli NE. A culturally sensitive approach to health education for Native Hawaiians. J Health Educ 1991;22:387-90.

27. Reid R, Davis SM. Research in American Indian communities: implications for change. Am J Clin Nutr 1998 (in press).

28. Johns-Steiner V. Learning styles among Pueblo children: final report. Report to National Institute of Education, U.S. Department of Health, Education, and Welfare. Albuquerque: University of New Mexico Press, 1975.

29. More AJ. Native Indian learning styles: a review for researchers and teachers. I Am Indian Educ 1987;26:17-29.

30. Havinghurst RJ, Korol M, Pratt IE. Environment and the draw-a-man test: the performance of Indian children. J Abnorm Psychol Soc Psychol 1946; 40:50-63.

31. Rhodes RW. Nurturing learning in Native American students. Hotevilla, AZ: Sonwai Books, 1994.

32. Lee Little Soldier. Working with Native American children. Young Children 1992;47:15-21.

33. Swisher K, Deylule D. The styles of learning are different, but the teaching is just the same: suggestions for teachers of American Indian Youth. J Am Indian Educ 1989;28:28-32.

34. Gilliland $H$. Teaching the Native American. Dubuque, IA: Kenclall/Hunt Publishing Company, 1992.

35. Harris MB, Davis SM, Ford VL, Tso H. The checkerboard cardiovascular curriculum: a culturally oriented program. J Sch Health 1988; 58:104-7.

36. Davis SM, Lambert LC, Gonez Y, Skipper B. Southwest Cardiovascular Curriculum Project: study findings for American Indian elementary students. J Health Educ 1995;26(2 Suppl):S72S81.

37. Davis SM. General guidelines for an effective and culturally sensitive approach to health education. In: Matiella AC, editor. The multicultural challenge in health education. Santa Cruz, CA: ETR Associates, 1994:117-32.
38. Perry CL, Baranowski T, Parcel G. How individuals, environments, and health behavior interact: social learning theory. In: Glanz K, Lewis FM, Rimer B, editors. Health behavior and health education: theory, research, and practice. San Francisco: Jossey-Bass, 1990:161-86.

39. Doak CC, Doak LG, Root JH. Teaching patients with low literacy skills. Philadelphia: JB Lippincott Company, 1985.

40. Davis SM, Lambert LC, Cunningham-Sabo L, Skipper B. Tobacco use: baseline results from Pathways to Health, a school-based project for Southwestern American Indian youth. Prev Med 1995:24:454-60.

41. Cunningham-Sabo L, Davis SM. Pathways to Health: a health promotion and cancer prevention project for American Indian youtl. Alaska Med 1993;35:275-8, 296.

42. Cunningham-Sabo L, Davis SM, Koehler KM, Fugate ML, Ditucci JA, Skipper BI. Food preferences, practices, and cancer-related food and nutrition knowledge of Southwestern American Indian youth. Cancer 1996;78(7 Suppl):1617-22.

43. Hall GE, Hord SM. Change in schools-facilitating the process. Albany, NY: State University of New York Press, 1987.

44. Bruerd B. Smokeless tobacco use among Native American school children. Public Health Rep 1990;105:196-201.

45. Osborn KL, Davis SM, Slattery M, Giuliano A, Teufel NI, Joe J, et al. Four Corners research consortium for Native Americans and cancer research. Cancer 1996;78(7 Suppl):1629-32.

\section{ACKNOWLEDGMENTS}

This study was supported by Grant No. U01CA52283 from the National Cancer Institute, 1990 to 1995. 


\section{AUTHORS}

\section{Sally M. Davis, Ph.D.}

Director

Center for Health Promotion and Disease Prevention

Associate Professor

School of Medicine

Health Sciences Center

University of New Mexico

Albuquerque, NM 87131-5311

Tel: (505) 272-4462

Fax: (505) 272-4857

E-mail: smdavis@unm.edu

\section{Leslie Cunningham-Sabo, M.S., R.D., CHES}

Research Scientist III

Center for Health Promotion and Disease Prevention

School of Medicine

Health Sciences Center

University of New Mexico

Albuquerque, NM 87131-5311

Tel: (505) 272-4462

Fax: (505) 272-4857

E-mail: lcsabo@unm.edu
Lori C. Lambert, M.A.

Research Scientist III

Center for Health Promotion and Disease Prevention School of Medicine

Health Sciences Center

University of New Mexico

Albuquerque, NM 87131-5311

Tel: (505) 272-4462

Fax: (505) 272-4857

E-mail: llambert@salud.unm.edu 


\title{
Chapter 8 \\ Tobacco Use Prevention and Dietary Modification Among American Indian Youth in the Northeast
}

\author{
Steven Schinke, Kristin Cole, and Beverly Singer
}

\section{ABSTRACT}

$\mathrm{T}$ his paper discusses the development, implementation, and testing of an intervention to reduce cancer risks through tobacco use prevention and dietary modification among American Indian youths in the Northeastern United States. Youths who received both tobacco prevention and dietary modification intervention increased their knowledge of tobacco facts and ceremonial tobacco use, raised their awareness of the motives of tobacco advertising, and showed a greater ability to resist peer pressure between pretest and posttest and between pretest and followup. Youths who received tobacco intervention only had a greater increase in their knowledge of tobacco facts and advertising awareness than youths in the control group at followup.

As for dietary variables, youths in the combined intervention also increased their knowledge of American Indian dietary practices and of cancer risks relative to fat, fiber, and fruits and vegetables at followup. Youths who received dietary modification intervention only had a greater increase in their knowledge of American Indian dietary practices and of the protective effects of a diet rich in fruits and vegetables at followup than youths in the control group. The combined tobacco use prevention and dietary modification intervention appears to have had the most success in influencing youths away from tobacco use and toward a more healthy diet.

\section{INTRODUCTION}

American Indians have a high prevalence of cancers associated with behavioral and lifestyle patterns (1-3). Theory-based, culturally sensitive, and empirically tested strategies to prevent cancer among American Indians are needed. Especially needed are strategies to reduce cancer risk for American Indian children and adolescents. Because of their ability to make early lifestyle changes, American Indian youths have much to gain from preventive interventions.
To advance the science of cancer risk reduction interventions for younger American Indians, this chapter presents the results of a study of the efficacy of an intervention to prevent tobacco use and modify dietary habits among American Indian youths in the Northeastern United States. The chapter begins by describing the process of selecting and then collaborating with each site for the cancer prevention study. Next, we outline the curriculum and describe the intervention process and delivery of the curriculum at each intervention site. Finally, data 
from field-testing of the intervention curriculum are presented and discussed.

\section{CURRICULA}

\section{Tobacco Use Prevention Curriculum}

We developed the tobacco prevention curriculum after extensive reviews of the literature (1.4-14). Data on the knowledge, attitudes, and beliefs of American Indian youths about risk-taking, collected via self-report procedures, helped to shape the intervention. These data also helped us to determine the appropriate level and complexity of intervention instruction. In addition, because collaborating community representatives made it clear that building youth awareness of their cultural background was essential to the success of the program, we incorporated such cultural information into each curriculum lesson.

Using an educational and skills-based approach, the tobacco use prevention curriculum addressed the multiple stresses associated with such forces as tobacco marketing strategies and peer pressure for young people to begin smoking cigarettes or using smokeless tobacco. In 15 sessions, youths learned skills in resistance, self-control, decisionmaking, problemsolving, and self-reward. To add to the program's sensitivity, the curriculum incorporated material describing traditional ceremonial and cultural uses of tobacco in American Indian societies.

Youths learned facts about smokeless and smoked tobacco use via films, storytelling, and other activities. They collected newspaper and magazine advertisements for both smokeless and smoked tobacco. Under the supervision of the group leader, youths critiqued these advertisements for accuracy, realism, and motivational purposes. This critique helped youths to see how the mass media heavily promote lifestyle habits. Study youths also investigated stereotypes of American Indian life in television, pop culture, books, and films.

Problemsolving skills enabled youths to examine their options and make reasonable decisions when faced with urges, temptations, and pressures to use tobacco. Using the acronym SODAS, youths practiced effective responses to peer and other pressures in rok-plays. Youths were coached to Stop, consider their Options, make a Decision, and Act. Self-praise, the last step in the SODAS model, encouraged youths to praise themselves for thoughtful problemsolving.

\section{Dietary Modification Curriculum}

Like the tobacco curriculum, the dietary modification intervention emphasized the role of media in influencing behavior. Intervention required youths to critique such advertisements as magazine and billboard images that promote unhealthy life choices to eat popular "fast foods." Intervention sessions also stressed traditional heliefs and practices of American Indians concerning respect for one's self, family, and community. Youths were encouraged to respect their bodies' requirement for healthy foods. Finally, throughout the curriculum, youths learned and practiced resistance skills, decisionmaking, problemsolving, and self-reward skills to help them resist pressures to eat poorly and to enhance their self-efficacy.

\section{Tobacco Use Prevention and Dietary Modification Curriculum}

For the combined intervention, we selected eight tobacco use prevention sessions and seven dietary modification sessions. Because many sessions in each curriculum shared similar content regarding life skills (e.g., decisionmaking, problemsolving) and cultural identification, we chose sessions for inclusion in the intervention that focused on tobacco or diet, respectively, and that did not repeat content from other sessions.

\section{METHODS}

A randomized clinical trial, the research design included four conditions: tobacco use prevention and dietary modification combined, tobacco use prevention alone, dietary modification alone, and nointervention control. The study took place in seven different sites, located in Connecticut, Maine, Massachusetts, New Hampshire, New Jersey, New York, and Rhode Island, with representation from 50 different tribal affiliations. Randomly, by coin toss, two sites received dietary intervention only (D), two sites received tobacco intervention only (T), two sites received tohacco and diet intervention combined (T\&D), and two sites served as control groups.

Recruitment interviews with participating community sites led us to believe that the collaborating 
American Indian agencies served sufficient numbers of American Indian youths in our target age range. We later discovered that services at many of these sites were sporadic and typically geared to adults rather than children. Because no regular recreational or social activities for children were offered, families with children were sometimes unacquainted with the participating health and human services' organizations and thus leery of participating in our intervention. For other families, transportation to and from a participating center was an obstacle to participation. Although American Indian organizations reported accurately the number of Native American youth they serve annually, the numbers shrink and swell depending on the time of year. Many of the American Indians served by our urban sites spend considerable time each year on their native reservations and thus were unavailable at various times throughout intervention delivery.

Thus, despite our initial plans to target 250 American Indian youths, recruitment yielded only 150 youths. One T\&D site withdrew from the study before the pretest, resulting in a study sample comprised of 135 youths ( 57.7 percent female), ages 8 to 13 at pretest, with youths approximately evenly distributed across conditions except for the T\&D group, which had fewer youths. By posttest, 96 youths $(54$ percent female) remained in the study, and at followup, 86 youths ( 55 percent female) completed outcome measurements. All youths identified themselves as Native American or American Indian. Collaborating group leaders and group leader assistants recruited youths. Most youths were fifth graders, with approximately similar numbers enrolled in fourth, sixth, and seventh grades. Youths participated in the study for 3 years.

\section{Procedure}

Onsite, youths in all of the intervention groups completed pretest, posttest, and followup measures at the beginning of, the end of, and 6 months after the intervention period. Youths self-reported dietary and tobacco habits, knowledge and attitudes toward tobacco use and diet, and food frequencies. Youths in the control group completed the same pretest, posttest, and followup batteries and, 8 months after the conclusion of the intervention period, received a 2-day health and nutrition intervention.
After pretesting, youths received intervention in 15 one-hour weekly group sessions from pairs of group leaders. Group leaders were prepared to deliver the curriculum to youths in a total of 20 hours of training in a workshop format. Together with pretest and posttest sessions, study youths participated in a total of 17 sessions, each approximately 50 minutes in length. Sessions were held after the school day or concurrent with regularly scheduled recreational activities at the participating community centers.

\section{Format}

Beginning with the first session, youths were directed toward healthy choices. American Indian heritage is made manifest in such Native-oriented projects as making story bags, dance sticks, and sacred hoops. American Indian storytellers shared their cultural knowledge and answered questions about ancestral life. Other role models included visual artists and theater performers. Via films, lectures, and activities, youths learned facts about smokeless and smoked tobacco use and about food and nutrition. Youths receiving dietary intervention only were encouraged to eat a variety of foods and to reduce their fat intake by consuming more whole grains, beans, and fresh fruits and vegetables as sources of healthy fiber. Youths also learned how others influence their dietary and tobacco use behavior. Study youths were asked to sign a contract with themselves to try a new food or to change a negative eating habit.

At the intervention's conclusion, youths in the intervention arms invited their families to a program in which they shared their intervention experiences and education. At every site, family participation was excellent. Parents, aunts and uncles, grandparents, and siblings of youth attended the sessions. Project staff awarded a certificate to every youth who completed the intervention.

\section{Process Measurement}

Process measures assessed the reliability of intervention delivery, provided ongoing data to increase within-arm consistency, and provided feedback on strengths and weaknesses of intervention content and activities. The research staff observed 33 percent of all intervention sessions in all conditions. During these observations, the staff recorded the 
leaders' adherence to intervention protocol, the youths' participation in intervention, and the extent to which leaders interacted with youths.

Leaders summarized every intervention session on a standard form, recording the youths' contributions, problems, and matters needing remedy. Summary data helped to maintain the focus of each intervention. To quantify confounding sources of intervention, we gave the youths a contamination measure at posttest and at all followup sessions. The measure asked youths whether and in what form they received information about tobacco use, dietary habits, and cancer-related or health promotion content other than that provided in the study.

\section{Outcome Measurement}

Pretest data on youths' dietary and tobacco habits were gathered via self-report procedures (12-14). Youths responded to multiple-choice questions regarding the prevalence and number of cigarettes smoked, smokeless tobacco used, alcoholic beverages drunk, and drugs consumed during the index reporting period (usually the most recent preceding day and week). They also completed the Block food frequency survey (15). Other questions gathered information on the youths' demographic characteristics, knowledge and attitudes about tobacco and their diets, education level, self-esteem, communication skills, problemsolving skills, and other psychosocial determinants of tobacco use and diet. Saliva sampling with subsequent cotinine analyses to validate self-reports of tobacco use among youths in the tobacco intervention-only group was attempted without success. Youths in the tobacco and diet intervention group completed both the Block food frequency survey and the tobacco use prevention survey, as did youths in the control group.

\section{RESULTS}

\section{Process Findings}

Across conditions, staff members were pleased with group leaders' adherence to intervention protocol and the extent to which the leaders interacted with youths. As for youths' participation in intervention sessions, leaders noted that reservation youths were less inclined to participate in theater games, music, and physical activities than their urban counterparts. Generally, reservation youths were more active par- ticipants in group activities than in those activities that required their independent action. Youths across sites appeared highly engaged in storytelling activities and in demonstrations (e.g., making mayonnaise, demonstrating smoking machines).

Leaders recommended devoting the initial intervention session to introductions rather than pretests. They felt that youths needed time to get comfortable with each other and to feel safe and valued. Leaders also felt that giving tests in the first session biased some youths against the intervention. In one site, for example, three youths did not report to the second session. When the leader pursued these children, they reported that they were "turned off" by the testing. Fortunately, the leader was able to convince the youths to come back. Giving the pretests in the second session, according to the group leaders, would be less threatening for the youths.

Group leaders agreed with research staff that youths preferred active group efforts to activities that "put them on the spot." Also, although the leaders were unanimously enthusiastic about the merits of the final community presentation session, leaders reported that they were forced to use too much of the last few sessions to prepare youths for their skits and presentations. Leaders suggested adding more community presentation sessions or more family involvement in general and adding at least one extra session for preparation time per each community or family session.

Finally, leaders described difficulties in getting youths to complete posttest and followup surveys. Two leaders reported that youths resented filling out the same questionnaire that they completed in pretest. Leaders recommended spending more time at pretest explaining to youths the significance of the surveys. They also recommended shortening the surveys as much as possible.

\section{Outcome Findings}

The authors analyzed data by means of one-way analysis of variance (ANOVA) to determine omnibus levels of significance among the study's four arms. Then, when $F$ ratios revealed significance, Scheffé $a$ posteriori tests were conducted to compare means of individual arms.

To reiterate, the study sample comprised 135 youths ( 57.7 percent female) at pretest, age 8 to 13 (mean age $=9.88$ years, $\mathrm{SD}=1.27$ ). All youths iden- 
tified themselves as Amcrican Indian or Native American. Most youths were fifth graders $(n=62)$, with approximately similar numbers enrolled in fourth $(n=23)$, sixth $(n=36)$, and seventh $(n=13)$ grades. The research design involved a nested sample, in which youths were members of groups that were assigned to study arms. Thus, comparisons of outcome differences used youths as the unit of analysis, even though a smaller sample of groups was involved.
Although pretest scores failed to differ among the four study conditions, ANOVA comparisons of posttest and followup gain scores revealed differences on several outcome variables. (Gain scores were computed by subtracting pretest scores from posttest scores.) Youths' mean gain scores on tobacco use variables indicated improvements coincident with intervention delivery. Shown in Figure 1, outcome measurement scores for ceremonial (traditional) tobacco use among American Indians showed

FIGURE I: Mean Knowledge and Attitude Outcome Measurement Scores for All

\section{Conditions}

$\square$ Pretest (N=135) $\quad$ Posttest (N=96) Followup $(\mathrm{N}=86)$
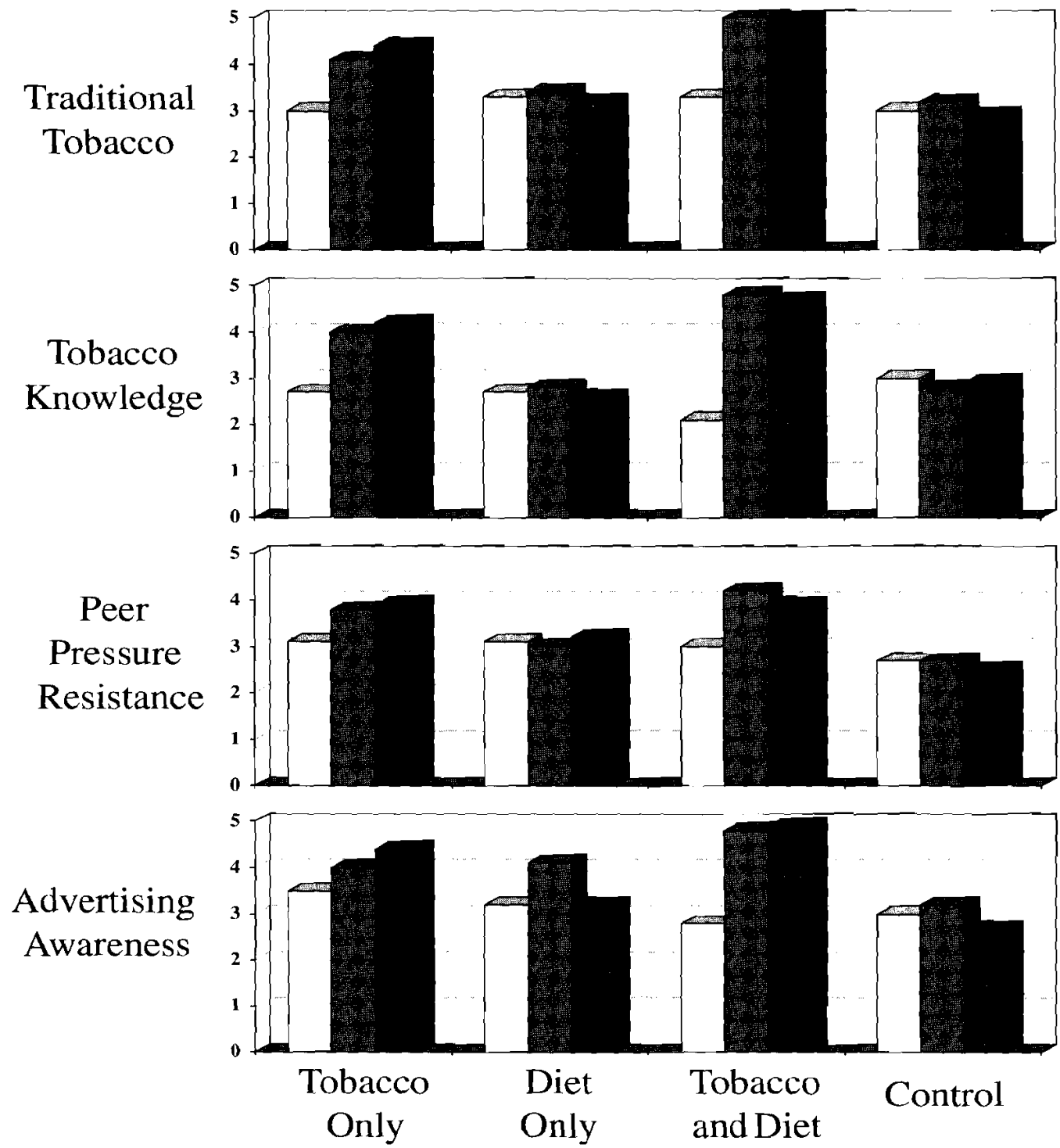
pretest-to-posttest differences $(\mathrm{F}[3,92]=5.01, \mathrm{p}<.01)$. On this variable, youths in the T\&D group reported greater knowledge than youths in the $\mathrm{T}, \mathrm{D}$, or control group, by Scheffé post boc comparisons of gain score means. Knowledge of the negative effects of smoking and of smokeless tobacco use differed between pretest and posttest $(\mathrm{F}[3,92]=6.71, \mathrm{p}<.001)$.

According to Scheffe comparisons, youths in the $T$ and $T \& D$ groups knew more about tobacco at posttest than their counterparts in the $\mathrm{D}$ and control groups. Differences were evident between pretest and posttest on the variable measuring youths' ability to resist peer pressure to use tobacco $(F[3,92]=$ $5.98, \mathrm{p}<.001$ ). Youths in the T\&D and $\mathrm{T}$ groups reported more ability for such resistance than did youths in the $D$ and control groups, according to Scheffé post boc tests. Youths' awareness of the objectives and methods of advertising differed at posttest $(F[3,92]=6.13, p<.001)$. Scheffé post boc comparisons of means (at the $\mathrm{p}<.05$ significance

FIGURE 1: Mean Knowledge and Attitude Outcome Measurement Scores for All Conditions (continued)

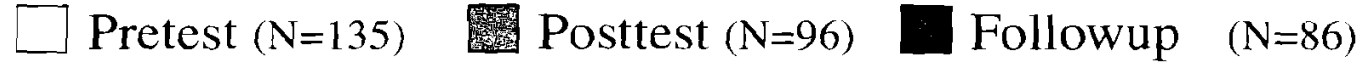

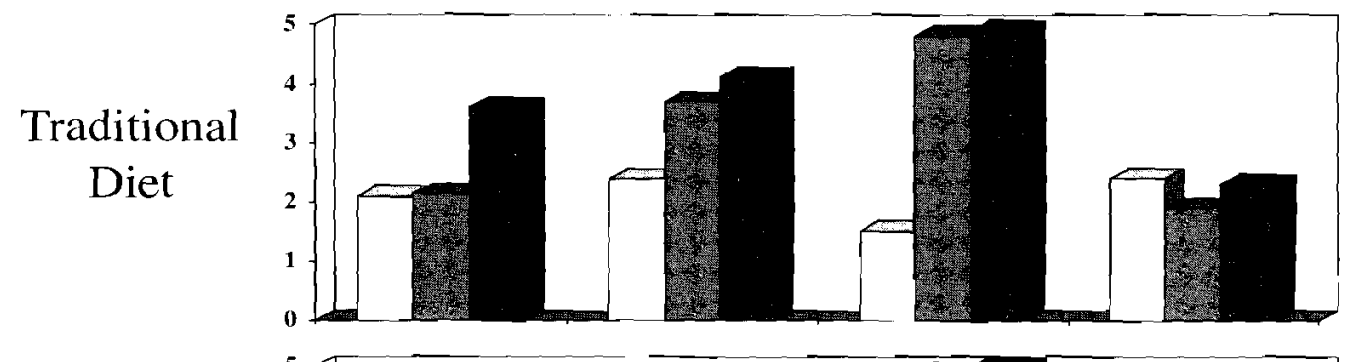

Fat

Knowledge

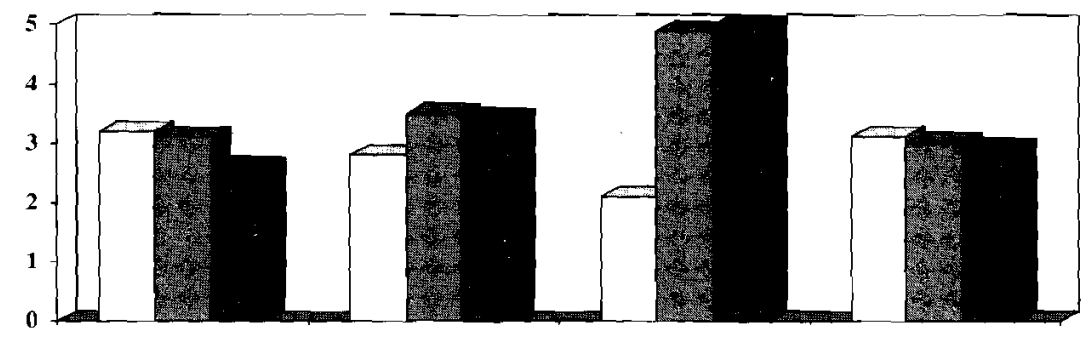

Fiber

Knowledge

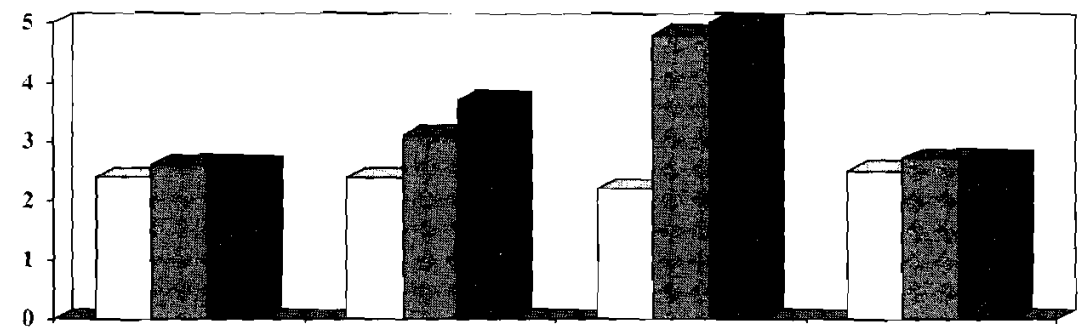

Fruits and

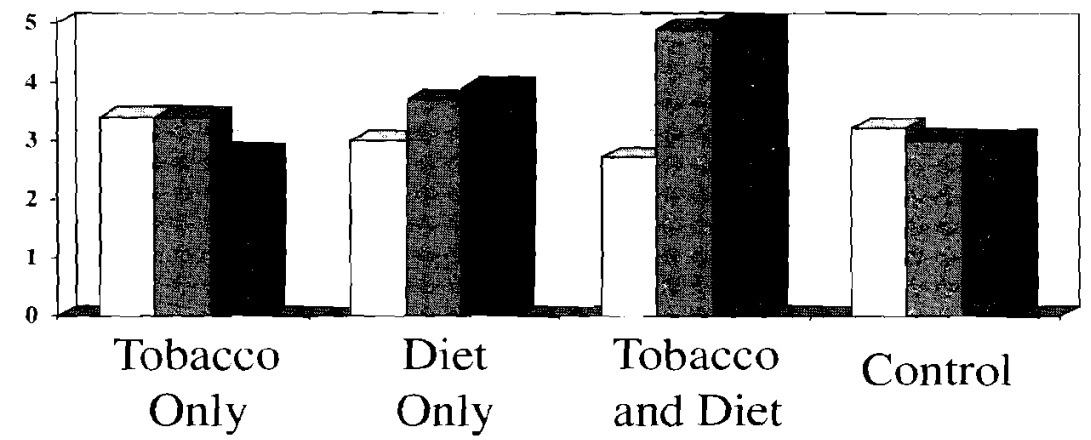


level) indicated that these differences on the advertising variable favored the T\&D and D conditions over the control condition.

On dietary knowledge variables, analyses of pretest-to-posttest findings revealed a number of differences. Knowledge of American Indian dietary traditions differed among conditions from the pretest to the posttest measurement $(\mathrm{F}[3,92]=10.72, \mathrm{p}<$ .001 ), in favor of T\&D and $\mathrm{D}$ youth relative to $\mathrm{T}$ and control youth, according to Scheffé comparisons ( $\mathrm{p}$ $<.05$ ). At posttest, knowledge of cancer risks related to the consumption of fat $(F[3,92]=3.92, p<.01)$, fiber $(F[3,92]=6.62, p<.001)$, and fruits and vegetables $(F[3,92]=9.62, p<.001)$ differed among conditions relative to the pretest occasion. Scheffé comparisons indicated that youths in the T\&D group had a greater increase in their knowledge of cancer risks associated with fat, fiber, and fruits and vegetables than youth in the T, D, and control groups.

Differences among conditions were apparent also at followup. Knowledge of traditional tobacco use in American Indian culture differed among conditions at followup measurement minus the pretest score (F $[3,82]=8.69, p<.001)$. Scheffé post hoc comparisons of means (at the $\mathrm{p}<.05$ significance level) indicated that the T\&D group scored higher than both the $\mathrm{D}$ and control groups, whereas the $\mathrm{T}$ group scored higher than the control group only. On the variable measuring knowledge of the negative effects of tobacco, differences also were evident among conditions $(F[3,82]=11.34, p<.001)$. According to Scheffé comparisons, the T\&D and T conditions had greater knowledge of the negative effects of tobacco than youths in the $\mathrm{D}$ and control conditions. Responses to the item assessing youths' perceived ability to resist peer pressure also differed between pretest and followup $(\mathrm{F}[3,82]=6.61, \mathrm{p}<.001)$. Youths in the T\&D and T groups scored higher at followup than youths in the control group, according to Scheffé, on the variable assessing peer pressure resistance. Outcome measurement scores on the variable for advertising awareness differed among conditions at followup relative to pretest $(\mathrm{F}[3,82]=12.83, \mathrm{p}<.001)$. Scheffé comparisons indicated that youths in the T\&D and $T$ groups scored higher on advertising awareness than youths in the $\mathrm{D}$ and control groups.

Followup measurements revealed differences among conditions in responses to dietary variables.
Knowledge of American Indian dietary traditions differed between pretest and followup measurements among conditions $(F[3,82]=11.92, p<.001)$. All three intervention conditions scored higher (with T\&D scoring the highest) on this variable compared with youths in the control condition, according to Scheffé comparisons. At followup, differences were apparent in knowledge of fat $(\mathrm{F}[3,82]=5.02, \mathrm{p}<$ $.01)$, fiber $(F[3,82]=5.51, p<.01)$, and fruits and vegetables $(F[3,82]=14.60, p<.001)$. Scheffé comparisons indicate that for the variables measuring knowledge of fat and fiber, youths in the T\&D groups scored better than youths in the $\mathrm{D}$, $\mathrm{T}$, or control groups; however, for the variable measuring knowledge of fruits and vegetables, youths in the T\&D and D groups scored higher than youths in the $T$ or control groups.

\section{CONCLUSION}

Data yielded by this longitudinal study reveal patterns of improved knowledge and attitudes relative to tobacco use and dietary habits among American Indian youths who were exposed to the cancer risk reduction curriculum. In particular on tobacco use questions, pretest-to-followup measurement scores for youths in the T\&D group reveal increased knowledge of American Indian tobacco traditions, increased knowledge of the harmful effects of smoked and smokeless tobacco use, greater awareness of the motives of tobacco advertising, and higher ratings of the ability to resist peer pressure following participation in the cancer risk reduction curriculum compared to all other groups. On the variables measuring knowledge of the harmful effects of tobacco and peer pressure resistance, youths in the $T$ group increased their scores more than youths in the $D$ and control groups. For variables measuring knowledge of ceremonial tobacco use and advertising awareness, youths in the $T$ group scored better than youths in the $\mathrm{D}$ and control groups.

As for dietary variables, pretest-to-followup measurement scores show that after receiving the curriculum, T\&D youths improved their knowledge of the health implications of consuming dietary fat, fiber, and fruits and vegetables as well as their knowledge of traditional American Indian diets more than youths in the other groups. For the variable measuring knowledge of traditional American Indian diet, 
youths in all three intervention groups showed improvement compared to youths in the control group. Youths who received D intervention only knew more about fruits and vegetables at followup than youths in the $\mathrm{T}$ and control groups, but not as much as youths who received the T\&D curriculum.

The study is not without weaknesses. Because randomization was at the site and not at the individual level, subjects were necessarily nested in the design. Therefore, outcome findings could have occurred in part from the independence of subjects within sites. The relatively small sample size further calls into question the wide-scale generalizability of study outcomes. Still, and in spite of these weaknesses, the study allows modest conclusions about intervention effects.

Overall, the combined tobacco use prevention and dietary modification intervention appears to have had the most success in influencing youths away from tobacco use and toward a more healthy diet.

Process data also revealed directions for future research. Session summary data suggest that intervention arm youths were engaged with the majority of sessions throughout delivery. Yet, leaders noted their difficulties in teaching the content in only 15 sessions. As a result of these time constraints, leaders felt that intervention delivery did not fulfill the ambitions of the intervention. Leaders advised adding 15 more sessions and lengthening each session to 2 hours. Additional and longer sessions would allow the youths to better internalize the material. In particular, the leaders observed that youths learned best when they had more sessions in which to review and practice skills. Thus, skills learned in earlier sessions were more evident to group leaders than skills learned later in the program.

That measurement changes coincided with youths' receipt of the curriculum directed at tobacco use prevention and dietary modification lends credence to the suggestion that the curriculum in part accounted for pretest-to-followup improvements in intervention youths' knowledge, attitudes, perceptions, and behavioral intentions. Yet conclusions about the effectiveness of the respective interventions are seriously limited by the paucity of data on behavioral change among the study sample. Therefore, the present findings must be considered merely suggestive of the promise of the curriculum.
Such promise derives from the positive findings between measurement occasions on youths who received the $T \& D$ intervention curriculum and from the culturally sensitive nature of the intervention program and the manner in which it was implemented. The curriculum represented an initial attempt to construct cancer risk reduction intervention for American Indian youths that addresses both tobacco use and dietary practices. It combined elements of past preventive programs with elements particular to American Indian youths. In so doing, the intervention joins theory-based and empirically tested approaches to behavior change with culturally sensitive content that at once mirrors and respects American Indian traditions around tobacco use and food choices. Such a blending of science with cultural sensitivity represents a novel approach to cancer risk reduction with American Indian youths.

\section{REFERENCES}

1. Blum RW, Harmon B, Harris L, Bergeisen L, Resnick MD. American Indian-Alaska Native youth health. JAMA 1992;267:1637-44.

2. U.S. Department of Health and Human Services. Cancer mortality among American Indians in the United States. Washington, DC: 1991.

3. Lanier AP. Epidemiology of cancer in Alaska Natives. Alaska Med 1993;35:245-7.

4. Beauvais F. An integrated model for prevention and treatment of drug abuse among American Indian youth. J Addict Dis 1992;11:63-80.

5. Beauvais F, LaBoueff $B$. Drug and alcohol abuse intervention in American Indian communities. Int J Addict 1985;20:139-71.

6. Cvetkovich G, Earle TC, Schinke SP, Gilchrist LD, Trimble JE. Child and adolescent drug use: a judgment and information processing perspective to health-behavior interventions. J Drug Educ 1987;17:295-313.

7. Hall RL, Dexter D. Smokeless tobacco use among American Indians and other adolescents in the Northwest. Am J Public Health 1988; 78:1586-8.

8. Kendall PC. Cognitive-behavioral therapies with youth: guiding theory, current status, and emerging developments. J Consult Clin Psychol 1993;61:235-47. 
9. Oetting ER, Beauvais F, Edwards R, Waters MR, Velarde J, Goldstein GS. Drug use among Native American youth: a summary of findings (19751981). Fort Collins, CO: Colorado State University, 1983.

10. Resnick H, Wojcicki M. Reaching and retaining high risk youth and their parents in prevention programs. In: Goplerud E, editor. Preventing adolescent drug use: from theory to practice. OSAP Prevention Monograph 8. DHHS Publication No. ADM 91-1725. Rockville, MD: Office of Substance Abuse Prevention, 1991:91126.

11. National Center for Chronic Disease Prevention and Health Promotion, Office on Smoking and Health. Preventing tobacco use among young people: a report of the Surgeon General. Atlanta: U.S. Department of Health and Human Services, Public Health Service, Centers for Disease Control and Prevention, 1994.

12. Contento I, Michela JL, Goldberg CJ. Food choice among adolescents: population segmentation by motivations. J Nutr Educ 1988;20:28998.

13. Contento I, Roberts S, Gussow ID. Using adolescents' dietary self-reports to tailor schoolbased nutrition education. J Nutr Educ 1986;18:175-81.

14. Murray DM, O'Connell CM, Schmid LA, Perry $\mathrm{CL}$. The validity of smoking self-reports by adolescents: a reexamination of the bogus pipeline procedure. Addict Behav 1987;12:7-15.

15. Block G, Hartman AM, Naughton D. A reduced dietary questionnaire: development and validation. Bethesda, MD: Division of Cancer Prevention and Control, National Cancer Institute, and Epidemiology Resources, Inc., 1990.

\section{ACKNOWLEDGMENT}

Research repotted in this paper was sponsored by Grant No. U01CA52251 from the National Cancer Institute.

\section{AUTHORS}

Steven Schinke, Ph.D.

Columbia University School of Social Work 622 West 113th Street

New York, NY 10025

Tel: (212) 898-9339

Fax: (212) 361-2209

E-mail: schinke@walrus.com

\section{Kristin Cole, M.S.}

Columbia Liniversity School of Social Work 622 West 113th Street

New York, NY 10025

Tel: (212) 898-9334

Fax: (212) 361-2209

E-mail: kristin@walrus.com

\section{Beverly Singer, Ph.D.}

Columbia University School of Social Work 622 West 113th Street

New York, NY 10025

Tel: (212) 898-9334

Fax: (212) 361-2209

E-mail: tewabrs@aol.com 


\title{
The National Cancer Institute's Research Efforts in Native American Communities: Approaches Used and Lessons Learned
}

\author{
Felicia Schanche Hodge and Claudia Sanchez Glover
}

\section{ABSTRACT}

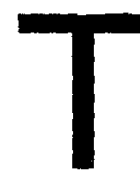

o provide culturally acceptable, accessible, and appropriate cancer interventions, researchers must identify those approaches to cancer prevention and control that work and those that do not work. To explore this issue in Native American communities, we reviewed the major health intervention approaches employed by the National Cancer Institute's (NCl's) Native American research projects. This chapter discusses these approaches and highlights their similarities and differences. The authors suggest that effective interventions among Native people will require customizing the intervention to the specific target group. It is important to use culturally acceptable and sensitive approaches in the planning, design, and implementation phases to develop an intervention model that is well received and effective in Native communities.

\section{INTRODUCTION}

In 1989, the NCl supported and funded a collaboration of cancer research projects targeting American Indians, Alaska Natives, and Native Hawaiians in various sites across the United States. These projects highlighted major approaches to designing and implementing cancer control programs for Native communities. The major goal of the projects was to provide strategic policy or intervention approaches that would be culturally acceptable and sensitive to Native populations. Although these models are not the only available intervention models for use in Native communities, they are important because of their success and should be considercd appropriate interventions for future research. In this chapter, the methodological approaches used by these projects in the areas of tobacco control, nutrition, and interventions for cancers of the breast and cervix arc identified and discussed.
These projects were located throughout the United States in Alaska, California, Hawaii, Idaho, New Mexico, New York, North Carolina, Oregon, and Washington. The projects were located in urban and rural areas in tribal settings, Native communities, schools, and health clinics. They focused on early detection and prevention of specific cancers, teaching youth and adults about the health threats of tobacco use and seeking ways to change behaviors known to increase cancer risk significantly.

The NCI-supported interventions described in this monograph represent a turning point in cancer prevention and control for Native Americans. Cultural sensitivity was a hallmark of these projects. Native American researchers, staff, advisors, and community leaders were significantly involved in the projects, which enhanced their success. Furthermore, the interventions were adapted to the cultural dynamics of Native Americans, accomplished in part by the 
use of focus groups during the planning stages in several of the projects. For example, the Ilawaii project used focus groups to help identify culturally appropriate interventions. Participants were asked to identify concerns about cancer and barriers to cancer screening. When asked, "How could we do a better job of researching cancer control in Hawaiian women?" the participants responded that small group meetings, such as focus groups, were ideal for identifying barriers and acceptable solutions. Local Native Hawaiian communities endorsed the use of navigators, or local Native women, to assist in promoting good health and to recommend appropriate screening behaviors to further promote acceptable healthcare.

The kokua group approach developed for the intervention encourages sharing experiences and discussing barriers to screening and overcoming barriers. The concept, kokua, is defined as a mutual willingness to assist without an expectation of return and without having to be asked. The women received up-to-date information about cancer prevention and control. The kokua groups provided a safe haven where cultural identity and history were valued. Women could feel comfortable within the groups using traditional Hawaiian concepts, understanding, and language.

These NCI projects have helped researchers acquire a better understanding of Native populations and their cultures. The smoking cessation project in California adapted a proven, physician-based smoking cessation model to health clinics serving Native people. The researchers found that a high level of awareness of the harmful effects of tobacco had litthe impact on smoking-related attitudes and behavior because of cultural factors. Traditionally, American Indians are reluctant to interfere with the behavior of others. This promotes more lenient attitudes and behaviors toward smoking. Respect for the rights of others is an important social value. For example, it is often culturally unacceptable to ask others not to smoke, even in one's own home. Thus, smoking control programs for Native Americans must develop effective methods to address these cultural attitudes. Although smoking rates are declining in the general population, this project found that 40 percent of adult American Indians in northern California are smokers, almost twice the rate in the general U.S. population. Nearly 60 percent of Indian males and 50 percent of Indian females in San Francisco smoke (1).

The Southwest project significantly expanded our knowledge about cancer risk behaviors among Native youth. Based in New Mexico, this investigation documented cancer-related nutrition knowledge among fifth- and seventh-grade American Indian students. In addition, the researchers found that Native boys and girls are experimenting with tobacco. Findings also suggest that the sixth grade may be a critical transition period for intervention. The Northeast project, based in New York, also focused on youths and showed the importance of using an integrated approach, featuring tobacco prevention and a dietary modification intervention, instead of an isolated approach for promoting overall health and well-being.

These projects suggest that culturally sensitive interventions can make a difference in influencing cancer-related behaviors. This was evident in the Alaska study where, during the 3 years prior to the intervention, only 12 percent of Alaskan women surveyed knew how to reduce their risk of cervical cancer. By the end of the study, 79 percent of the Native Alaskan women surveyed were aware of cervical cancer screening and the importance of following up on medical recommendations (2).

The tobacco policy project in the Pacific Northwest (Washington, Oregon, and ldaho) used another innovative approach to cancer control: it sought to revise American Indian tribal policies regarding smoking and using smokeless tobacco. This project resulted in a significant increase in more stringent indoor smoking policies among the 39 tribes participating in the study. The tribes consistently reported that the newly implemented smoking policies were enforced (with most smoking occurring outdoors at the tribal meetings) and that the nosmoking policy was respected.

Another strength of these projects is the use of effective research methods, which enable the researchers to better assess the impact of their interventions. The North Carolina study used a Solomon Four-Group Design. This approach allows researchers to rule out the effect of pretesting and to detect changes in behavior that might be occurring over time both with and without the intervention. 
The California group used a control group in a longitudinal study design. This allowed for tracking of the smokers' behavior over time in a controlled setting. Also, the California project used Prochaska's stages of readiness $(3,4)$ to measure the smokers' stage of readiness and to identify other variables that predict smoking status at 6 and 18 months. Other projects administered pretest and posttest surveys by using instruments such as the Likert scale (5) and theoretical models such as the health belief model (6).

\section{RESEARCH INTERVENTION APPROACHES}

Few studies address cancer control intervention models in Native American communities, and even fewer document those that are successful in reaching the project's goals (7). To address this need, those research projects and interventions that are effective in Native American communities must be identified. The NCI Native American research projects reviewed in this monograph can be used as building blocks for further innovative research that will directly address the health needs of Native populations

Table 1 illustrates the four major intervention approaches used in the design of the cancer prevention and control projects in Native populations. These approaches are identified as policy change, educational change, behavioral change, and replication of intervention models. Although these approaches are not mutually exclusive, they illus- trate the different methods used in the design and implementation of the projects.

The following section highlights important categories and approaches that were fundamental to the projects.

\section{Policy Change}

Policy change is defined as an overall plan embracing general goals and acceptable procedures, especially of a governmental body (8). Policy change is often the most difficult approach because it takes a longer period of time to implement and to measure results. Although harder to achieve, policy change often has longer lasting results. The Northwest project implemented a tobacco policy approach that affected tobacco control in the area of restricting abusive smoking practices. This program also took advantage of community involvement and advocacy for public smoking restrictions with tribes in Idaho, Washington, and Oregon. The Northwest project successfully targeted tobacco policy changes aimed at restricting abusive smoking behavior in tribal facilities and in tribal community gatherings.

Tobacco has had a long and important history among many tribes. Revered for its spiritual and ceremonial use, tobacco was used for trade with early colonists. Today, tribes continue to use tobacco ceremonially and to benefit economically from the tobacco industry (9). Thus, implementing an intervention that examines and changes longstanding tobacco practices involves educating the Indian

\section{TABLE I: Research Intervention Approaches in Native Populations}

Project

\section{Approaches}

Policy

Educational

Behavioral

Replication

$\begin{array}{ll}\text { Alaska } & \mathrm{X} \\ \text { North Carolina } & \mathrm{X} \\ \text { Hawaii } & \mathrm{X} \\ \text { Northwest } & \\ \text { California } & \mathrm{X} \\ \text { Southwest } & \mathrm{X} \\ \text { Northeast } & \mathrm{X}\end{array}$


community and tribal counsels on the benefits of the policy change. It also involves working with the community to develop and implement the changes. The resulting policy, setting up smoke-free tribal areas, was identified as instrumental in setting the stage for future programs of smoking cessation and tobacco control that were successful in Native communities.

\section{Educational Change}

Educational change includes those teachings that increase an individual's level of knowledge. The educational interventions presented in this monograph are similar in their use of role models along with educational material to promote positive change. In this approach, respected peers or community members often serve as role models to promote positive behavior and to enhance the acceptance of educational material. Testimonials are often the basis for the presentation. Various media approaches-video, television, radio, tape recordings, and printed materials-also are used to enhance education and knowledge.

The projects in Alaska, North Carolina, the Northwest, and the Southwest used educational intervention models in their prevention programs. The Alaska project designed cervical cancer educational materials with illustrations of Native Alaskan stylized dancers; these informational reading materials detailed when and where to get a Pap test. The materials included information on where and why Native women should seek cancer screening. Likewise, the North Carolina project developed educational materials in a format acceptable and culturally sensitive to the target population.

A smoking cessation videotape, It's Your Life, highlighted testimony by American Indians and Alaska Natives on the negative effects of smoking. The video played a significant role in the California project, providing the motivational support to quit smoking. A self-help guide, emphasizing quitting methods ("slow turtle" or the "cold turkey"), accompanied the video. A video produced by the Southwest project played a significant part in presenting the cancer prevention message to both students and their families. This video, Life in Balance, includes the testimony of six American Indian adults who are regarded as role models in their communi- ties, describing their personal journeys to reach a more healthful balance in their lives through healthy lifestyle choices.

Peer education, provider training, and community involvement were the overarching strategies in both the tobacco projects and the cervical cancer projects. The Native community easily identified and accepted the health information as culturally appropriate and important. To further involve the community, the projects employed Native community members to present the educational materials.

\section{Behavioral Change}

Modifying the response or action of an individual or group is the result of behavioral change. The strategy used by the Hawaii project incorporated designing and implementing a culturally sensitive intervention as a means of increasing breast and cervical cancer screening practices, thus changing behaviors. The kokua group is the name of the primary intervention created for this project. The project used traditional Hawaiian values and communication patterns as the basis for an educational curriculum that promotes breast and cervical cancer screening among Native I Hawaiian women. Positively changing the hehavior of Native Hawaiian women in cancer screening took place through the diffusion of information and support that occurred in the community. This model incorporated traditional Hawaiian practices-i.e., the health navigator leading Native Hawaiian women to the project and assisting them in promoting their well-being through appropriate health screening examinations.

\section{Replication of Intervention Models}

Replicating tried and true intervention models is a common approach to addressing community health needs. Rather than "reinventing the wheel," this approach identifies projects and interventions that were reported as successful in a cultural setting. Project staff then adapt all or portions of the model to fit the specific needs of the community. Refinement or reassessment of the model is sometimes necessary, especially when working with Native communities, to make the intervention culturally appropriate and accessible.

The California project was based on NCI's stopsmoking model. This NCI model trained physicians 
and other providers to counsel patients to quit smoking and to monitor and assist them in that decision (10). Important to the program was the establishment of clinic protocols for recordkeeping, for patient monitoring of the stages of cessation, and for improving patient appointment-keeping and involvement in health care.

The Indian clinics are often seen as the focus of Indian communities in California. Because this population identified smoking cessation as a priority for research, the research team was able to adapt an NCI smoking model to the Indian community by involving Indian clinic providers and Indian health workers from the community. Using the strength of the Native community by subcontracting Indian health staff, such as community health representatives, and revising the smoking cessation message to include important cultural values, beliefs, and cues to action, became important aspects of adapting the model. The design and content of the smoking cessation materials were tailored to the Native community.

\section{SIMILARITIES IN APPROACHES}

Overall, the strategies used by the NCI Native American projects were similar in that they all used the common approach of "working with the community" in the design and implementation of the projects. Generally, the strategy undertaken was as follows:

- Identify the problem and design the intervention with the Native community;

- Hire Native staff or subcontract a portion of the work with Native organizations;

- Use community resources; and

- Use focus groups to design project materials and questionnaires to ensure cultural appropriateness, sensitivity, and acceptability.

\section{Working With the Community in Problem Identification and Design}

The first step undertaken by all projects was working on a collaborative basis with the targeted community representatives to identify the barriers and to design the appropriate intervention. Obtaining agreement from the Native communities to implement a community-based program in the targeted area was essential in gaining access to the commu- nities. Several projects obtained written memoranda of agreements (or understandings) and resolutions of support from the Native communities. These agreements were obtained at the beginning of the projects or during the design and writing of the proposal. The research team provided presentations of the proposed projects, and input was obtained from the tribe, tribal counsel, health board, or other Native agency.

\section{Staffing Patterns of the Research Team}

Conducting research among Native people requires an understanding of the cultural milieu of the Native community as well as the trust of the community and project members. This understanding and trust can be obtained by securing a staffing pattern that includes Natives on the project and in the frontline staffing (those staff with immediate contact with the community).

The research teams of each of the NCI projects comprised principal investigators and research scientists with experience in American Indian, Alaska Native, and/or Native Hawaiian community interventions. Although only two of the projects had American Indian principal investigators, all had frontline personnel who were Native. The project directors and support staff were primarily Native Americans.

Incorporating a team that reflects the community dynamics, instills trust, and assists in the development of culturally appropriate and acceptable materials is essential to a successful program. Survey design and questionnaire development benefitted from the multicultural team approach as the project incorporated differences in language, concepts, and approaches to community needs and concerns.

\section{Use of Community Resources}

A rich reserve of community resources is often available to the research team. These resources include community gatekeepers, elders, libraries, and storytellers. Community members and tribal staff are often available to be trained and employed as survey workers. Tribal counsels, tribal leaders, and health advisory boards are excellent resources to be used as sounding boards and to provide direction in relations with communities. 


\section{Incorporating Cultural Beliefs in the Interventions}

Focus groups composed of community members can assist in the identification of important goals and the development of culturally appropriate interventions. To provide a successful project for the Native community, the research team must be knowledgeable about the culture and sensitive to acceptable modes of delivery. Incorporating cultural beliefs through the use of role models, educational materials, and an emphasis on community involvement proved to be a successful intervention technique. Capturing the cultural dynamics, with group educational sessions, media and educational materials, and interpersonal exchanges proved to be effective in cancer control projects.

The Southwest project incorporated many cultural beliefs and values-such as learning through observation and practice, learning from storytelling, and learning cooperatively-in its school-based curriculum. Elders also were included as teachers in the classroom, and they taught the students (often in their native language) about how traditional practices and values relate to healthy lifestyle practices of today.

All the projects were careful to incorporate culturally sensitive styles and appropriate language in the print and video materials. Native artwork was commonly used, and educational messages incorporated cultural concepts including the importance of family and cooperation. The Hawaiian project used the cultural concept of kokua, or mutual assistance, cooperation, and helping one another without being asked (e.g., use of the navigator). These kokua groups consisted of small groups of women connected by bonds of friendship or family. The women in the kokua groups shared their knowledge and experience about cancer and supported each other in using breast cancer screening techniques, breast self-exams, and mammograms.

The California project used the cultural message of quitting smoking for yourself, your community, and your tribe. The New York project used artwork and American Indian stories to enhance the delivery of the project's message.

\section{DIFFERENCES IN APPROACHES USED}

Important differences existed among the projects in the NCI Native American research experience.
These differences include the following:

- educational approaches;

- group size differences;

- geographic locations;

- site variations; and

- demographic characteristics.

\section{Use of a Structured Educational Curriculum vs. Unstructured Approach}

Several projects used structured curricula for training purposes and as a means of providing standard instruction. The Southwest project developed and implemented a structured cancer prevention curriculum taught by classroom teachers for their fifthand seventh-grade students. The Northeast project also used a tobacco use prevention curriculum to address the multiple stresses associated with cigarette smoking and smokeless tobacco use among Indian youth. The curricula were well-received and instrumental in providing accessible and acceptable health information.

Several projects incorporated Native or unstructured approaches in their instruction. The most obvious example was the Hawaiian project, which incorporated the traditional Hawaiian concept of kokua, or mutual cooperation, and the use of a navigator to assist in implementing the educational intervention. The California project incorporated local Native concepts of responsibility to family, tribe, and community in its educational media messages. The Southwest project incorporated Native foods, traditional uses of tobacco, and the importance of community in its design. The Northeast project incorporated material describing ceremonial and cultural uses of tobacco in American Indian societies.

\section{Individual vs. Group Approaches}

Several interventions targeted individuals; others targeted large or small groups. Cervical and breast cancer education projects, such as those in Alaska and North Carolina, tended to target inclividuals for the delivery of the message and counseling. The smoking cessation project design in California incorporated a one-on-one message from the provider to the smoker with followup by community health representatives. Interventions using larger group sessions 
included the Northwest policy intervention project and the Southwest school-based intervention. Others preferred smaller, more intimate group designs.

\section{Differences in Regional Groups}

The cultural differences seen among Alaska Natives, Native Hawaiians, and various American Indian tribes were evident in the languages, cultural beliefs, and health or illness belicfs exhibited among the groups. Researchers also noted differences between the urban and rural groups in the interventions. These differences were related to socioeconomic levels, education, and employment.

The Hawaiian group responded well to the use of the kokua, which is rooted in their traditional culture. Alaska Native and American Indian groups also responded positively to information that was tailored by design and content to their culture. Availability of project materials at the Indian clinic sites was important and essential to improving access to health information.

\section{Location of Intervention Activities}

The locations of intervention activities varied considerably among the projects. The projects were located as far as Alaska to the north, New Mexico to the south, New York and North Carolina to the east, and the Pacific Northwest with California and Hawaii to the west. Within the regional sites, project locations differed by activity on reservations, tribal trust lands, and urban sites and within Native "community" groups. The interventions took place in schools, health clinics, tribal or Indian agency offices, and other facilities.

\section{Demographic Characteristics}

The most obvious demographic differences among the projects were the age and sex of groups targeted for the intervention. Projects targeting women's health issues, such as breast and cervical cancers, restricted their target population to women age 18 and older. Tobacco control and nutrition projects included both male and female participants. The tobacco and nutrition projects, located in the Northeast and the Southwest regions, targeted only adolescents in their study populations.

\section{CONCLUSION}

A review of the NCI Native American research projects identified four major intervention approaches: policy change, educational change, behavioral change, and replication of current intervention models that achieved favorable results in Native communities. The similarities and differences among these approaches were examined, and the steps taken within each research project for achieving successful community collaboration and project implementation were documented by the authors.

In designing an appropriate cancer intervention project, researchers must consider those approaches to cancer prevention and control that work and those that are culturally appropriate and acceptable to Native communities. The authors suggest that effective interventions in Native communities should be customized to the specific culture of the target population. Working within the Native community in the planning, design, and implementation phases will produce more effective and acceptable interventions and lead to success in completing project goals.

This monograph has moved our efforts closer to a clearer understanding of successful cancer prevention and control interventions among American Indians, Alaska Natives, and Native Hawaiians. Nevertheless, there continues to be an overwhelming need to continue research efforts in the areas of tobacco control and cessation, nutrition, and breast and cervical cancers.

The four primary prevention projects targeting tobacco control and cessation and nutrition provided significant information on the role of culture, both as a facilitator and as a barrier to smoking cessation and control. The Northwest project demonstrated the success of working within the culture of the target population, developing and implementing policies at the tribal level. The California project also worked with the Native population in providing a culturally sensitive intervention. However, the American Indian traditional values of noninterference and respect for independence were shown to promote more lenient attitudes and behaviors toward smoking. The Southwest and Northeast projects expanded our knowledge about cancer risk behaviors among Native youth. These projects documented early experimentation with smoking and 
recommended the importance of using an integrated approach, targeting tobacco and diet, to promote overall health and well-being. Given that the highest smoking prevalence is reported among American Indians and Alaska Natives in comparison to nonIndians (11), and that smoking is one of the most preventable risk behaviors for cancer, interventions of this nature are paramount in improving the health of Native populations

Among all the projects, cultural sensitivity in the planning, implementation, and evaluation of projects was significant to their success. Cervical cancer is a serious health concern in all Native communities because of high morbidity and poor 5-year survival rates (12). Breast cancer rates, which are very high among Native Hawaiians and Alaska Natives, are of particular concern (13). These projects demonstrated their innovative approach to health education and prevention intervention, showing that culturally sensitive interventions can make a difference in influencing cancer risk behaviors. The Alaska project tailored educational materials on cervical cancer specifically to the Alaska Native culture and included Alaska Natives in the planning and implementation of the project. The North Carolina project found that using community resources and staff increased the acceptability and credibility of the intervention. The Hawaii project's cultural approach of using a traditional Hawaiian method of group support and interaction was significant to the success of the project. The Hawaii project used kokua groups as the basis for merging cultural identity with the intervention to form support groups receptive to learning and changing behaviors.

This monograph is a tangible product that can be of great use to Native communities. The information from these research projects can lead to more successful program planning and interventions that are more adaptable and acceptable to Native populations. These NCI projects are working models that can be replicated and have a positive effect on the health and well-being of Native American communities.

\section{REFERENCES}

1. Hodge FS, Cummings S, Fredericks L, Kipnis P, Williams M, Teehee K. Prevalence of smoking among adult American Indian clinic users in northern California. Prev Med 1995;24:441-6.
2. Lanier AL, Berner J. Prevention of cervical cancer in Alaska Native women. Final Project Report. Bethesda, MD: National Cancer Institute, Office of Special Populations Research, 1985.

3. Rohren CL, Croghan IT, Hurt RD, Offord KP, Marusic Z, McClain FL. Predicting smoking cessation outcome in a medical center from stage of readiness: contemplation versus action. Prev Med 1994;23:335-44.

4. Prochaska J. Assessing how people change. Cancer 1991;67(3 Suppl):805-7.

5. Rubin A, Babbie E. Research methods for social work. Pacific Grove, CA: Brooks/Cole Publishing Company, 1993:193-4.

6. Gillam SI. Understanding the uptake of cervical cancer screening: the contribution of the health belief model. Br I Gen Pract 1991;41:510-3.

7. LeMaster PL, Connell CM. Health education interventions among Native Americans: a review and analysis. Health Educ Q 1994;21:521-38.

8. Webster's Ninth New Collegiate Dictionary. Merriam-Webster Inc. Springfield, MA: 1995:910.

9. Hodge FS. Tobacco control leadership in American Indian communities. In: Slama K, editor. Tobacco and health. New York: Plenum Press, 1995:375-8.

10. Glynn T, Manley M. How to help your patients stop smoking. A National Cancer Institute manual for physicians. Bethesda, MD: U.S. Department of Health and Human Services, Public Health Service, National Institutes of Health, 1989

11. Shelton DM, Merritt R, Robinson RG. Tobacco control strategies. In: Slama K, editor. Tobacco and health. New York: Plenum Press, 1995:367-74.

12. Baquet $\mathrm{C}$, Ringer $\mathrm{K}$. Cancer among blacks and other minorities: statistical profiles. NIH Publication No. 86-2785. Bethesda, MD: U.S. Department of Health and Human Services, Public Health Service, National Institutes of Health, National Cancer Institute, 1986.

13. Baquet CR. Native Americans' cancer rates in comparison with other peoples of color. Cancer 1996;78(7 Suppl):1538-44. 


\section{AUTHORS}

\section{Felicia Schanche Hodge, Dr.P.H.}

Director

Center for American Indian Research and Education

1918 University Avenue, Suite 2A

Berkeley, CA 94704

Tel: (510) 843-8661

Fax: (510) 843-8611

E-mail: FeliciaSH@aol.com

\section{Claudia Sanchez Glover, M.H.S.}

Health Statistician

Officer of Special Populations Research

National Cancer Institute

Executive Plaza South, Suite 320

9000 Rockville Pike

Bethesda, MD 20892

Tel: (301) 496-8589

Fax: (301) 435-9225

E-mail: cg51c@nih.gov 


\section{Appendix A \\ Glossary}

Age Adjustment: A statistical method used to enable cancer incidence (or mortality) rates to be compared between two populations with different age structures. In this monograph, the 1970 U.S. standard million population was used to calculate the age-adjusted rates found in Chapter 1.

Alpha Error: An error made by rejecting a true null hypothesis (such as claiming that a relationship exists when it does not). Also called Type I Error

Alpha Level: The chance a researcher is willing to take of committing an alpha error or Type I Error; that is, of rejecting a null hypothesis that is true. The probability that a Type I Error (wrongly rejecting the null hypothesis) has been committed. The smaller the alpha level, the more significant the finding because the smaller the chance that the finding is due to chance alone. Thus, an alpha level of .01 is a more difficult criterion to satisfy than a level of .05 . Also called level of (statistical) significance.

Alternative Hypothesis $\left(H_{1}\right.$ or $\left.\mathbf{H}_{\mathbf{a}}\right)$ : In hypothesis testing, any hypothesis alternative to the onc being tested, usually the opposite of the null hypothesis. Also called the research hypothesis. Rejecting the null hypothesis shows that the alternative (or research) hypothesis may be true.

ANOVA: A test of the statistical significance of the differences among the mean scores of two or more groups on one or more variables or factors. It is an extension of the $t$ test, which can only handle two groups, to a larger number of groups. More specifically, it is used for assessing the statistical significance of the relationship between categorical independent variables and continuous dependent variables. The procedure in an analysis of variance (ANOVA) involves computing a ratio (F ratio) of the variance within the groups (error variance) to the variance between the groups (explained variance).
Average Annual Rate: See cancer incidence and mortality rates.

Avoidable Mortality: As used in this monograph, refers to cancer deaths which could be avoided through numerous ways, such as by state-ofthe-art early detection screening programs.

Baseline: An observation or value that represents the normal background level of a measurable quantity, used for comparison with values representing response to experimental intervention or an environmental stimulus, usually implying that the baseline and response values refer to the same individual or system.

Beta Error: An error made by accepting or retain ing a false null hypothesis-more precisely, by failing to reject a false null hypothesis. Also called Type II Error.

Between-Group and Within-Group Differences: Usually contrasted to differences within the groups being studied in an ANOVA. Betweengroup differences are what the researcher is interested in; they are considered large only if they are large in comparison with within-group differences.

Cancer Incidence: The number of new cascs of cancer diagnosed in a specified population during the year.

Cancer Mortality: The number of deaths from cancer in a population during the year.

Cancer Control: The reduction of cancer incidence, morbidity, and mortality through an orderly sequence from research on interventions and their impact in defined populations to the broad systematic application of the research results.

Cancer Incidence and Mortality Rates: The number of new cancers diagnosed in a specified population during a year (incidence rate) and the number of deaths from cancer in a population during a year (mortality rate). Both of these 
rates are presented in this monograph as the number of cancer events (diagnoses or deaths) per 100,000 people. Since cancer diagnoses and deaths are accumulated over 5 years (1988 to 1992) in this monograph in Tables 1 and 2 of Chapter 1 , the cancer incidence and mortality rates are calculated by dividing the number of cancers (new cases or deaths) by five times the 1990 population. The resulting rate is referred to as an average annual rate. In general, incidence rate is the number of new cases that have occurred during a given interval of time divided by the population at risk at the beginning of the time interval. The numerator in a mortality rate is the number of people who died during a given period of time, and the denominator is the number of people who were at risk of dying during the same period.

Carcinogen: Any cancer-causing agent or substance.

Chi Square $\left(\mathbf{X}^{2}\right)$ : A test statistic used to assess the statistical significance of a finding. The most familiar use of the chi-square test occurs when a researcher wants to see if there are statistically significant differences between the observed (or actual) frequencies and the expected (or hypothesized, given the null hypothesis) frequencies of two variables presented in a crosstabulation or contingency table. The larger the observed frequency is in comparison with the expected frequency, the larger the chi-square statistic and the more likely the difference is statistically significant.

Confidence Interval: A range of values of a sample statistic that is likely (at a given level of probability, called a confidence level) to contain a population parameter. The interval that will include the population parameter a ccrtain percentage (confidence level) of the time. The wider the confidence interval, the higher the confidence level.

Confidence Level: A desired percentage of scores (usually $95 \%$ or $99 \%$ ) that would fall within a certain range of confidence limits.

Consultation Intervention: An intervention prompted and assisted by a consultant who is outside an organization (tribe) but where the organization retains primary responsibility for the intervention.
Control Group: In experimental research, a group that, for the sake of comparison, does not receive the treatment the experimenter is interested in

Cooperative Agreement: This assistance mechanism is similar to the traditional National Institutes of Health (NIH) research grant. It differs from a research grant principally in the extent and nature of National Cancer Institute (NCI) programmatic involvement with investigators during performance of the planned activity.

Correlation: The extent to which two or more things are related to one another. This is usually expressed as a correlation coefficient.

Correlation Coefficient: A number showing the degree to which two variables are related. Correlation coefficients range from -1.0 to +1.0 . If there is a perfect negative correlation (-1.0) between $\mathrm{A}$ and $\mathrm{B}$, whenever $\mathrm{A}$ is high, $\mathrm{B}$ is low, and vice versa. If there is a perfect positive correlation $(+1.0)$ between $\mathrm{A}$ and $\mathrm{B}$, whenever one is high or low, so is the other. A correlation coefficient of 0 means that there is no relationship between the variables. (A zero correlation may also occur when two variables are related but their relationship is not linear.)

Degrees of Freedom (df): The number of values free to vary when computing a statistic. This number is necessary to interpret a chi square, or an $\mathrm{F}$ ratio. In a cross tabulation, the df are computed by multiplying the number of rows minus 1 times the number of columns minus 1 ; $\mathrm{df}=(\mathrm{R}-1)(\mathrm{C}-1)$. Thus, the more categories the variables are broken into, the higher the degrees of freedom.

Demographic Profile: A description of a subject's ethnic-racial background, socioeconomic status, age, and gender.

Dependent Variable: The presumed effect in a study, so-called because it "depends" on another variable. It is the variable whose values are predicted by the independent variable, whether or not caused by it.

Dietary Modification: An organized set of activities designed to change a person's diet.

Distribution: A ranking, from lowest to highest, of the values of a variable and the resulting pattern 
of measures or scores, often as these are plotted on a graph.

Education and Skill-Based Approach: An approach that emphasizes information as well as teaching such skills as assertiveness, decisionmaking, and problemsolving.

Empirical Outcome Data: Data gained from an experiment or observation.

Epidemiological Databases: Data collected for and used in the investigation of diseases.

Error: The difference between an observed score and a predicted or estimated score.

Error Term: The part of an equation indicating what is unexplained by the independent variables. The error term specifies how big the unexplained part is.

Error Variance: Any uncontrolled or unexplained variability, such as within-group differences in an ANOVA. The error variance is the variance of the error term.

Experimental Group: The group receiving some treatment in an experiment. Data collected about people in the experimental group are compared with data about people in a control group (who received no treatment) and/or another experimental group (who received a different treatment).

Explained Variance: Variance in the dependent variable that can be accounted for by variance in the independent variable(s).

F Ratio (or Value or Statistic): The ratio of explained to unexplained variance in an analysis of variance; that is, the ratio of the betweengroup variance to the within-group variance. To interpret the $\mathrm{F}$ ratio, you need to consult a table of $F$ values for a particular level of statistical significance at the number of degrees of freedom in your study.

Fiber: The sum of the constituents of the diet that are not digested by gastrointestional enzymes.

Focus Group: Type of research in which a moderator leads about 8 to 10 people in talking freely about a topic.

Gatekeepers: A group of expert individuals who, through their positions of power in a community and/or as representatives of segments of the target audience, can "speak for" the audience.
Health Belief Model: Based on belief that perceived susceptibility to disease or injury influences action toward preventive behaviors. The model attempts to explain and predict healthrelated behavior in terms of certain belief patterns. The model is based on the following assumptions about behavior change: (1) The person must believe that he or she is susceptible. For example, for an asymptomatic disease like hypertension or early stage cancer, the person must believe that he or she can have it and not feel symptoms; (2) the person must perceive the potential seriousness of the condition in terms of pain or discomfort, time lost from work, economic difficulties, and so forth; (3) on assessing the circumstances, the person must believe that benefits stemming from the recommended behavior outweigh the costs and inconvenience and are indeed possible and within his or her grasp; and (4) there must be a "cue to action" or a precipitating force that makes the person feel the need to take action. This last point is fundamental to the entire model. Health education can provide the cue to action if the predisposing factors represented by the health beliefs are correctly diagnosed.

Health Promotion: An organized series of activities which promote healthy living.

Holistic Learning: An integrated approach to learning.

Hypotheses Testing: The classical approach to assessing the statistical significance of findings. Basically, it involves comparing empirically observed sample findings with theoretically expected findings-expected if the null hypothesis is true. This comparison allows one to compute the probability that the observed outcome could have been due to chance alone.

Hypothesis: A statement of (or conjecture about) the relationships among the variables that a researcher intends to study. Hypotheses are sometimes testable statements of relations. In such cases, they are usually thought of as predictions which, if confirmed, will support a theory.

Independent Variable: The presumed cause in a study. Also a variable that can be used to predict the values of another variable. 
Interaction Effect: The joint effect of two or more independent variables on a dependent variable. Interaction effects occur when independent variables not only have separate effects but also have combined effects on a dependent variable. Interaction effects occur when the relation between two variables differs depending on the value of another variable.

Intervention: A public health initiative designed to control a disease or event outcome by testing hypotheses and evaluating its effectiveness and impact on the health status of a target population.

Level of Significance: The level of statistical significance is the probability that a result would be produced by chance alone. The level of significance indicates the risk or probability of committing an error (Type I Error in hypothesis testing). The level of significance is stated as a probability, often abbreviated $p$, followed by a number, for example, $p<.01$. The smaller the number, the smaller the chance of a Type I Error and the more statistically significant the finding.

Likert Scale: A type of composite measure developed by Rensis Likert in an attempt to improve the levels of measurement in social research through the use of standardized response categories in survey questionnaires. Likert items are those using such response categories as strongly agree, agree, disagree, and strongly disagree. Such items may be used in the construction of true Likert scales and may also be used in the construction of other types of composite measures.

Linear Relation (or Correlation): A relationship that, when plotted on a graph, forms a straight line. It forms a straight line because the direction and the rate of change in one variable are constant with respect to changes in the other.

Logistic Regression Analysis: A kind of regression analysis used when the dependent variable is dichotomous and scored 0,1. It is usually used for predicting whether something will happen or not, such as graduation, business failure, heart disease-anything that can be expressed as event/nonevent.
Longitudinal Study: Consists of at least two sets of observations or ongoing surveillance of the study population over a given followup period. Longitudinal studies may involve either a fixed cohort or a dynamic population. A fixed cohort is a group of subjects identified at a hypothetical point in time and followed for a given period for detection of new cases of disease (or event). A dynamic population may gain and lose subjects over the course of the followup period.

Matching: In connection with experiments, the procedure whereby pairs of subjects are matched on the basis of their similarities on one or more variables, and one member of the pair is assigned to the experimental group and the other to the control group.

McNemar Test: A test for paired proportions that analyzes the number of disagreements when the measured characteristic is nominal.

Mean: A number that in some sense represents the central value of a set of numbers. Also, the average. To [calculate] the mean, you add up the values for each case and divide the total by the number of cases.

Mean Score: An arithmetic average of all measurement scores.

Median: The middle score in a set of ranked scores. When the number of scores is even, there is no single middle score; in that case, the median is found by taking an average of the two middle scores.

Metaphorically: Speaking figuratively in which a term is transferred from the object it ordinarily designates to an object it may designate only by implicit comparison or analogy.

Minority Health Communication Model: Consists of five phases of communication development: consultation with "experts" who are knowledgeable of the target population, consultation with community representatives, setting goals, developing communication strategies, and evaluation

Mode: The most common (most frequent) score in a set of scores.

Morbidity: The incidence or prevalence of a disease or all diseases in a population. 
n (Lower case): Number in a sample, as opposed to in a population, or number of cases in a subgroup.

N (Upper case): Number of subjects or cases in a particular study, number of individuals in a population, or number of variables in a study.

Nonparametric Statistics: Statistical techniques designed to be used when the data heing analyzed depart from the distributions that can be analyzed with parametric statistics. The chisquare test is probably the best known example.

Normal Distribution: A purely theoretical continuous probability distribution in which the horizontal axis represents all possible values of a variable and the vertical axis represents the probability of those values occurring. The scores on the variable (often expressed as zscores) are clustered around the mean in a symmetrical, unimodal pattern known as the hell-shaped curve or normal curve. In a normal distribution, the mean, median, and mode are all the same. There are many different normal distributions, one for every possible combination of mean and standard deviation.

Null Hypothesis $\left(\mathbf{H}_{\mathbf{o}}\right)$ : The hypothesis that two or more variables are not related or that two or more statistics (e.g., means for two different groups) are not the same. In accumulating evidence that the null hypothesis is false, the researcher indirectly demonstrates that the variables are related or that the statistics are different. The null hypothesis is the core idea in hypothesis testing.

Odds Ratio: A ratio of one odds to another. The odds ratio is a measure of association but, unlike other measures of association, "1.0" means that there is no relationship between the variables. The size of any relationship is measured by the difference (in either direction) from 1.0. An odds ratio less than 1.0 indicates an inverse or negative relation; an odds ratio greater than 1.0 indicates a direct or positive relation.

One-Way ANOVA: Analysis of variance with only one independent variable.

Outcome Variable: Another term for dependent variable, used mainly in nonexperimental research to refer to the presumed effect.

$\boldsymbol{p}$ Value: Usually found in an expression such as $p<$ 05. This expression means: "The probability $(p)$ that this result could have been produced by chance is less than $(<)$ five percent (.05)." Thus, the smaller the number, the greater the likelihood that the result expressed was not merely due to chance. For example, $p<.001$ means that the odds are a thousand to one cone-tenth of $1 \%$ ) against the result being a fluke. What is being reported $(.05, .001$, and so on) is an alpha level or significant level. The $p$ value is the actual probability associated with an obtained statistical result; this is then compared with the alpha level to see whether that value is (statistically) significant.

Pap Test (or Smear) or Papanicolaou's Test: An exfoliative cytologic staining procedure for the detection and diagnosis of various conditions, particularly malignant and premalignant conditions of the female genital tract (cancer of the vagina, cervix, and endometrium), in which cells which have been desquamated from the genital epithelium are obtained by smcars, fixed and stained, and examined under the microscope for evidence of pathologic changes.

Parameter: The most common use of a parameter is for a characteristic of a population, or of a distribution of scores, described by a statistic such as a mean or a standard deviation.

Parametric Statistics: Statistical techniques designed for use when data have certain characteristics-usually when they approximate a normal distribution and are measurable with interval or ratio scales. Also, statistics used to test hypotheses about population parameters.

Paucity of Data: Little cmpirical information exists.

Policy Approach: A focus on changing or improv ing an organization's policy rather than directing educational programs at individuals within the organization.

Population: A group of persons (or institutions, events, or other subjects of study) that one wishes to describe or about which one wishes to generalize. To gencralize about a population, one often studies a sample that is meant to be representative of the population. 
Post Hoc Comparison: $\Lambda$ test of the statistical significance of differences between group means calculated after (post) having done an ANOVA or regression analysis that shows an overall difference.

Posttest: $A$ test given or measurement taken after an experimental treatment.

Posttest Data: Information collected after intervention.

Power of the Test: Broadly, the ability of a technique, such as a statistical test, to detect relationships; specifically, the probability of rejecting a null hypothesis when it is false-and therefore should be rejected. The power of a test is calculated by subtracting the probability of a Type II Error from 1.0. The maximum total power a test can have is 1.0 ; the minimum is zero. Also called statistical power.

PRECEDE (Predisposing, Reinforcing, and Enabling Causes in Educational/Environmental Diagnosis and Evaluation) Model: A diagnostic (or needs assessment) phase of a health promotion intervention. This model has three categories of factors affecting individual or collective hehavior, including organizational actions in relation to the environment, each of which has a different type of influence on behavior: Predisposing factors include a person's or population's knowledge, attitudes, beliefs, values, and perceptions that facilitate or hinder motivation for change; reinforcing factors, the rewards received and the feedback the learner receives from the others following adoption of the behavior may encourage or discourage continuation of the behavior; enabling factors are those skills, resources, or barriers that can help or hinder the desired behavioral changes as well as environmental changes. The skills required for a clesired behavior to occur also qualify as enabling factors. Enabling factors thus include all the factors that make possible a desired change in behavior or in the environment. Any given behavior can be explained as a function of the collective influence of these three types of factors.

Pretest: A test given or measurement taken before an experimental treatment begins. By contrast- ing the results of the pretest with those of the posttest, researchers gain evidence about the effects of the treatment. $\Lambda$ pretest is also a trial run used to assess some part of an instrument or procedure.

Pretest Data: Information collected prior to intervention.

Pretest-Posttest Control Group Design: The classical experimental design in which subjects are assigned randomly to an experimental group that is exposed to a stimulus and to a control group that is not exposed to the stimulus. Each group is tested on the dependent variable before and after the experimental group is exposed to the stimulus.

Prevalence: The number of cases of a disease (or event) that are present in a population at one point in time. Prevalence cases are existing cases for which the duration of the disease may remain unknown to the investigator.

Prevalence Rate: The number of people in a population who have a disease (or event) at a given time; the numerator of the rate is the number of existing cases of disease at a point in time and the denominator is the total population.

Prevention: Taking the necessary precautions to prevent the occurrence of cliseases.

Primary Prevention of Cancer: The complete avoidance of exposure to a carcinogen.

Probability: The likelihood that a particular event or relationship will occur.

Process Data: A series of operations, events, or steps leading to the achievement of a specific result.

Prochaska's Scale or Stages of Change Model: The model poses behavioral change as a cyclical phenomenon with distinct stages, including precontemplation, contemplation, action, and maintenance. People who smoke cycle forward and back through the different stages. Relapse represents the termination of the action or maintenance phase. Precontemplation is a stage in which people are not seriously thinking about changing, at least not in the next 6 months. A 6 month criterion is used because that seems to be about as far ahead in time that people seri- 
ously plan health behavior changes. Contemplation is the period during which people are seriously thinking about changing an unhealthy behavior in the next 6 months. Action is the 6-month period following an overt modification of an unhealthy behavior. Maintenance is the period from 6 months after an overt behavior change until the problem behavior is finally terminated. Termination is defined as zero temptation across all problem situations and maximum confidence in one's ability to resist relapse across all problem situations. Maintenance is a period of continued change, whereas termination is a much more stable state.

Qualitative Analysis: The nonnumerical examination and interpretation of observations for the purpose of discovering underlying meanings and patterns of relationships. This is most typical of field research and historical research.

Qualitative Data: Nonnumerical research data.

Quantitative Methods: Research methods that emphasize precise, objective, and generalizable findings.

Questionnaire: A group of written questions to which subjects respond. Some restrict the use of the term "questionnaire" to written responses.

Randomization: A technique for assigning experimental subjects to experimental and control groups randomly.

Random Sampling: The selection of subjects for a study in such a way as to give each potential subject in the target population a known probability of being included in the study. The case in which all selection probabilities are equal is known as simple random sampling-i.e., in this design, each person has the same probability of being sampled. The cornerstone of survey research, random sampling ensures that the study population is representative (on the average) of the target population with respect to all factors relevant to the investigator, including the joint distribution of the disease (or event) and its determinants. Random sampling, therefore, allows the researcher to make statistical inferences regarding certain target population parameters from his or her estimates in the study pop- ulation. Thus, the researcher may conclude, with a certain degree of confidence, that his or her findings are not due to errors of sampling.

Random Variable: A variable that varies in ways the researcher does not control; a variable whose values are randomly determined. "Random" refers to the way the events, values, or subjects are chosen or occur, not to the variable itself. Men and women are not random, but gender could be a random variable in a research study; the gender of subjects included in the study could be left to chance and not controlled by the researcher.

Response Rate: The number of persons participating in a survey, divided by the number selected in the sample, in the form of a percentage. This is also called the completion rate or, in selfadministered surveys, the return rate, i.e., the percentage of questionnaires sent out that are returned.

Risk Factor: A variable that is believed to be related to the probability of an individual developing a disease.

Robust: In statistics, a somewhat imprecise term that is applied to a procedure that is relatively insensitive to violations of the assumptions on which it is based.

Sample: A group of subjects selected from a larger group in the hope that studying this smaller group (the sample) will reveal important [information] about the larger group (the population).

Sampling Frame: A list or other record of the population from which the sampling units are drawn.

Scale: A type of composite measure composed of several items that have a logical or empirical structure among them. Examples of scales include Bogardus social distance, Likert, and Thurstone scales.

Scheffé's Procedure: A test of statistical significance used for post hoc multiple comparisons after a regression analysis or an ANOVA. Among its main features are that it is a conservative test (it tends to err on the side of underestimating significance) and that it deals well with unequal cell sizes. 
SEER: One of the sources of cancer data for this monograph is the Surveillance, Epidemiology, and End Results (SEER) Program of NCI. As a continuing project of $\mathrm{NCI}$, the SEER Program is responsible for monitoring the impact of cancer in the general U.S. population. Information from 11 SEER geographic areas and from the Alaska Area Native Health Service are used in this monograph. These areas are the States of Connecticut, Hawaii, Iowa, New Mexico, and Utah and the metropolitan areas of Atlanta (including 10 rural counties), Detroit, Los Angeles, San Francisco/Oakland, San Jose/Monterey, and Seattle/Puget Sound. These areas cover about 14 percent of the total U.S. population. The Alaska Area Native Health Service also receives support from $\mathrm{NCI}$ and provides cancer incidence data for its Alaska Native population that are compatible with the data from the SEER areas. Participants in the SEER Program were selected for their ability to operate and maintain a population-based cancer reporting system and for the variety and size of population subgroups within their areas (e.g., racial/ethnic, urban and rural) which are of special epidemiologic interest.

Self-Efficacy: A concept from the Social Learning Theory that implies a mental or cognitive state of taking control. It is the belief in the ability to behave in ways necessary to produce identified outcomes. It is also a perception of one's own capacity for success in organizing and implementing a pattern of behavior that is new, based largely on experience with similar actions or circumstances encountered or observed in the past. In addition to its influence on behavior, self-efficacy affects thought patterns and emotional reactions that may alleviate anxiety and enhance coping ability.

Significance: The degree to which a research finding is meaningful or important.

Significance Testing: Using statistical tests (such as chi square, $t$ test, or $F$ test) to determine how likely it is that observed characteristics of samples have occurred by chance alone in the populations from which the samples were selected. If the observed characteristics in the samples are unlikely to be due to chance alone, the charac- teristics are deemed statistically significant.

Smoking Cessation: To cease or to stop smoking.

Smoking Rate: The number of people in a specific population who smoke at a given time. The numerator of the rate is the number of people who smoke at a point in time, and the denominator is the total number of people in that specific population.

Social Learning Theory (SLT): Inherent in the social learning conception is the idea that people self-regulate their environments and actions. Although people are acted upon by their environments, they also help create their surroundings. According to this model, learning takes place through three processes: (1) direct experience, (2) indirect or vicarious experience from observing others (modeling), and (3) the storing and processing of complex information in cognitive operations that enable one to anticipate the consequences of actions, represent goals in thought, and weigh evidence from various sources to assess one's own capabilities.

Solomon Four-Group Design: A very sophisticated experimental design that takes into account factors associated with external as well as internal validity. The design is set up to determine several factors at once: assess the effect of the treatment in relation to the control group, determine the effect of the pretest, and explain the interaction between the treatment conditions and the pretest. Random assignment of subjects to groups and inclusion of groups that are not pretested add to the significance of results of the experiment. This results from combinations of the posttest only and pretest-posttest. The design actually has two experiments going on at once and thus provides replications of the study. Internal Validity can be defined as control for all influences between the groups being compared in an experiment, except for the experimental group. External Validity is concerned with the researcher being able to generalize the findings of an experiment. In an attempt to control threats to internal validity in the behavioral sciences, the investigator runs the risk of creating an unreal situation from which generalization to other settings is impossible. 
Standard Deviation (SD): A statistic that shows the spread or dispersion of scores in a distribution of scores; in other words, a measure of dispersion. The more widely the scores are spread out, the larger the standard deviation.

Standardized Mortality Ratios: A type of age adjustment. It is the number of observed deaths divided by the number of expected deaths.

Statistic: A number that describes some characteristic of a variable or of a group of data-such as a mean or a correlation coefficient.

Statistically Correlated: The degree to which a linear predictive relationship exists between random variables, as measured by a correlation coefficient (qv).

Statistics: Numerical summaries of data obtained by measurement and computation.

Student's $\boldsymbol{t}$ Distribution: A family of theoretical probability distributions used in hypothesis testing. As with normal distributions, $t$ distributions are unimodal, symmetrical, and bell shaped. The $t$ distribution is especially important for interpreting data gathered on small samples when the population variance is unknown. The larger the sample, the more closely the $t$ approximates the normal distribution. For samples greater than 120 , they are practically equivalent.

Study: A research project.

Study Population: Group of subjects for which observations or data are made.

Survey: A research design in which a sample of subjects is drawn from a population and studied (usually interviewed) to make inferences about the population.

Target Population: The larger group to which researchers would like to make inferences.

Theory: A statement or group of statements about how some part of the world works-frequently explaining relations among phenomena.

Type I Error: An error made by wrongly rejecting a true null hypothesis. This might involve incorrectly concluding that two variables are related when they are not or wrongly deciding that a sample statistic exceeds the value that would be expected by chance. Also called alpha error.
Type II Error: An error made by wrongly accepting (or retaining or failing to reject) a false null hypothesis. Also called beta error.

Unimodal Distribution: A distribution with only one mode.

Variable: Any finding (an attribute or characteristic) that can change, that can vary, or that can be expressed as more than one value or in various values or categories.

Variance: A measure of the spread of scores in a distribution of scores; that is, a measure of dispersion. The larger the variance, the further the individual cases are from the mean. The smaller the variance, the closer the individual scores are to the mean.

Wilcoxon Signed Ranks Test: The most commonly used nonparametric test for the difference between two paired samples. It tests the hypothesis that the medians, rather than the means, are equal in the two paired samples. It is an excellent alternative to the $t$ test because it is almost as powerful (rejecting the null hypothesis when it is false) as the $t$ test in detecting an actual difference when the observations follow a normal distribution. When the observations are not normally distributed, it is more powerful than the $t$ test.

z-Score: The most commonly used standard score. In $z$-score notation, the mean is 0 and a standard deviation is 1 . Thus a $z$-score of 1.25 is one and one-quarter standard deviations above the mean.

\section{REFERENCES FOR DEFINITIONS}

Byar DP, Freedman LS. The importance and nature of cancer prevention trials. Semin Oncol 1990; 17:413-24.

Dawson-Saunders B, Trapp RG. Basic and clinical biostatistics. Norwalk, CT: Appleton \& Lange, 1990.

Dorland, WA. Dorland's illustrated medical dictionary. 27th ed. Philadelphia, PA: W.B. Saunders Company, 1988. 
Elder JP. Motivating health behavior. Albany, NY: Delmar Publishers Inc., 1994:109, 114.

Goldberg DN, Hoffman AM, Farinha MF, Marder DC, Tinson-Mitchem L, Burton D, et al. Physician delivery of smoking cessation advice based on the Stages-of-Change Model. Am J Prev Med 1994; 10:267-74.

Green LW, Kreuter MW. Health promotion planning: an educational and environmental approach. $2 \mathrm{~d}$ ed. Mountainview, CA: Mayfield Publishing Company, 1991:1, 28, 29, 156, 157, 159, 160.

Greenwald P, Kramer BS, Weed DL, editors. Cancer prevention and control. New York: Marcel Dekker, Inc., 1995.

Hansen MH, Hurwitz WN, Madow WG. Sample survey methods and theory. Vol 2: Theory. New York: Wiley, 1953

Kleinbaum DG, Kupper LL, Morgenstern H. Epidemiologic research: principles and quantitative methods. Belmont, CA: Lifetime Learning Publications, 1982.

MacMahon B, Pugh TF. Epidemiology: principles and methods. Boston: Little, Brown, 1970.

Miller BA, Kolonel LN, editors. Racial/ethnic patterns of cancer in the United States, 1988-1992 (SEER Monograph). NIH Publication No. 96-4104. Bethesda, MD: National Cancer Institute, 1996.
Neutens JJ, Rubinson L. Research techniques for the health sciences. Needham Heights, MA: Allyn \& Bacon, 1997:71, 74, 80

Prochaska JO. Assessing how people change. Cancer 1991;67:805-7.

Schunk DH, Carbonari JP. Self-efficacy models. In: Matarazzo JD, Weiss SM, Herd JA, et al., editors. Behavioral health: a handbook of health enhancement and disease prevention. New York: Wiley, 1984:230-47.

Spiegel MR. Schaum's outline of theory and problems of probability and statistics. New York: McGraw-Hill, 1975

Vogt WP. Dictionary of statistics and methodology: a nontechnical guide for the social sciences. Newbury Park, CA: Sage Publications, Inc., 1993:4, 7, 34, 42, $47-9,64,67,82,87,94,108,110,113,128,129,131$, $137,138,141,149,155,158,159,161,163-5,175-7$, $184,188,200,202,204,210,217,219,221,224,225$, 228,241 . Reprinted by permission of publisher, Sage Publications, Inc.

Webster's II new Riverside university dictionary. Boston: Riverside Publishing Company, 1984. 


\section{Appendix $B$ \\ Bibliographies Associated With Studies}

\section{Prevention of Cervical Cancer in Alaska Native Women \\ James Berner, M.D., and Anne P. Lanier, M.D., M.P.H., Principal Investigators}

Stillwater B, Echavartia VA, Lanier AP. Pilot test of a cervical cancer prevention video developed for Alaska Native women. Public Health Rep 1995; 110:211-4.

\section{North Carolina Native American Cervical Cancer Prevention Project}

Mark B. Dignan, Ph.D., M.P.H., Principal Investigator

Dignan M, Michielutte R, Blinson K, Sharp P, Wells $\mathrm{HB}$, Sands E. Cervical cancer prevention: an individualized approach. Alaska Med 1993;35:279-84.

Michielutte R, Sharp $\mathrm{P}$, Dignan MB, Blinson $\mathrm{K}$. Cultural issues in the development of cancer control programs for American Indian populations. I Health Care Poor Underserved 1994;5:280-96.

Spangler JG, Dignan MB, Michielutte R. Smokeless tobacco use among American Indian women-Southeastern North Carolina, 1991. MMWR 1995; 44:113-7.

Bell RA, Shaw HA, Dignan MB. Dietary intake of Lumbee Indian women in Robeson County, North Carolina. J Am Diet Assn 1995;95:1426-8.

Blinson K, Dignan M, Michielutte R. The cost of conducting face-to-face household interviews in a rural, Native American population: the North Carolina Native American Cervical Cancer Prevention Project. Cancer 1996;78(7 Suppl):1587-91.

Dignan M, Michielutte R, Blinson $\mathrm{K}$, Wells HB, Case LD, Sharp $P$, et al. Effectiveness of health education to increase screening for cervical cancer among eastern-band Cherokee Indian women in North Carolina. I Nat] Cancer Inst 1996;88:1670-6.
Spangler J, Dignan M, Michielutte R. Correlates of tobacco use among Native American women in western North Carolina. Am J Public Health 1997;87:108-11.

Spangler JG, Bell RA, Dignan MB, Michielutte R. Prevalence and predictors of tobacco use among Lumbee Indian women in Robeson County, North Carolina. J Community Health 1997;22:115-25.

The Wai anae Coast Cancer Research Project Richard Banner, M.D., M.P.H.,

Principal Investigator

Matsunaga DS, Enos R, Gotay CC, Banner RO, DeCambra A, Hammond OW, et al. Participatory research in a Native Hawaiian community. The Wai anae Cancer Research Project. Cancer 1996;78(7 Suppl):1582-6.

Banner RO, DeCambra $\mathrm{H}$, Enos R, Gotay C, Hammond OW, Hedlund N, et al. A breast and cervical cancer project in a Native Hawaiian community: Wai anae Cancer Research Project. Prev Med $1995 ; 24: 447-53$.

Matsunaga DS, Enos R, Gotay CC, Banner RO, DeCambra $\mathrm{H}$, Hammond OW, et al. Participatory research in a Native Hawaiian community: the Wai anae Cancer Research Project. Cancer 1996; 78:1582-6.

\section{Tobacco Policy Intervention in Northwest Indian Tribes}

Edward Lichtenstein, Ph.D.

Principal Investigator

Glasgow RE, Lichtenstein E, Hall R, Meyers GB, Wilder D, Gilbert-McRae S. Indoor smoking policies of Indian tribes in the northwestern United States. Tob Control 1993;2:35-7. 
Glasgow RE, Lichtenstein E, Wilder D, Hall R, GilbertMcRae S, Liberty B. The Tribal Tobacco Policy Project: working with Northwest Indian tribes on smoking policies. Prev Med 1995;24:434-40.

Glasgow RE, Boles SM, Lichtenstein E, Strycker LA. The tobacco policy rating form: a tool for evaluating worksite and tribal smoking control policies. Tob Control 1996;5:286-91.

Hall RL, Lichtenstein E, Burhansstipanov L, Davis SM, Hodge F, Schinke S, et al. Tobacco use policies and practices in diverse Indian settings. Am Indian Culture Res J 1995;19:165-80.

Hall RL, Viles CA, Lichtenstein E, Glasgow RE, Lopez KL. Rapid assessment procedures to describe tobacco practices at sites managed by Indian tribes. Tob Control 1995;4:156-61.

Lichtenstein E, Glasgow RE, Lopez L, Hall R, GilbertMcRae S, Meyers GB. Promoting tobacco control policies in Northwest Indian tribes. Am J Public Health 1995;85:991-4.

Lichtenstein E, Lopez K, Glasgow RE, Gilbert-McRae $S$, Hall RL. Effectiveness of a consultative intervention to promote tobacco control policies in Northwest Indian tribes: integrating experimental evaluation and service delivery. Am J Community Psychol 1996;24:639-55.

\section{Smoking Cessation for American Indians \\ Felicia Schancbe Hodge, Dr.P.H., \\ Principal Investigator}

Hodge F, Kipnis P. Demoralization: a useful concept for case management with American Indians. In: Manoleas $P$, editor. The cross-cultural practice of clinical case management in mental health. New York: The Haworth Press, 1996:79-95.

Iodge F, Fredericks L, Kipnis P. Patient and smoking patterns in northern California American Indian clinics: urban rural contrasts. Cancer 1996; 78 (Suppl):1623-8.

Hodge F, Cummings S, Fredericks L, Kipnis P, Williams $M$, Teehee $K$. Prevalence of smoking among adult American Indian clinic users in northern California. Prev Med 1995;24:441-6.
Hodge FS. Tobacco control leadership in American Indian communities. In: Slama $\mathrm{K}$, editor. Tobacco and health. New York: Plenum Press, 1995:375-8.

Hall R, Lichtenstein E, Burhansstipanov L, Davis S, Hodge F, Schinke $S$, et al. Tobacco use policies and practices in diverse Indian settings. Am Indian Culture Res J 1995;19:165-80.

Robinson R, Shelton D, Hodge F, Lew R, Lopez E, Toy $P$, et al. Tobacco control capacity index for communities of color in the United States. In: Slama $\mathrm{K}$, editor. Tobacco and health. New York: Plenum Press, 1995:359-65.

\section{Southwestern Cancer Prevention Project for American Indians}

Sally M. Davis, Ph.J., Principal Investigator

Cunningham-Sabo I, Davis SM. Pathways to Health: a health promotion and cancer prevention project for American Indian youth. Alaska Med 1993;35:2758, 296.

Cunningham-Sabo LD, Davis SM, Kochler KM, Fugate ML, DiTucci JA, Skipper BJ. Food preferences, practices, and cancer-related food and nutrition knowledge of southwestern American Indian youth. Cancer 1996,78(7 Suppl):1617-22.

Davis SM, Lambert LC, Cunningham-Sabo L, Skipper BJ. Tobacco use: baseline results from Pathways to Health, a school-based project for southwestern American Indian youth. Prev Med 1995;24:454-60.

Hall R, Lichtenstein E, Burhansstipanov L. Davis SM, Hodge F, Schinke S, et al. Tobacco use policies and practices in diverse Indian settings. Am Indian Culture Res J 1995;19:165-80.

Osborn KL, Davis SM, Slattery M, Giuliano A, Teufel $\mathrm{N}$, Joe J, et al. Four Corners Research Consortium for Native Americans and cancer research. Cancer 1996;78(7 Suppl):1629-32.

\section{Reducing Cancer Risks Among Native American Adolescents}

Steven Schinke, Ph.D., Principal Investigator

Schinke SP, Singer B, Cole K, Contento IR. Reducing cancer risk among Native American adolescents. Prev Med 1996;25:146-55. 
Hall R, Lichtenstein E, Burhansstipanov L, Davis SM, Hodge F, Schinke $S$, et al. Tobacco use policies and practices in diverse Indian settings. Am Indian Culture Res J 1995;19:165-80.

Schinke SP. Behavioral approaches to illness prevention for Native Americans. In: Kato PM, Mann T, editors. Handbook of diversity issues in health psychology. New York: Plenum Press, 1996:367-87.

Schinke SP, Cole KC. Methodological issues in conducting alcohol abuse prevention research in ethnic communities. In: Langton PA, Epstein LG, Orlandi
MA, editors. Challenge of participatory research: preventing alcohol-related problems in ethnic communities. CSAP Cultural Competence Series 3. Rockville, MD: Substance Abuse and Mental Health Services Administration, Center for Substance Abuse Prevention, 1995:129-47.

Schinke SP, Cole KC. Prevention in community settings. In: Botvin GJ, Schinke SP, Orlandi MA, editors. Drug prevention with multiethnic youth. Thousand Oaks, CA: Sage Publications, 1995:215-32. 


\section{Appendix $C$ \\ Highlights of Avoidable Mortality From Cancer in Native American Populations Program}

\begin{tabular}{|c|c|c|c|}
\hline & Site 1 & Site 2 & Site 3 \\
\hline Project title: & $\begin{array}{l}\text { Prevention of Cervical } \\
\text { Cancer in Alaska Native } \\
\text { Women }\end{array}$ & $\begin{array}{l}\text { North Carolina Native } \\
\text { American Cervical } \\
\text { Cancer Prevention } \\
\text { Project }\end{array}$ & $\begin{array}{l}\text { The Wai anae Coast } \\
\text { Cancer Research Project }\end{array}$ \\
\hline Name of organization: & $\begin{array}{l}\text { Alaska Area Native } \\
\text { Health Services }\end{array}$ & $\begin{array}{l}\text { Wake Forest University } \\
\text { School of Medicine }\end{array}$ & $\begin{array}{l}\text { The Wai anae Coast } \\
\text { Comprehensive Health } \\
\text { Center }\end{array}$ \\
\hline Location of site: & Anchorage, AK & Winston-Salem, NC & Waìanae, $\mathrm{HI}$ \\
\hline $\begin{array}{l}\text { Type of cancer } \\
\text { screening targeted: }\end{array}$ & Cervical & Cervical & Breast and cervical \\
\hline Group studied: & Alaska Native women & American Indian women & Hawaiian women \\
\hline Age of study group: & 20 and older & 18 and older & 18 and older \\
\hline Control group: & Yes & Yes & No \\
\hline Number in study: & 481 urban women & $\begin{array}{l}\text { 1,020 Cherokee women } \\
983 \text { Lumbee women }\end{array}$ & 500 \\
\hline $\begin{array}{l}\text { Primary objective of } \\
\text { project: }\end{array}$ & $\begin{array}{l}\text { Promote knowledge and } \\
\text { awareness of cervical } \\
\text { cancer, its risk factors, } \\
\text { and screening programs. } \\
\text { Determine the number } \\
\text { of women receiving } \\
\text { Pap test. }\end{array}$ & $\begin{array}{l}\text { Increase proportion of } \\
\text { women } 18 \text { and older } \\
\text { who receive Pap smears } \\
\text { at appropriate intervals } \\
\text { and return for followup } \\
\text { care when necessary. }\end{array}$ & $\begin{array}{l}\text { Test cffectiveness of a } \\
\text { culturally sensitive, com- } \\
\text { munity-driven interven- } \\
\text { tion as a means of } \\
\text { incrcasing breast and } \\
\text { cervical cancer screen- } \\
\text { ing practices, as well as } \\
\text { increase KAB scores } \\
\text { among Native Hawaiian } \\
\text { women. }\end{array}$ \\
\hline
\end{tabular}




\section{Highlights of Avoidable Mortality From Cancer in Native American Populations Program (continued)}

Site 1

Study design:

Comparison of interven-

tion and control groups

at baseline and later:

Comparison of same intervention group at

baseline and later:
Site 2

Site 3
Provision of clinic serv- Use of tribal rolls to ices and education about cervical cancer and screening

Yes

Yes

Yes

Yes select individuals ran-

domized to four groups
Formation of healthactivated mutual support groups (kokua groups)

Type of intervention: Clinic Tribal Community

\section{Data collection method:}

Face-to-face interviews and surveys; medical record review
Household interviews

Self-administered questionnaire

Percent improvement
in screening rates
attributable to inter-
vention:

Percent improvement in screening rates vention:
Alaska Native women who had one or more Pap tests increased from 41 percent to 49 percent pre- and postintervention, respectively. There was a significant increase in women's knowledge of cervical cancer, risk factors, and screening.
Approximately 73 percent of all Cherokee women who received the intervention reported having a Pap test in the past year, compared to 64 percent of women who did not receive the intervention (adjusted odds ratio $[\mathrm{OR}]=2.06$, 95\% confidence interval $[\mathrm{CI}]=1.14-3.72$ ). Among the Lumbee women, there was no significant intervention effect on the number of women who reported having received a Pap test in the past year.
With respect to having a Pap test, 29 percent of the women reported that they were planning to make an appointment (compared to 19 percent pre-kokua group) and 64 percent said they were in adherence (compared to 59 percent pre-kokua group). This contrast was significant $(z=-5.45, p<.001)$ by Wilcoxon signed rank test. 


\section{Highlights of Primary Prevention of Cancer in Native American Populations Program}

\begin{tabular}{lllll} 
& \multicolumn{1}{c}{ Site 4 } & \multicolumn{2}{c}{ Site 5 } & \multicolumn{1}{c}{ Site 6 7} \\
\hline Project title: & Tobacco Policy & Smoking Cessation & Southwestern & Reducing Cancer \\
& Intervention in & for American & Cancer Prevention & Risks Among \\
& Northwest Indian & Indians & Project for & Native American \\
& Tribes & & American Indians & Adolescents
\end{tabular}

\begin{tabular}{lllll}
\hline Name of & Oregon Research & Center for & University of & Columbia \\
organization: & Institute & American Indian & New Mexico & University \\
& & Research and & \\
& Education & &
\end{tabular}

Location of site: $\quad$ Eugene, OR $\quad$ Berkeley, CA Albuquerque, NM New York, NY

\begin{tabular}{|c|c|c|c|c|}
\hline $\begin{array}{l}\text { Target health } \\
\text { behavior: }\end{array}$ & Tobacco use policy & Smoking cessation & $\begin{array}{l}\text { Avoidance of } \\
\text { smoking and } \\
\text { smokeless tobacco; } \\
\text { low-fat, high-fiber } \\
\text { diet }\end{array}$ & $\begin{array}{l}\text { Tobacco use } \\
\text { and dietary } \\
\text { modification }\end{array}$ \\
\hline
\end{tabular}

\begin{tabular}{lllll}
\hline Group studied: & 39 tribes in & American Indian & Navajo and Pueblo Native American \\
& Northwest & clinic users in & Southwest Indian adolescents \\
& northern California & tribes
\end{tabular}

\begin{tabular}{|c|c|c|c|c|}
\hline $\begin{array}{l}\text { Age of study } \\
\text { group: }\end{array}$ & $\begin{array}{l}\text { Tribe unit of } \\
\text { analysis }\end{array}$ & 18 and older & $\begin{array}{l}\text { 5th- and } 7 \text { th-grade } \\
\text { students and their } \\
\text { families }\end{array}$ & 8 to 13 years \\
\hline
\end{tabular}

\begin{tabular}{llll}
\hline Number in study: & 39 tribes & 1,369 & 1,589 \\
& & 135 \\
\hline $\begin{array}{l}\text { Intervention } \\
\text { group: }\end{array}$ & $\begin{array}{l}19 \text { tribes in early } \\
\text { intervention, } 20 \text { in } \\
\text { late intervention }\end{array}$ & 695 & 103
\end{tabular}


Highlights of Primary Prevention of Cancer in

Native American Populations Program (continued)

Site 4

Site 5

Site 6

Site 7

Control group:

669

31

Primary objective Develop a consultaof project:

tive process to facili- tate tribes in creating

and implementing

more explicit and

stringent tobacco

use policies; assess

impact of policy

intervention and

evaluate relationship

of tobacco use poli-

cies and antitobacco

behavior; develop a

manual and guide-

lines for tobacco

policy development

and implementation

for use by tribal

councils and organi-

zations
Increase long-term

smoking cessation

among American

Indian populations

through a repro-

ducible clinic-

based program
Develop, implement, and evaluate a program to promote avoidance of smoking and use of smokeless tobacco and promote a low-fat, high-fiber diet
Reduce cancer risks among Native American youth by developing skills to resist pressures to use tobacco or eat poorly

$\begin{array}{llll}\text { No } & \text { Yes } & \text { Yes } & \text { Yes }\end{array}$

\section{Study design:}

Randomization of tribes to immediate (early) or delayed (late) intervention following baseline assessment; continuous and dichotomous summary measures
Randomized clinical trial; matched pairs

Pathways to Health cancer prevention curriculum used
17 initial intervention sessions with semiannual booster sessions; four cells: (1) tobacco use prevention and dietary modification, (2) tobaccu use prevention, (3) dietary modification, (4) no intervention. Urban and reservation sites and semiannually 


\section{Highlights of Primary Prevention of Cancer in Native American Populations Program (continued)}

Site 4

Site 5

Site 6

Site 7

\begin{tabular}{|c|}
\hline $\begin{array}{l}\text { Comparison } \\
\text { of same } \\
\text { intervention } \\
\text { group at baseline }\end{array}$ \\
\hline
\end{tabular}

\begin{tabular}{|c|c|c|c|}
\hline $\begin{array}{l}\text { Type of } \\
\text { intervention: }\end{array}$ & $\begin{array}{l}\text { Tribal policy } \\
\text { change }\end{array}$ & Clinic & School and family \\
\hline
\end{tabular}

Data collection method:
Phone interview

data on tobacco

policies from 39

tribes at baseline

and followup
Self-administered questionnaire at baseline; 6- and 18 month followup personal interviews in homes; process interviews
Questionnaires and body composition measures administered before and after curriculum
Pre- and posttest questionnaires; eating habit questionnaires; cotinine tests

\section{Intervention} outcomes:

\begin{tabular}{|c|c|}
\hline $\begin{array}{l}\text { Significant tobacco } \\
\text { policy changes for } \\
\text { both the tribes that } \\
\text { received consulting } \\
\text { help first and those } \\
\text { that received con- } \\
\text { sulting help later. } \\
\text { Policies were } \\
\text { stronger, and more } \\
\text { tribes were smoke- } \\
\text { free in all three } \\
\text { common areas by } \\
\text { the end of the pro- } \\
\text { gram. The number } \\
\text { of tribes having } \\
\text { smoke-free policies } \\
\text { in all three common } \\
\text { areas more than } \\
\text { doubled in both } \\
\text { groups of tribes. }\end{array}$ & $\begin{array}{l}\text { Mean quit rate in } \\
6 \text {-month followup } \\
\text { for intervention } \\
\text { group was } .1272 \\
\text { compared to mean } \\
\text { control quit rate of } \\
.064 . \text { The differ- } \\
\text { ence between } \\
\text { these two was sig- } \\
\text { nificant ( }<<.01 \text { ). } \\
\text { The intervention } \\
\text { had a greater effect } \\
\text { on light smokers } \\
\text { than on heavier } \\
\text { smokers. }\end{array}$ \\
\hline
\end{tabular}

Baseline nutrition

questionnaire

shows students

lack essential

knowledge about

dietary fat and

fiber and relation-

ship of diet to can-

cer risk. Use of

smoking and

smokeless tobacco

and intent to use

increased with

grade level. Data

confirm need for

primary prevention

studies for this

population.

Families and

school personnel

reported positive

experiences with

the program.
The combined tobacco use prevention and dietary modification intervention appears to have had the most success in influencing youths away from tobacco use and toward a more healthy diet. 


\section{Appendix E \\ National Cancer Institute Research on Native Americans}

\begin{abstract}
Title: Epstein-Barr Virus Expression in Nasopharyngeal Carcinoma

PI: $\quad$ Nancy J. Raab-Traub, Ph.D.

Institution: University of North Carolina

Chapel Hill, North Carolina

Description: The goal of this research is to determine the role of Epstein-Barr Virus in the etiology of nasopharyngeal carcinoma, an epithelial malignancy that develops with high incidence in southern China, northern Africa, and among Eskimos.
\end{abstract}

Title: $\quad$ Epidemiological Studies of Diet and Cancer in Hawaii

PI: $\quad$ Laurence N. Kolonel, Ph.D.

Institution: University of Hawaii at Manoa Honolulu, Hawaii

Description: The overall objective of this project is to identify dietary risk and protective factors for cancer that will lead to the control of this disease.

\section{Title: Cancer Prevention Research Program \\ PI: $\quad$ Maureen M. Henderson, M.D. \\ Institution: Fred Hutchinson Cancer Research Center, Seattle, Washington}

Description: The goals of this research project are to develop (1) effective populationbased behavioral interventions for dietary change and smoking cessation, and (2) methodology for evaluating population-based interventions. The research also focuses on the inclusion of minority and hard-toreach groups in population-based research.
Title:

\section{Data-Based Cancer Intervention Research}

PI:

Institution:

John P. Middaugh, M.D.

Description: This project will serve as a model for the planning and evaluation of statewide cancer prevention and control interventions. The specific goals of this project are to (1) reduce Alaska's age-adjusted cancer mortality rate by 50 percent by the year 2000; (2) develop and institutionalize a comprehensive cancer prevention and control program in the Alaska Division of Public Health; and (3) ensure a mechanism to implement state-of-the-art techniques to translate research findings into public and private practice and to ensure that services are available to prevent and control cancer.

Title: $\quad$ Cancer Risk Reduction Among Native American Adolescents

PI: Michael S. Moncher, D.S.W.

Institution: Intersystems, Inc. New York, New York

Description: Building on Phase I results, this Phase II study will (1) develop software for delivering intervention to reduce cancer risks associated with tobacco use and dietary habits among Native American adolescents; (2) compare the effects of the software with conventional group intervention in a randomized clinical trial with a representative sample of Native American adolescents; and (3) analyze the costs of both modes of intervention delivery. 


\section{National Cancer Institute Research on Native Americans (continued)}

Data from Phase I established the feasibility of using interactive computer software in a curriculum of five 40 minute interactive lessons.

Title:

\section{Statistical Methods for Genetic Epidemiology of Cancer}

PI: $\quad$ Lue Ping Zhao, Ph.D

Institution: Fred Hutchinson Cancer Research Center, Seattle, Washington

Description: The broad objective of this project is to develop several statistical methods for genetic epidemiology of cancer involving a population-based cohort with pedigree information. These statistical methods will be applied to a population-based pedigree cohort of multiethnic groups in Hawaii. The results from the analysis will reveal patterns of familial aggregation of all major cancers. As a long-term objective, this project will provide leads to further research investigating roles of genes and environmental factors in cancer etiology.

Title: $\quad$ Epidemiology of Cervical Dysplasia in American Indian Women

PI: Melissa A. Schiff, M.D.

Institution: University of New Mexico Albuquerque, New Mexico

Description: To assess the risk factors for the development of moderate dysplasia, severe dysplasia, and carcinoma in situ in New Mexico's American Indian women, this project will conduct a case-control study at several Indian Health Service-affiliated outpatient clinics. The study design incorporates an integrated approach to investigate the relationship of numerous exposures in the development of highgrade dysplastic cervical lesions.
Title:

Race, Fatty Acid Exposure, and Risk of Prostate Cancer

PI:

Institution:

Paul A. Godley, M.D.

Description: This project will conduct a case-control study to test the theory that omega-3 fatty acids protect against prostate cancer. Cases will be 100 patients with pathologically confirmed, recently diagnosed prostate cancer recruited from the urology clinics of the University of North Carolina hospitals and the Durham Veterans Administration Medical Center. Controls will be 100 patients with benign prostatic hyperplasia who are free of prostate cancer on pathological examination of the prostate.

Title: Interactive VideoDisc Smoking Cessation Intervention

PI: John W. Noell, Ph.D.

Institution: Oregon Center for Applied Science Eugene, Oregon

Description: This project will produce an interactive, videodisc-based smoking cessation system for use in medical office settings.

Title:

p53 Breast Cancer Mutations in High- and Low-Risk Groups

PI: John S. Kovach

Institution: City of Hope National Medical Center, Duarte, California

Description: The objective of this project is to determine whether exposure to different exogenous mutagens is responsible for differences in the incidence and prognosis of breast cancer in ethnically and racially diverse populations. The approach is to compare the specific biochemical pattern of 


\section{National Cancer Institute Research on Native Americans (continued)}

mutation in the tumor suppressor gene, $\mathrm{p} 53$, in breast cancers from populations with low incidence of breast cancer, e.g., Japanese and Native Americans, to the pattern of mutation in populations with a high incidence of breast cancer, e.g., northern Europeans and North Americans.

Title:

PI:

Institution: North Dakota State University Fargo, North Dakota

Description: The purpose of this research is to increase the number of women in North Dakota who (1) obtain a firsttime mammography, and (2) continue to obtain screening at appropriate intervals. Communities in one-half of the State will receive a community intervention, whereas communities in the other part of the State will serve as on-treatment controls.

Title:

Dietary Intervention-Lung, Head, and Neck Cancer Patients

PI: $\quad$ Loic Le Marchand, Ph.D.

Institution: University of Hawaii at Manoa Honolulu, Hawaii

Description: This randomized intervention trial will test the hypothesis that a daily diet of five servings of green and yellow vegetables and four servings of fruits (particularly citrus fruits) will reduce the incidence of second primary malignancies among patients with stages I-II head and neck cancers or with stage I squamous cell carcinoma of the lung.
Title:

\section{American Indian Women's Talking Circle}

PI: $\quad$ Felicia Schanche Hodge, Dr.P.H.

Institution: University of California Berkeley, California

Description: This study will evaluate the efficacy of the "talking circle," a culturally appropriate psychosocial counseling intervention method, on the quality of life of American Indian women at risk for cervical cancer in northern California. Promoting healthy behavior and increased treatment compliance through the Native American oral tradition of storytelling is an intervention approach acceptable in our Indian communities.

Title: Breast Cancer Treatment Protocols for Hawaii Minorities

PI: $\quad$ Brian F. Issell, M.D.

Institution: University of Hawaii at Manoa Honolulu, Hawaii

Description: The purpose of this project is to promote breast cancer treatment among Hawaii's high-risk minority breast cancer patients. The project focuses on the development of novel treatment protocols which ensure access to state-of-the-art treatment and do not have the eligibility restrictions of national cooperative group protocols.

Title: Nutritional Circles To Reduce Cancer Risk

PI: $\quad$ Felicia Schanche Hodge, Dr.P.H.

Institution: University of California Berkeley, California

Description: This project will build on the findings from a previous study of dietary habits 


\section{National Cancer Institute Research on Native Americans (continued)}

of California Indians to design a culturally sensitive intervention utilizing the talking circle (nutritional circle). The intervention will focus on reinforcing positive food habits and modifying nutritionally detrimental ones to reduce the risk of cancer and other chronic diseases.

Title:

PI:

Institution:

Description: This project will test an intervention

\section{Yukon-Kuskokwim Women's Cancer Control Project}

Allen J. Dietrich, M.D.

Dartmouth College

Hanover, New Hampshire provided by the Yukon-Kuskokwim Health Corporation based on techniques of Continuous Quality Improvement (CQI) to increase indicated early detection and prevention of cancer services for Native Alaskan women. The intervention builds on the community health aide and village clinic system to better understand culturally appropriate needs and expectations of Native Alaskan women and to support active Native health provider participation and leadership in care delivery assessment and improvement. Specifically, the CQI approach will build on the culturally sensitive knowledge and skills of community health aides to develop a management process in each village clinic that empowers and activates both community women and health aides to achieve better early detection and prevention of cancer services.

Title:

Native Researchers Cancer Control Training Program

PI: $\quad$ Thomas M. Becker, M.D., Ph.D.

Institution: Oregon Health Science University Portland, Oregon

Description: This program will increase research capabilities of Native researchers to carry out well-designed cancer prevention and control studies within Native populations.

Title:

\section{Cancer Risk Assessment- Clinical and Molecular Tools}

PI:

Institution

Stephen N. Thibodeau, Ph.D.

Mayo Foundation

Rochester, Minnesota

Description: This project will (1) assess the association of family history with tumor microsatellite instability; (2) estimate the frequency of germline mutations and define the spectrum of mutations in the four genetic susceptibility loci among patients demonstrating tumor microsatellite instability and/or a positive family history; (3) develop and evaluate a questionnaire that is effective in identifying patients who are most likely to have a genetic predisposition for cancer; and (4) determine the frequency of tumor microsatellite instability in an Alaska Native population.

\section{Title: Breast Cancer Surveillance} Research in New Mexico

PI: $\quad$ Charles R. Key, M.D.

Institution: University of New Mexico Albuquerque, New Mexico

Description: This project will create a geographically defined and population-based resource in the State of New Mexico, a multiethnic and largely rural State. The core of the study is the expansion of the Albuquerque metropolitan area surveillance system (established as a pilot study by the New Mexico Tumor Registry in 1990) to include complete coverage of the State's rural areas. The resource will include complete ascertainment of screening mammograms, breast cancers, and benign breast tumors, and a popula- 


\section{National Cancer Institute Research on Native Americans (continued)}

tion-based survey of breast cancer risk factors and screening behaviors in screened and unscreened women.

Title:

\section{Novel Markers for \\ Prostate Cancer}

PI: $\quad$ C. Y. Young, Ph.D.

Institution: Mayo Foundation

Rochester, Minnesota

Description: This project will develop and evaluate new immunoassay and molecular detection systems for early detection and prognosis of prostate cancer.

Title: Breast Cancer Screening in a TriRacial Rural Population

PI: $\quad$ Electra D. Paskett, Ph.D.

Institution: Wake Forest University Winston-Salem, North Carolina

Description: The overall goal of this project is to increase early detection of breast cancer by increasing the proportion of low-income rural women, age 50 and older, who receive clinical breast exams and mammograms at appropriate intervals and return for followup care when necessary.
Title:

\section{Hawaii Family Registry of Colon Cancer}

PI: $\quad$ Ioic Le Marchand, Ph.D.

Institution: University of Hawaii at Manoa

Honolulu, Hawaii

Description: The aims of this project are to (1) establish a family registry and biorepository for high-risk families for colon cancer in Hawaii and to contribute data and specimens for about 130 new intermediate and high-risk families for colorectal cancer, and (2) develop and pilot test protocols using the cooperative Family Registry.

\section{Title: Bupropion Treatment for} Youth Smoking Cessation

PI: $\quad$ Scott J. Leischow, Ph.1).

Institution: University of Arizona Tucson, Arizona

Description: The proposed study is a randomized, double-blind, placebo-controlled clinical trial designed to assess (1) which bupropion dose, if any, increases ahstinence rates over placebo, and (2) which factors, such as medication adherence, nicotine dependence, and motivation to quit, affect treatment outcome. 


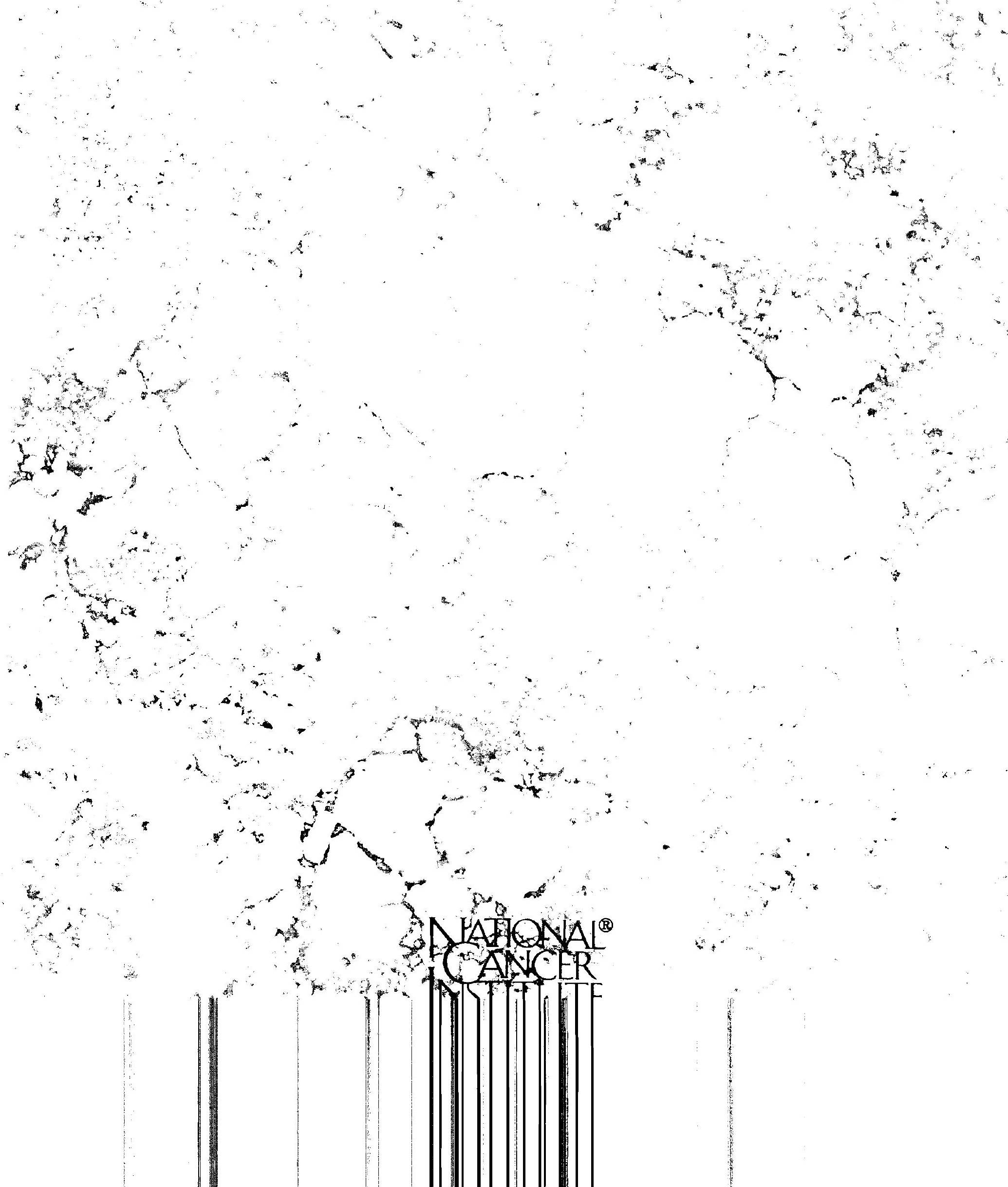

